University of Tennessee Health Science Center

UTHSC Digital Commons

\title{
The Role of Multi-Drug Resistance Associated Protein 4 and P- glycoprotein in Resistance of Neuroblastoma to Topotecan and Irinotecan
}

\author{
Patricia Kellie Turner \\ University of Tennessee Health Science Center
}

Follow this and additional works at: https://dc.uthsc.edu/dissertations

Part of the Amino Acids, Peptides, and Proteins Commons, Medicinal and Pharmaceutical Chemistry Commons, Neoplasms Commons, Pharmaceutical Preparations Commons, and the Pharmaceutics and Drug Design Commons

\section{Recommended Citation}

Turner, Patricia Kellie, "The Role of Multi-Drug Resistance Associated Protein 4 and P-glycoprotein in Resistance of Neuroblastoma to Topotecan and Irinotecan" (2007). Theses and Dissertations (ETD). Paper 269. http://dx.doi.org/10.21007/etd.cghs.2007.0325. 


\title{
The Role of Multi-Drug Resistance Associated Protein 4 and P-glycoprotein in Resistance of Neuroblastoma to Topotecan and Irinotecan
}

\begin{abstract}
High-risk neuroblastoma presents a significant therapeutic challenge because the 5-year survival rate remains less than $30 \%$ despite the use of surgery, multi-agent chemotherapy, radiation, and autologous bone marrow transplant. Novel therapeutic modalities are under development. The camptothecin analogs topotecan and irinotecan have been identified as successful cytotoxic agents. For topotecan, pharmacokinetically guided dosing to achieve a systemic exposure associated with preclinical anti-tumor activity in neuroblastoma xenograft models is feasible and has elicited favorable responses in children with high-risk neuroblastoma. However, some children with high-risk disease did not respond to the putatively effective topotecan systemic exposure. These children represent a subset of the disease intrinsically resistant to topotecan. Furthermore, mRNA expression of the adenosine triphosphate (ATP)binding cassette (ABC) transporters P-glycoprotein (Pgp) and multidrug resistance associated protein 1 (MRP1), which efflux many drugs used in neuroblastoma therapy, has been implicated in poor outcome in neuroblastoma. Therefore, the purpose of our studies was to determine the role of $A B C$ transport protein expression in neuroblastoma resistance to the camptothecin analogs topotecan and irinotecan.
\end{abstract}

Initially studies focused on determining the expression of $A B C$ transporters for which the camptothecin analogs are substrates in neuroblastoma cell lines. By western blot analysis we demonstrated MRP4 and Pgp expression in neuroblastoma cell lines relatively resistant to topotecan (e.g., NB1691), but not in cell lines sensitive to topotecan (e.g., NB1643). In contrast, MRP1, MRP2, and breast cancer resistance protein (BCRP) expression did not discriminate between sensitive and resistant cell lines. To determine the functional contribution of both MRP4 and Pgp in neuroblastoma, we used RNA interference (RNAi) to silence MRP4 and Pgp expression in NB1691. Long term, stable expression of retroviral vector mediated short hairpin RNA (shRNA) reduced MRP4 and Pgp expression. Isogenic cell lines with reduced expression of MRP4 and Pgp exhibited an increase in sensitivity to both topotecan and SN-38, the active moiety of the prodrug irinotecan. In addition, we overexpressed MRP4 in NB1643, which resulted in increased topotecan resistance.

The NB1691 cell lines with reduced MRP4 expression were subsequently transplanted as xenografts into severe combined immunodeficiency (SCID) mice to determine the effect of MRP4 expression on the in vivo response to topotecan. Unexpectedly, MRP4 silencing did not persist in vivo, and none of the xenograft models responded to topotecan. However, MRP4 expression was associated with failure to respond to topotecan, supporting the hypothesis that MRP4 mediates resistance to topotecan.

Finally, we determined the $A B C$ transporter expression profile in primary tumor specimens from patients with high-risk neuroblastoma who were treated with pharmacokinetically guided topotecan. Of the 14 specimens studied, MRP4 was expressed in 2 samples, and Pgp was expressed in 4 samples. BCRP was not expressed in any of the neuroblastoma cell lines in vitro, but immunohistochemical analysis demonstrated BCRP expression in nine primary neuroblastoma samples. Although we predicted that MRP4 and/or Pgp expression would be associated with failure to respond to topotecan, results of immunohistochemical analysis did not demonstrate such an association.

The results of the in vitro studies demonstrate that MRP4 and Pgp confer resistance to topotecan and $\mathrm{SN}-38$. In the xenograft studies, MRP4 expression was associated with failure to respond to topotecan. However, this phenotype was not recapitulated in children treated with topotecan. These results may be confounded by small sample size and timing of sample acquisition. Further investigation of the role of $A B C$ transporters in children with neuroblastoma who receive either topotecan or irinotecan may be warranted. In addition to the camptothecin analogs, patients will receive other drugs effluxed by the $A B C$ 
transporters (e.g., doxorubicin, vincristine, etoposide, cyclophosphamide). Therefore, analyzing ABC transporter expression by immunohistochemistry in diagnostic tumor specimens may help to select agents not subject to efflux by $A B C$ transporters expressed in the tumor. However, eliminating drugs effluxed by $A B C$ transporters from the treatment regimen creates a potential gap in therapy and may reduce drug intensity. Therefore, further rational design and development of drugs that evade $A B C$ transporter-mediated efflux, and potentially other resistance mechanisms in neuroblastoma, is also warranted.

\section{Document Type}

Dissertation

Degree Name

Doctor of Philosophy $(\mathrm{PhD})$

\section{Program}

Pharmaceutical Sciences

\section{Research Advisor}

Clinton Stewart, Pharm.D.

\section{Keywords}

Neuroblastoma, topotecan, irinotecan, ABC transporter, multidrug resistance, MRP4, Pgp, RNAi, shRNA, siRNA, immunohistochemistry

\section{Subject Categories}

Amino Acids, Peptides, and Proteins | Chemicals and Drugs | Diseases | Medicinal and Pharmaceutical Chemistry | Medicine and Health Sciences | Neoplasms | Pharmaceutical Preparations | Pharmaceutics and Drug Design | Pharmacy and Pharmaceutical Sciences 


\title{
THE ROLE OF MULTI-DRUG RESISTANCE ASSOCIATED PROTEIN 4 AND P-GLYCOPROTEIN IN RESISTANCE OF NEUROBLASTOMA TO TOPOTECAN AND IRINOTECAN
}

\author{
A Dissertation \\ Presented for \\ The Graduate Studies Council \\ The University of Tennessee \\ Health Science Center
}

In Partial Fulfillment

Of the Requirements for the Degree

Doctor of Philosophy

From The University of Tennessee

By

Patricia Kellie Turner

December 2007 


\section{Dedication}

I dedicate this work to my parents Patricia and Doug Turner, my sister Kim

Turner, and my grandmother Helen Turner. Their constant support, encouragement and unconditional love have always inspired me in all of my endeavors. 


\section{Acknowledgements}

I am indebted to my mother, father, sister, and grandmother for their unconditional love and support. I am eternally grateful to my mentor and role model Dr. Clinton Stewart for his guidance and support. I will always appreciate his patience, the numerous opportunities he has provided, and all of the time he has spent teaching me. Other important teachers in my life whose time and guidance I truly appreciate are the members of my graduate committee: Drs. Mary Danks, Bernd Meibohm, Victor Santana, and John Schuetz. I thank Suzan Hanna, my "Mom" in the lab, for everything that she taught me and for her constant encouragement. I also thank many others who have helped me along the way, including Brad Johnston, Dr. Mark Leggas, Chuck Fraga, Dr. Stacy Throm, Dr. Jiaowang Dong, Dr. Lisa Iacono, Dr. Burgess Freeman, Dr. Bill Zamboni, Dr. Yanli Zhuang, Jun Shen, Geeta Nair, Dr. Feng Bai, Nik Hagedorn, Elaine Hubbard, Dr. Carl Panetta, Dr. Peter Houghton, Chris Morton, Klo Spelshouse, Peter Barta, Seth Dixon, Rebecca Bush, Jessie Morgan, Mark Wilkinson, Dr. Partha Krishnamurthy, Dr. Kelly Mercer, and Dr. Charis Marston. 


\begin{abstract}
High-risk neuroblastoma presents a significant therapeutic challenge because the 5-year survival rate remains less than $30 \%$ despite the use of surgery, multi-agent chemotherapy, radiation, and autologous bone marrow transplant. Novel therapeutic modalities are under development. The camptothecin analogs topotecan and irinotecan have been identified as successful cytotoxic agents. For topotecan, pharmacokinetically guided dosing to achieve a systemic exposure associated with preclinical anti-tumor activity in neuroblastoma xenograft models is feasible and has elicited favorable responses in children with high-risk neuroblastoma. However, some children with highrisk disease did not respond to the putatively effective topotecan systemic exposure. These children represent a subset of the disease intrinsically resistant to topotecan. Furthermore, mRNA expression of the adenosine triphosphate (ATP)-binding cassette $(\mathrm{ABC})$ transporters $\mathrm{P}$-glycoprotein $(\mathrm{Pgp})$ and multidrug resistance associated protein 1 (MRP1), which efflux many drugs used in neuroblastoma therapy, has been implicated in poor outcome in neuroblastoma. Therefore, the purpose of our studies was to determine the role of $\mathrm{ABC}$ transport protein expression in neuroblastoma resistance to the camptothecin analogs topotecan and irinotecan.

Initially studies focused on determining the expression of $\mathrm{ABC}$ transporters for which the camptothecin analogs are substrates in neuroblastoma cell lines. By western blot analysis we demonstrated MRP4 and Pgp expression in neuroblastoma cell lines relatively resistant to topotecan (e.g., NB1691), but not in cell lines sensitive to topotecan (e.g., NB1643). In contrast, MRP1, MRP2, and breast cancer resistance protein (BCRP) expression did not discriminate between sensitive and resistant cell lines. To determine
\end{abstract}


the functional contribution of both MRP4 and Pgp in neuroblastoma, we used RNA interference (RNAi) to silence MRP4 and Pgp expression in NB1691. Long term, stable expression of retroviral vector mediated short hairpin RNA (shRNA) reduced MRP4 and Pgp expression. Isogenic cell lines with reduced expression of MRP4 and Pgp exhibited an increase in sensitivity to both topotecan and $\mathrm{SN}-38$, the active moiety of the prodrug irinotecan. In addition, we overexpressed MRP4 in NB1643, which resulted in increased topotecan resistance.

The NB1691 cell lines with reduced MRP4 expression were subsequently transplanted as xenografts into severe combined immunodeficiency (SCID) mice to determine the effect of MRP4 expression on the in vivo response to topotecan. Unexpectedly, MRP4 silencing did not persist in vivo, and none of the xenograft models responded to topotecan. However, MRP4 expression was associated with failure to respond to topotecan, supporting the hypothesis that MRP4 mediates resistance to topotecan.

Finally, we determined the $\mathrm{ABC}$ transporter expression profile in primary tumor specimens from patients with high-risk neuroblastoma who were treated with pharmacokinetically guided topotecan. Of the 14 specimens studied, MRP4 was expressed in 2 samples, and Pgp was expressed in 4 samples. BCRP was not expressed in any of the neuroblastoma cell lines in vitro, but immunohistochemical analysis demonstrated BCRP expression in nine primary neuroblastoma samples. Although we predicted that MRP4 and/or Pgp expression would be associated with failure to respond to topotecan, results of immunohistochemical analysis did not demonstrate such an association. 
The results of the in vitro studies demonstrate that MRP4 and Pgp confer resistance to topotecan and $\mathrm{SN}-38$. In the xenograft studies, MRP4 expression was associated with failure to respond to topotecan. However, this phenotype was not recapitulated in children treated with topotecan. These results may be confounded by small sample size and timing of sample acquisition. Further investigation of the role of $\mathrm{ABC}$ transporters in children with neuroblastoma who receive either topotecan or irinotecan may be warranted. In addition to the camptothecin analogs, patients will receive other drugs effluxed by the $\mathrm{ABC}$ transporters (e.g., doxorubicin, vincristine, etoposide, and cyclophosphamide). Therefore, analyzing $\mathrm{ABC}$ transporter expression by immunohistochemistry in diagnostic tumor specimens may help to select agents not subject to efflux by $\mathrm{ABC}$ transporters expressed in the tumor. However, eliminating drugs effluxed by $\mathrm{ABC}$ transporters from the treatment regimen creates a potential gap in therapy and may reduce drug intensity. Therefore, further rational design and development of drugs that evade $\mathrm{ABC}$ transporter-mediated efflux, and potentially other resistance mechanisms in neuroblastoma, is also warranted. 


\section{Table of Contents}

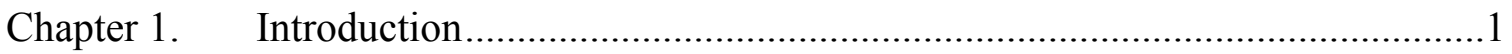

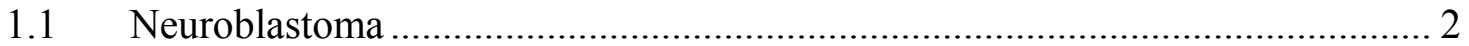

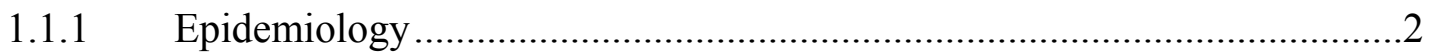

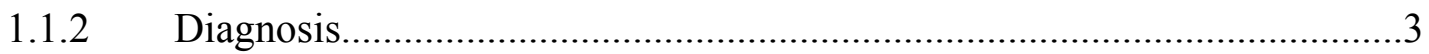

1.1.3 Prognostic indicators and risk adapted therapy ………..............................

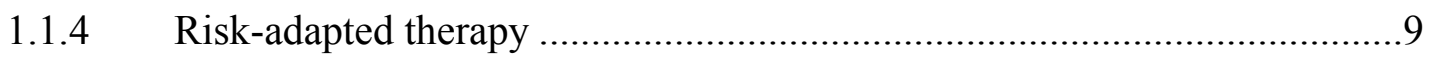

1.2 Camptothecin analogs are topoisomerase I interactive agents ........................ 13

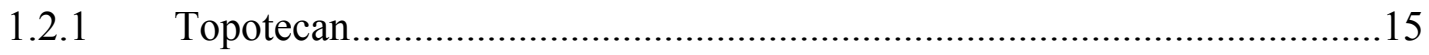

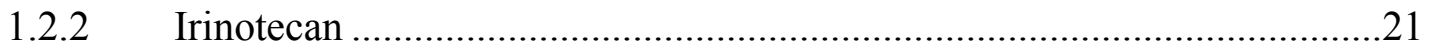

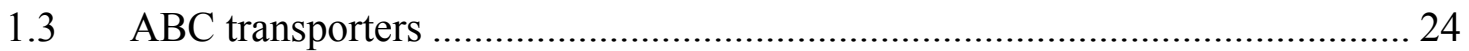

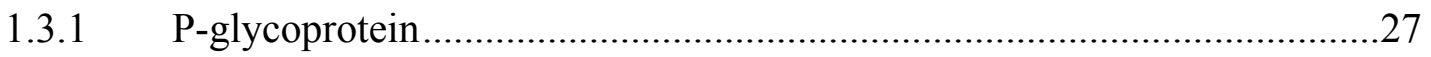

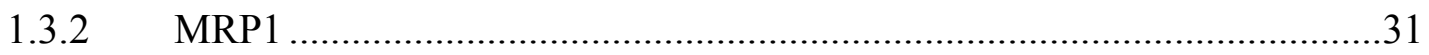

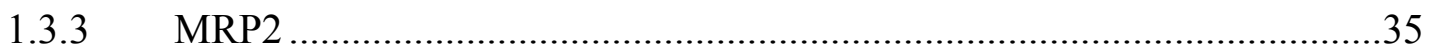

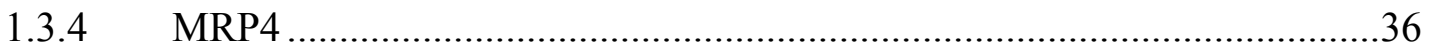

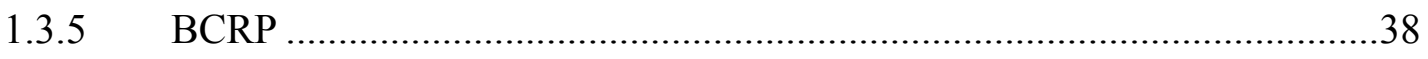

1.3.6 Modifying ABC transporter function..........................................................41

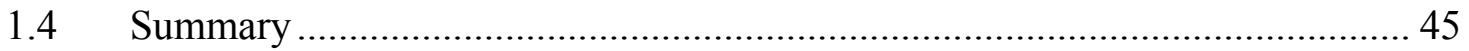

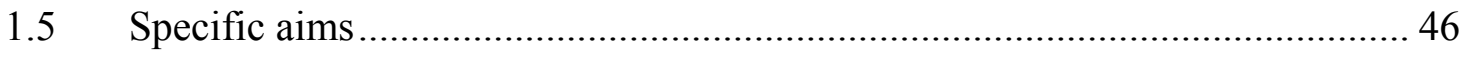

1.5.1 To determine the contribution of $\mathrm{ABC}$ transporters to topotecan and irinotecan sensitivity in neuroblastoma cell lines ................................46

1.5.2 To determine the role of MRP4 in neuroblastoma xenograft response to topotecan

1.5.3 To determine the role of $\mathrm{ABC}$ transporter expression in response to topotecan in children with high-risk neuroblastoma. 
Chapter 2. MRP4 and P-glycoprotein confer resistance to topotecan in neuroblastoma

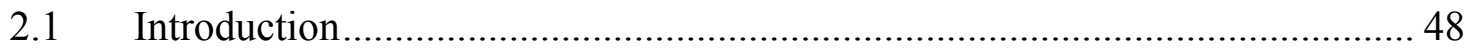

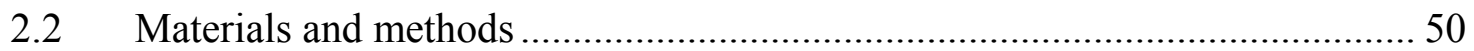

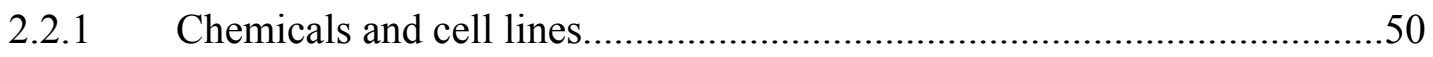

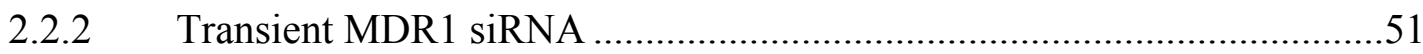

2.2.3 Stable RNAi by cloning MRP4 or MDR1 shRNA into MSCVGFP .........51

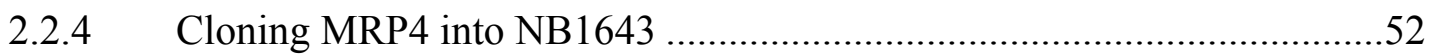

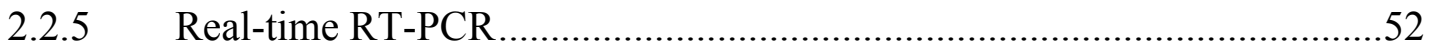

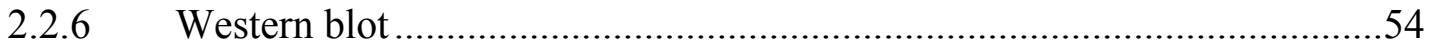

2.2.7 Intracellular topotecan, PMEA, rhodamine 123, and mitoxantrone

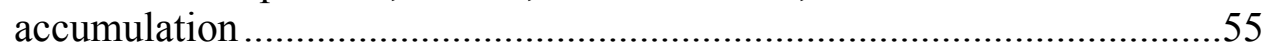

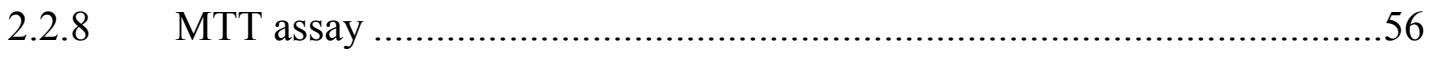

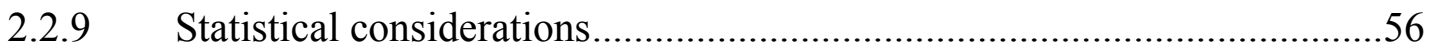

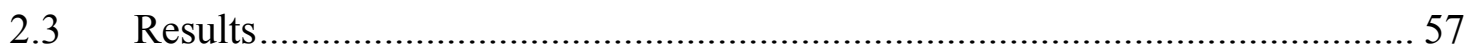

2.3.1 Functional MRP4 and Pgp expression in neuroblastoma cell lines resistant to camptothecin analogs ...........................................................57

2.3.2 Functional MRP4 knockdown with stably expressed shRNA ....................60

2.3.3 Functional MRP4 overexpression in NB1643 ..........................................66

2.3.4 Functional Pgp knockdown with stably expressed shRNA …………….....70

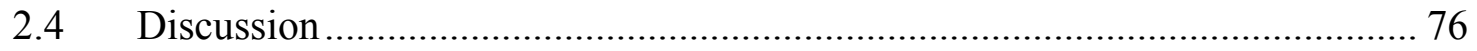

Chapter 3. Effect of MRP4 shRNA on NB1691 xenograft response to

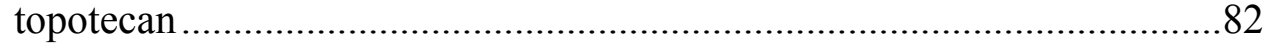

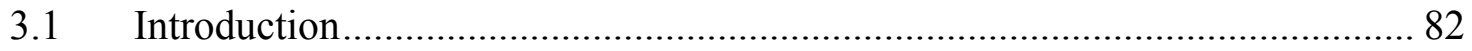

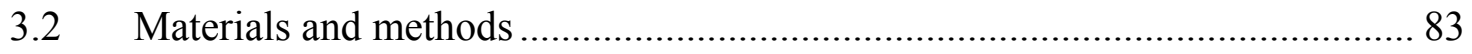

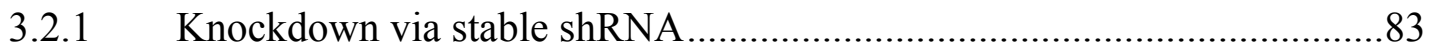

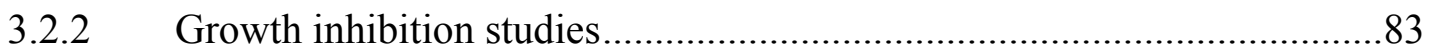




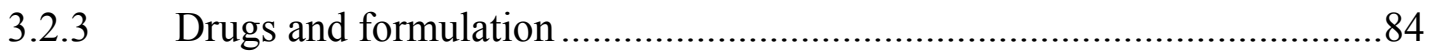

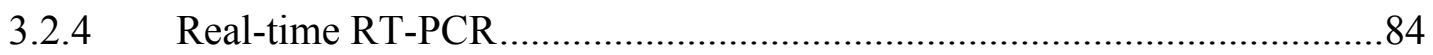

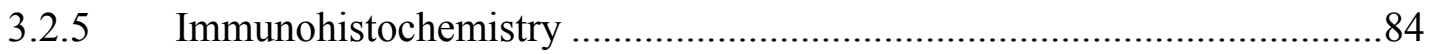

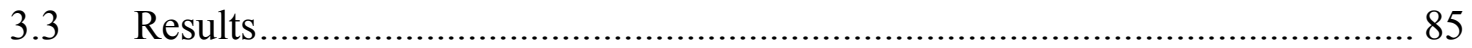

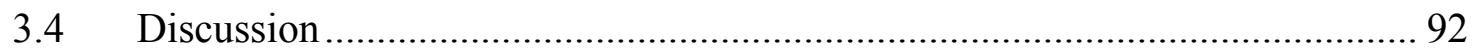

Chapter 4. $\mathrm{ABC}$ transporter expression in neuroblastoma biopsy specimens from children treated with pharmacokinetically guided topotecan.............96

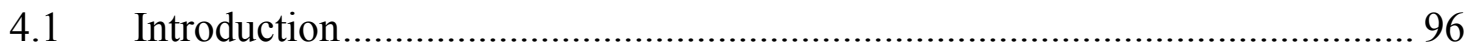

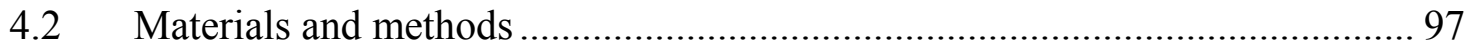

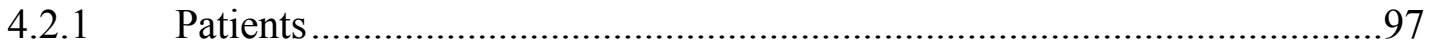

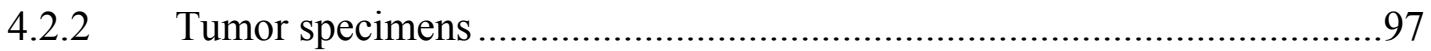

4.2.3 Definitions of response to pharmacokinetically guided topotecan ............97

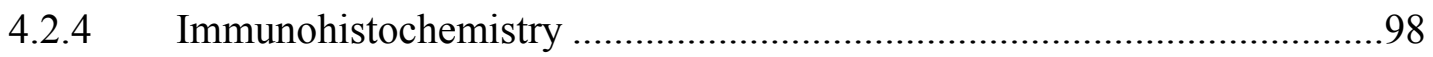

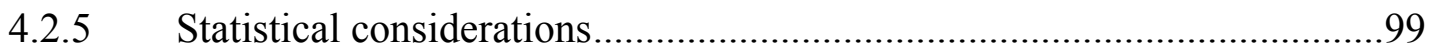

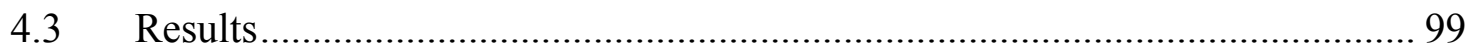

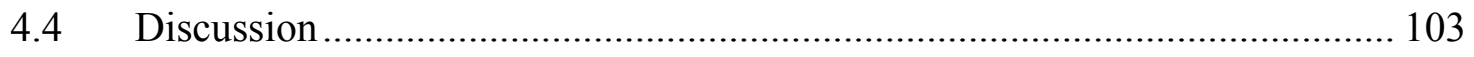

Chapter 5. Summary and future directions .............................................................106

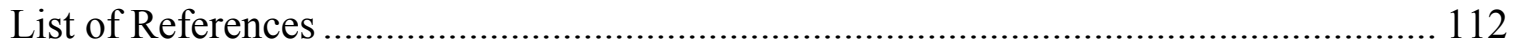

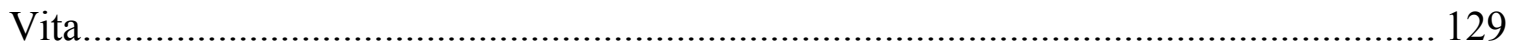




\section{List of Tables}

Table $1.1 \quad$ Neuroblastoma risk group stratification.............................................

Table 1.2 International staging system for neuroblastoma. ..................................6

Table 1.3 Topotecan clinical trials in children with recurrent or refractory solid tumors including neuroblastoma..................................................17

Table $1.4 \quad$ Clinical trials of topotecan pharmacokinetically guided dosing in children with solid tumors including neuroblastoma. 19

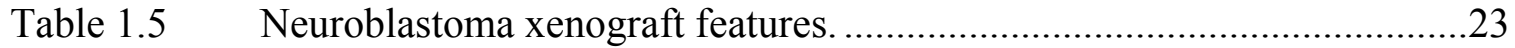

Table 1.6 Irinotecan phase I clinical trials in children with recurrent or refractory solid tumors including neuroblastoma. ...............................25

Table 1.7 Partial list of endogenous and xenobiotic MRP1 substrates......................32

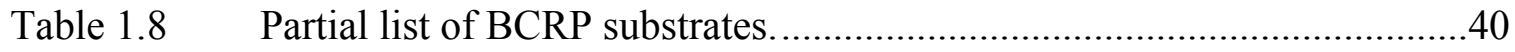

Table 1.9 Chemical modulators of ABC transporters........................................42

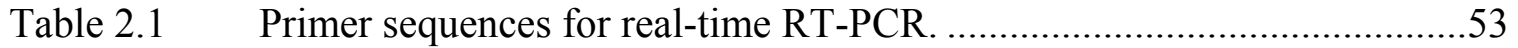

Table 4.1 ABC transporter expression in neuroblastoma specimens and response to pharmacokinetically guided topotecan. 


\section{List of Figures}

Figure 1.1 Camptothecin analog chemical structure.

Figure 2.1 MRP4 and Pgp expression in topotecan resistant neuroblastoma cell lines. .58

Figure 2.2 Accumulation of the MRP4 and Pgp substrates PMEA and rhodamine 123 in neuroblastoma cell lines.

Figure $2.3 \quad{ }^{3}$ H-Topotecan sensitivity and accumulation in neuroblastoma cell lines.

Figure 2.4 Immunoblot analysis of MRP4 expression in NB1691 cells transduced with MRP4 shRNA.

Figure 2.5 Real-time RT-PCR analysis in NB1691 MRP4 shRNA clones.

Figure 2.6 PMEA intracellular accumulation in NB1691 MRP4 shRNA clones. .64

Figure 2.7 Topotecan sensitivity in NB1691 MRP4 shRNA clones. .65

Figure 2.8 SN38 sensitivity in NB1691 MRP4 shRNA clones.

Figure 2.9 Immunoblot analysis of MRP4 expression in NB1643 cells transduced with MSCV MRP4 IRES GFP. .68

Figure 2.10 Intracellular PMEA accumulation in NB1643 cells overexpressing MRP4.

Figure 2.11 Topotecan sensitivity in NB1643 cells overexpressing MRP4. . .71

Figure 2.12 SN-38 sensitivity in NB1643 cells overexpressing MRP4. .72

Figure 2.13 Real-time RT-PCR analysis of in NB1691 MDR1 shRNA clones. .73

Figure 2.14 Intracellular mitoxantrone accumulation in NB1691 MDR1 shRNA clones.

Figure 2.15 Intracellular ${ }^{14} \mathrm{C}$-topotecan accumulation in NB1691 MDR1 shRNA clones. .75

Figure 2.16 Topotecan sensitivity in NB1691 MDR1 shRNA clones .77

Figure 2.17 SN-38 sensitivity in NB1691 MDR1 shRNA clones. .78 
Figure 3.1 NB1691 MRP4 shRNA xenograft response to i.v. topotecan 1

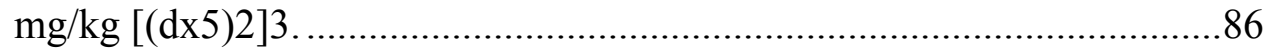

Figure 3.2 NB1691 MRP4 shRNA xenograft response to i.v. topotecan 2

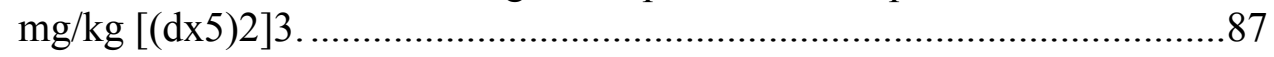

Figure 3.3 MRP4 mRNA expression in NB1691 MRP4 shRNA xenografts. .............89

Figure 3.4 MRP4 is expressed in NB1691 MRP4 shRNA xenografts.......................90

Figure 3.5 MDR1 mRNA expression in NB1691 MRP4 shRNA xenografts.............91

Figure 4.1 $\mathrm{ABC}$ transporter expression in human kidney controls...........................100

Figure 4.2 $\quad \mathrm{ABC}$ transporter expression in neuroblastoma tissue obtained during primary tumor resection. 


\section{List of Abbreviations}

$\mathrm{ABC}$

ATP-binding cassette

$\mathrm{ABCB} 1$ gene member 1 of ABC subfamily B (alias MDR1)

ABCC1-4 gene members $1-4$ of $\mathrm{ABC}$ subfamily $\mathrm{C}$

$\mathrm{ABCG} 2$ gene member 2 of $\mathrm{ABC}$ subfamily $\mathrm{G}$ (BCRP gene)

$\mathrm{ABCP}$ placenta resistance protein (alias BCRP)

AraC cytarabine

ATP adenosine triphosphate

AUC area under the concentration-time curve

BCRP breast cancer resistance protein

Bcrp breast cancer resistance protein, mouse cAMP cyclic adenosine monophosphate

CDDP cisplatin

cGMP cyclic guanosine monophosphate

CI continuous infusion

cMOAT canalicular multispecific organic anion transporter

COG Children's Oncology Group

CR complete response $\mathrm{C}_{\mathrm{t}}$ cycle threshold

CTX cyclophosphamide

Dauno daunorubicin

DLT dose limiting toxicity DNA deoxyribonucleic acid 
Dox doxorubicin

dsRNA double stranded RNA

$\mathrm{E}_{2} 17 \beta \mathrm{G}$ estradiol-17- $\beta$-D-glucuronide

EST expressed sequence tag

FACS fluorescence activated cell sorter

FDA Food and Drug Administration

GAPDH glyceraldehydes-3-phosphate dehydrogenase

GFP. green fluorescent protein

G-CSF granulocyte-colony stimulating factor (filgrastim)

i.p. intraperitoneal

INSS International Neuroblastoma Staging System IRES internal ribosomal entry site i.v. intravenous

$\mathrm{kDa}$ kiloDalton

MDR1 gene for multidrug resistance protein 1

Mdr1a/1b multidrug resistance protein $1 \mathrm{a}$ and $1 \mathrm{~b}$, mouse MEF murine embryonic fibroblast

6-MP 6-mercaptopurine mRNA messenger RNA

Mrp1 multidrug resistance associated protein, mouse MRP1-4 multidrug resistance associated protein 1-4 Mrp4 multidrug resistance associated protein 4 , mouse MSCV murine stem cell virus 
MTD maximum tolerated dose

MXR mitoxantrone resistance protein (alias BCRP)

NB neuroblastoma

NBD nucleotide binding domain

OR objective response

p.o. per os (oral)

PCR polymerase chain reaction $\mathrm{PGE}_{1-2}$ prostaglandin E 1-2

Pgp P-glycoprotein PMEA 9-(2-phosphonylymethoxyethyl) adenine POG Pediatric Oncology Group

PR partial response RNA ribonucleic acid RNAi RNA interference RT-PCR reverse transcriptase PCR $\mathrm{SD}$ stable disease SDS-PAGE sodium dodecyl sulfate polyacrylamide gel electrophoresis shRNA short hairpin RNA siRNA small interfering RNA

$\mathrm{SN}-38$ irinotecan metabolite SNP single nucleotide polymorphism 6-TG 6-thioguanine TMD transmembrane domain 
VM-26 teniposide VP-16 etoposide 


\section{Chapter 1. Introduction}

Neuroblastoma is the most common extracranial solid tumor of childhood, accounting for $7-10 \%$ of all childhood malignancies $(1,2)$. Most patients present with metastatic disease and have a poor prognosis $(2,3)$. Therefore, improving therapy for children with neuroblastoma is a major focus of ongoing preclinical and clinical research. Results of work in one such area, overcoming multi-drug resistance, suggest that it may be one of the many barriers to improving survival in children with this disease. The molecular basis of multi-drug resistance in neuroblastoma is likely to be multifactorial, caused by alterations in DNA repair pathways (4), increased intracellular detoxification/ inactivation of drugs $(5,6)$, alterations in cell survival pathways $(7,8)$, decreased binding of drugs to the intended target (9), or increased efflux of chemotherapeutic agents from cells.

Available data suggest that in neuroblastoma cells increased efflux may be a determinant of resistance to chemotherapy. In solid tumor cells, drug efflux can be mediated by members of the $\mathrm{ABC}$ transport protein superfamily. P-glycoprotein (Pgp) and multi-drug resistance associated protein 1 (MRP1), both members of the $\mathrm{ABC}$ transporter superfamily, are expressed in tumor specimens from children with neuroblastoma $(10,11,12)$. High Pgp and/ or MRP1 expression in neuroblastoma is associated with poor prognosis. Also associated with poor prognosis is MYCN, a transcription factor often amplified in neuroblastoma (13). The observation that MYCN regulates the expression of several $\mathrm{ABC}$ transporters suggests two independent 
prognostic indicators for poor outcome: $\mathrm{MYCN}$ amplification and $\mathrm{ABC}$ transporter expression (14).

Several drugs used in neuroblastoma therapy, including doxorubicin, vincristine, and etoposide $(15,16)$, are $\mathrm{ABC}$ transporter substrates. The camptothecin analogs topotecan and irinotecan are emerging as useful components of neuroblastoma therapy $(15,17)$ and are also substrates for ABC transporters $(18)$. Therefore, the major focus of this dissertation will be to assess the role of $\mathrm{ABC}$ transporter expression in resistance to the cytotoxicity of topotecan and irinotecan in vitro, in vivo, and in children with neuroblastoma. Expression of $\mathrm{ABC}$ transporters for which these camptothecin analogs are substrates will be characterized in neuroblastoma cell lines demonstrating a spectrum of sensitivity to these compounds. Molecular techniques including exogenous overexpression and RNA interference (RNAi) will be used to evaluate the functional contribution of individual transporters to drug resistance in vitro by quantitating camptothecin analog sensitivity in isogenic cell lines. In addition, xenografts in mice will be generated using these isogenic cell lines to assess the response to camptothecin analogs. Lastly, primary tumor specimens from patients with high-risk neuroblastoma will be analyzed to identify potential associations between clinical response to topotecan and $\mathrm{ABC}$ transporter expression.

\section{$1.1 \quad$ Neuroblastoma}

\subsubsection{Epidemiology}

Neuroblastoma is the most common cancer in children younger than one year (19). Furthermore, neuroblastoma is the most common extracranial solid tumor of 
childhood. Neuroblastoma can arise from any tissue of sympathetic origin, but the adrenal gland is the most common primary site. In the United States the annual neuroblastoma incidence among children less than 15 years of age is 9.7 cases per million (20). Neuroblastoma occurs at similar rates in both males and females, but a slightly higher predominance is reported in whites than in blacks (10.2 versus 7.8 cases per million, respectively). Most commonly neuroblastoma occurs in children younger than two years. The median age at diagnosis is 22 months (21). Between 1985 and 1988 the overall 5-year survival for children with neuroblastoma was 58\% (1). However, only $34 \%$ of children diagnosed with stage 4 neuroblastoma are long-term survivors (22). The biological mechanisms that contribute to the poor survival in children with advanced stage neuroblastoma are a subject of intense research. $\mathrm{ABC}$ transporter mediated efflux of chemotherapeutic agents from tumor cells is among the many potential causes of poor survival in children with neuroblastoma. Therefore, the major focus of this dissertation will be on the role of $\mathrm{ABC}$ transporters in high-risk neuroblastoma, in particular, the role of these proteins as determinants of sensitivity or resistance to the camptothecin analogs topotecan and irinotecan.

\subsubsection{Diagnosis}

Patients with neuroblastoma can present with abdominal discomfort, fullness, or pain (23). Hypertension, sweating, flushing, and tachycardia may also be present, depending on tumoral catecholamine secretion. Before 1988 no uniform criteria for neuroblastoma diagnosis, staging, and response evaluation existed, making it difficult to compare clinical studies of neuroblastoma(24). From 1988-1993 the major pediatric oncology groups from the United States, Europe, and Japan proposed consensus criteria 
for neuroblastoma diagnosis, staging, and response evaluation (25). Tumor stage and other prognostic indicators (e.g., MYCN status, DNA ploidy, Shimada Histology) are assessed in order to stratify patients into risk groups which determine the course of therapy.

\subsubsection{Prognostic indicators and risk adapted therapy}

\subsubsection{Neuroblastoma disease stage as a prognostic indicator}

Therapeutic modalities and treatment intensity are determined based upon a child's risk for a poor outcome (i.e., low, intermediate, and high-risk disease). Our study focuses on high-risk neuroblastoma. Factors used to stratify neuroblastoma patients into low, intermediate, or high-risk neuroblastoma (Table 1.1) include disease stage according to the international staging system for neuroblastoma (INSS), a patient's age, MYCN gene copy number, Shimada histology, and DNA index (2). The patient's age at diagnosis and INSS stage are the two most important prognostic variables in childhood neuroblastoma. The current standardized INSS defines six possible stages (Table 1.2) of childhood neuroblastoma based upon tumor confinement, resection, metastasis to lymph nodes, bone marrow, liver, or other sites (2). Children with advanced neuroblastoma (i.e., stage 3 or 4) have a poor prognosis.

\subsubsection{Age at neuroblastoma diagnosis as a prognostic indicator}

The correlation between young age at diagnosis (i.e., younger than 12-18 months) and good prognosis is well documented (26). Recent evidence suggests that children younger than 18 months have a more favorable prognosis than older children diagnosed 
Table 1.1 Neuroblastoma risk group stratification.

\begin{tabular}{llllll}
\hline $\begin{array}{l}\text { INSS } \\
\text { Stage }\end{array}$ & $\begin{array}{l}\text { Age } \\
\text { (Years) }\end{array}$ & MYCN Status & $\begin{array}{l}\text { Shimada } \\
\text { Histology }\end{array}$ & DNA Index & Risk Group \\
\hline 1 & $0-21$ & Any & Any & Any & Low \\
$2 \mathrm{~A} / 2 \mathrm{~B}$ & $<1$ & Any & Any & Any & Low \\
& $\geq 1-21$ & Non-amplified & Any & NA & Low \\
& $\geq 1-21$ & Amplified & Favorable & NA & Low \\
& $\geq 1-21$ & Amplified & Unfavorable & NA & High \\
3 & $<1$ & Non-amplified & Any & Any & Intermediate \\
& $<1$ & Amplified & Any & Any & High \\
& $\geq 1-21$ & Non-amplified & Favorable & NA & Intermediate \\
& $\geq 1-21$ & Non-amplified & Unfavorable & NA & High \\
& $\geq 1-21$ & Amplified & Any & NA & High \\
& $<1$ & Non-amplified & Any & Any & Intermediate \\
& $<1$ & Amplified & Any & Any & High \\
& $\geq 1-21$ & Any & Any & NA & High \\
& $<1$ & Non-amplified & Favorable & $>1$ & Low \\
& $<1$ & Non-amplified & Any & $=1$ & Intermediate \\
& $<1$ & Non-amplified & Unfavorable & Any & Intermediate \\
& $<1$ & Amplified & Any & Any & High \\
\hline
\end{tabular}

Source: Castleberry, RP. Neuroblastoma, Eur J Cancer, 33: 1430-1437, 1997. 


\section{Table 1.2 International staging system for neuroblastoma.}

\begin{tabular}{ll}
\hline Stage & Description \\
\hline Stage 1 & $\begin{array}{l}\text { Localized tumor confined to area of origin; complete gross resection, } \pm \\
\text { microscopic residual disease; ipsilateral and contralateral lymph nodes } \\
\text { are tumor negative }\end{array}$ \\
Stage 2A & $\begin{array}{l}\text { Unilateral with incomplete gross resection; ipsilateral and contralateral } \\
\text { lymph nodes tumor negative }\end{array}$ \\
Stage 2B & $\begin{array}{l}\text { Unilateral with complete or incomplete gross resection; ipsilateral lymph } \\
\text { node tumor positive; contralateral lymph node tumor negative } \\
\text { Tumor infiltrates across midline } \pm \text { regional lymph node involvement; or } \\
\text { unilateral tumor with contralateral node involvement; or midline tumor }\end{array}$ \\
Stage 3 & $\begin{array}{l}\text { with bilateral lymph node involvement } \\
\text { Tumor dissemination to distant lymph nodes, bone marrow, liver, or } \\
\text { other organ except as in } 4 \mathrm{~S}\end{array}$ \\
Stage 4 & $\begin{array}{l}\text { Localized primary tumor as for Stage 1 or 2 with dissemination to liver, } \\
\text { skin, or bone marrow with }<10 \% \text { nucleated marrow cells being tumor } \\
\text { cells }\end{array}$ \\
\hline
\end{tabular}

Source: Castleberry, RP. Neuroblastoma, Eur J Cancer, 33: 1430-1437, 1997. 
with neuroblastoma. Specifically, the 5-year event-free survival for 12- to 18-month old children was more than $70-92 \%$ compared to $31-38 \%$ for older children $(27,28,29)$.

\subsubsection{MYCN amplification as a prognostic indicator}

The oncogene MYCN located on chromosome 2p23-24 was first identified in neuroblastomas (30), but is also amplified and overexpressed in other tumors including retinoblastoma (31) and small-cell lung cancer (32). MYCN amplification is associated with advanced stage neuroblastoma and rapid disease progression. Although the precise molecular reasons for the association between $M Y C N$ amplification and poor prognosis have not been determined, some of the MYCN target genes are involved in cell growth and cell cycle progression (33). Cells overexpressing MYCN have shortened cell cycle times (34). MYCN overexpression is also associated with increased metastatic potential via reduced attachment to extracellular matrix (35).

Normally MYCN is expressed in the developing nervous system. N-Myc, the protein product of the MYCN gene, localizes to the nucleus. N-Myc recognizes E-box motifs in the promoter region of target genes and activates transcription $(33,36)$. N-Myc target genes include several $\mathrm{ABC}$ transporters which efflux many of the drugs used in neuroblastoma therapy (reviewed in section 1.3).

Norris and colleagues evaluated the relationship between several prognostic indicators in neuroblastoma and outcome, including MYCN amplification, age, tumor stage, and MRP1 expression (10). High MRP1 mRNA expression was the prognostic indicator most closely associated with outcome. In particular, high MRP1 levels predicted reduced event-free and overall survival in children with neuroblastoma. After adjusting outcome for MYCN amplification in multivariate analysis, $M R P 1$ expression remained an 
independent predictor of survival. Due to the prominence of MYCN amplification in neuroblastoma and the molecular relationship between $\mathrm{N}-\mathrm{Myc}$ and $\mathrm{ABC}$ transporter expression, the association between MYCN amplification and poor prognosis may be due to reduced intracellular accumulation of drugs.

\subsubsection{Shimada Histology as a prognostic indicator}

The histopathologic features of neuroblastoma can also be used as prognostic indicators. Neuroblastoma histopathology consists of diffuse neuroblastic cell clusters with irregular fibrovascular separations. The Shimada histology classification system was proposed upon retrospective evaluation of untreated primary neuroblastoma specimens from 295 patients for whom at least 2 years of follow-up data were available (37). This system includes evaluation of stromal development and neuroblastic cell maturation/ differentiation. Initially specimens are classified as stroma-rich or stroma-poor. Stromarich tumors are divided into 3 subgroups: well-differentiated, intermixed, and nodular. Both well-differentiated and intermixed tumors are classified as favorable $(37,38,39)$. Lastly, a nodular appearance confers unfavorable histology. Favorable or unfavorable histology in stroma-poor cases depends on neuroblast differentiation, mitosiskaryorrhexis index (MKI), and age at diagnosis.

\subsubsection{DNA Index}

DNA Index is another feature of neuroblastoma used in risk group stratification to guide therapy for children with neuroblastoma. DNA Index (also called DNA ploidy) is a measure of tumor DNA content, which is measured by FACS analysis of propidium iodide stained nuclei. Aneuploidy is considered a favorable clinical prognostic factor in 
neuroblastoma. Patients with diploid or tetraploid tumors have a poor prognosis in comparison to those with triploid tumors $(40,41,42,43)$.

\subsubsection{Risk group stratification}

Heterogeneity is a hallmark of neuroblastoma as demonstrated by variations in age at diagnosis, DNA index, tumor histopathology, MYCN amplification, and other biological features in neuroblastoma. Based on the heterogeneous survival rates for children diagnosed with neuroblastoma, the Pediatric Oncology Group (POG) and Children's Cancer Group designed a risk stratification system to guide risk-related therapy based upon prognosis (2). Stratification is based on five prognostic factors: INSS stage, age at diagnosis, MYCN status, Shimada histology, and DNA index (Table 1.1). This system stratifies children diagnosed with neuroblastoma into three risk groups: low, intermediate, and high-risk disease.

\subsubsection{Risk-adapted therapy}

\subsubsection{Low risk patients}

Patients with low risk disease have greater than 95\% predicted event-free survival $(2,44)$. Low risk neuroblastoma includes all patients with Stage 1 (localized) disease. Localized disease is present in approximately $40 \%$ of neuroblastoma cases. In addition, all children with Stage 2 disease are classified as low risk except those older than $1 \mathrm{yr}$ with MYCN amplification and unfavorable histology. In a POG study of 101 children with localized low risk disease treated with surgery as the sole treatment modality, only 9 patients relapsed (44). Therefore, children with low risk localized disease should undergo 
surgical resection of the tumor. However, if local recurrence of the tumor is observed, these patients can be treated with further surgery or chemotherapy.

\subsubsection{Intermediate risk patients}

Patients with intermediate risk disease have a 3-year predicted event-free survival greater than $85 \%$ (2). Children with intermediate risk disease are stratified according to Table 1.1. Therapy for intermediate risk disease includes surgery and chemotherapy. Despite the favorable outcome for most patients with intermediate risk neuroblastoma, advancements must be made in therapy to reduce acute and long-term toxicities.

\subsubsection{High-risk patients}

The focus of this dissertation will remain on the contribution of $\mathrm{ABC}$ transporters to camptothecin analog resistance in high-risk neuroblastoma. Children diagnosed with high-risk neuroblastoma have less than $30 \%$ predicted event-free survival. Children with high-risk disease are stratified according to Table 1.1. Currently children diagnosed with high-risk neuroblastoma receive multiple modalities of therapy including cytotoxic chemotherapy, surgery, radiation, and bone marrow transplant.

The current protocol approach to high-risk neuroblastoma therapy includes induction, continuation, and maintenance phases. The goal of induction is to reduce the tumor mass by combining agents such as cisplatin, doxorubicin, etoposide, vincristine, and cyclophosphamide (45). These five drugs are combined to take advantage of different mechanisms of action and non-overlapping toxicities. Moreover, in accordance with the Goldie-Coldman hypothesis, the risk of developing drug resistance is decreased by the use of non-cross-resistant chemotherapeutic agents to achieve rapid cytoreduction (46). In the second phase patients may receive continuation therapy that includes the same 
agents used during induction. Alternatively, a myeloablative preparative regimen (e.g., melphalan, etoposide, and carboplatin or melphalan and total body irradiation) followed by autologous stem cell transplantation $(47,48)$ can be used to eradicate residual disease. Due to the high relapse rate in children with high-risk neuroblastoma, the goal of maintenance therapy is to eliminate minimal residual disease. Currently the differentiation inducing agent 13-cis-retinoic acid (isotretinoin) is used in maintenance therapy for high-risk neuroblastoma (48).

Due to the extremely poor outcome in children diagnosed with high-risk neuroblastoma, several approaches to improving therapy have been investigated recently or are currently in development. New therapeutic modalities such as ${ }^{131}$ I-MIBG (norepinephrine analog that concentrates in sympathetic tissue) or anti-GD2 (antigen highly expressed in neuroectodermal tumors) antibodies are under investigation. For drugs with wide variability in pharmacokinetics such as topotecan, approaches to minimize interindividual variability or to achieve the putatively effective systemic exposure have been evaluated. For example, the pharmacokinetically guided approach to topotecan dosing in children with high-risk neuroblastoma showed an excellent response to single agent topotecan targeted to the putatively effective systemic exposure $(49,15)$. However, chemotherapeutic agents are rarely used as single agents. Due to the favorable outcome in some children with neuroblastoma, pharmacokinetically guided topotecan has also been evaluated in combination with other drugs active in neuroblastoma such as cyclophosphamide.

Despite the extremely favorable response to topotecan in children with high-risk neuroblastoma ( $60 \%$ response rate), the lack of a response in $40 \%$ of the children treated 
with pharmacokinetically guided topotecan indicates clinical resistance to topotecan in neuroblastoma (15). The mechanisms for this resistance are not completely understood. Identifying the molecular determinants of resistance in children with either intrinsic resistance at therapy initiation or acquired resistance after several cycles of chemotherapy may help to guide selection of the most active treatment regimens for individual patients.

Resistance in neuroblastoma is likely to be a multifactorial phenomenon. For the camptothecin analogs, mutations in drug target (i.e., topoisomerase I) can lead to resistance $(50,51)$. In addition, resistance can be caused by increased intracellular detoxification and alterations in cell death or survival pathways. Finally, ABC transporters that efflux camptothecin analogs can confer resistance.

The studies of $\mathrm{ABC}$ transporters in neuroblastoma have generated controversial and conflicting results. High levels of MRP1 mRNA expression in primary, untreated neuroblastoma are associated with reduced survival and event-free survival in patients with neuroblastoma (10). Moreover, in tumors without MYCN amplification, high levels of MDR1 mRNA expression are associated with poor cumulative survival and event-free survival (11). However, the prognostic significance of P-glycoprotein expression, which is encoded by MDR1, in neuroblastoma remains controversial (52). For example, de Cremoux and colleagues have demonstrated that high levels of MRP1 or MDR1 did not predict neuroblastoma response in a study of biopsy samples from 29 high-risk patients (53). In contrast Norris and colleagues have demonstrated that MRP1 is a significant, independent predictor of poor prognosis in a prospective analysis of a large group of children with neuroblastoma (10). Despite the controversial results of studies of the relation between $\mathrm{ABC}$ transporter expression in neuroblastoma and clinical outcome, the 
drugs that are subject to $\mathrm{ABC}$ transporter-mediated resistance are used in the treatment of neuroblastoma. For example, the camptothecin analogs topotecan and irinotecan have anti-tumor activity in children with neuroblastoma, but these drugs are substrates for several of the $A B C$ transporters. Elucidation of the role of $A B C$ transporters in resistance of primary tumors to topotecan and irinotecan may improve our ability to obtain the maximum therapeutic benefit of topotecan and irinotecan in treating neuroblastoma

\subsection{Camptothecin analogs are topoisomerase I interactive agents}

Camptothecin is a naturally occurring alkaloid isolated from the Camptotheca acuminata tree (54). Despite demonstrated anti-tumor activity, the clinical utility of camptothecin sodium is limited due to severe toxicity, including hemorrhagic cystitis characterized by necrotic ulcerations throughout the urinary tract $(55,56,57)$. Other toxicities associated with camptothecin sodium include nausea, vomiting, alopecia, stomatitis, and diarrhea. The dose limiting toxicity (DLT) of camptothecin sodium is myelosuppression, including neutropenia and thrombocytopenia. However, due to the promising anti-tumor properties of camptothecin sodium and the $\mathrm{pH}$ dependent toxicity in the urinary bladder, more water-soluble camptothecin analogs topotecan and irinotecan were synthesized. Results of early clinical trials with these compounds demonstrated a more acceptable toxicity profile and retention of the promising anti-tumor activity of the parent compound. Figure 1.1 shows chemical structures of the parent pentacyclic camptothecin as well as topotecan, irinotecan, and $\mathrm{SN}-38$ (the active metabolite of the prodrug irinotecan). The clinical utility of both topotecan and irinotecan has been demonstrated in pre-clinical and clinical studies of several pediatric tumor models, including neuroblastoma. 

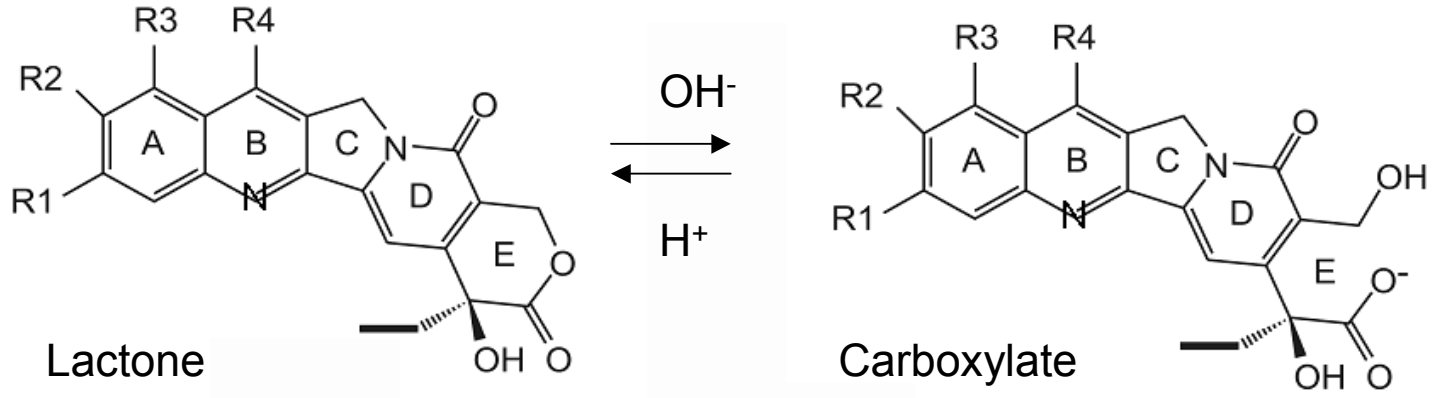

Figure 1.1 Camptothecin analog chemical structure.

\begin{tabular}{|c|c|c|c|c|}
\hline & R1 & R2 & R3 & $\mathrm{R} 4$ \\
\hline Camptothecin & $\mathrm{H}$ & $\mathrm{H}$ & $\mathrm{H}$ & $\mathrm{H}$ \\
\hline $\begin{array}{l}\text { Topotecan } \\
\text { (TPT) }\end{array}$ & $\mathrm{H}$ & $\mathrm{OH}$ & $-\mathrm{CH}_{2} \mathrm{~N}\left(\mathrm{CH}_{3}\right)_{2}$ & $\mathrm{H}$ \\
\hline $\begin{array}{l}\text { Irinotecan } \\
\text { (IRN, CPT-11) }\end{array}$ & $\mathrm{H}$ & & $\mathrm{H}$ & $-\mathrm{CH}_{2} \mathrm{CH}_{3}$ \\
\hline SN-38 & $\mathrm{H}$ & $\mathrm{OH}$ & $\mathrm{H}$ & $-\mathrm{CH}_{2} \mathrm{CH}_{3}$ \\
\hline
\end{tabular}

Camptothecin analogs undergo reversible $\mathrm{pH}$-dependent conversion between the openring carboxylate form and closed-ring lactone form. At physiologic $\mathrm{pH}$ (7.4) the carboxylate form of the camptothecin analog predominates; whereas, the lactone form predominates under acidic conditions. The camptothecin analog lactone form is responsible for the pharmacological activity at the site of action by interacting with the target enzyme topoisomerase I. 
Topoisomerase I is the molecular target of camptothecin analogs. Topoisomerase I relieves torsional strain in DNA by cleaving of a single DNA strand, permitting the second DNA strand to pass through the break, and then religating the cleaved strand. Camptothecin analogs stabilize these transient DNA-topoisomerase I complexes during DNA synthesis. The replication fork arrests upon collision with the resultant covalent DNA-topoisomerase I complexes, which are thought to generate lethal DNA strand breaks (58).

At physiological $\mathrm{pH}$ the $\alpha$-hydroxylactone E-ring ring system undergoes reversible hydrolysis to form the anionic carboxylic acid moiety (58). In vitro studies of camptothecin analogs have demonstrated that the pharmacologically active form is the closed ring lactone form (59). The open E-ring carboxylate conformation has little if any cytotoxic effect (60). Because the lactone form of camptothecin analogs is considered the active conformer, phase I and II trials in children have extensively investigated the pharmacokinetics of topotecan lactone and irinotecan/SN-38 lactone.

\subsubsection{Topotecan}

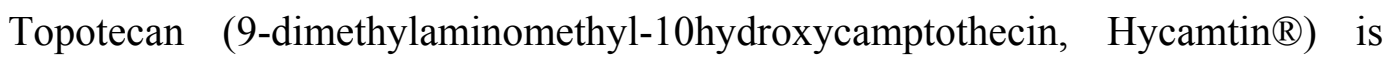
FDA-approved for treatment of ovarian cancer and small cell lung cancer in adults. Preclinical xenograft studies in mice have demonstrated activity of protracted topotecan administration for treatment of several pediatric solid tumors including neuroblastoma, medulloblastoma, glioblastoma, and rhabdomyosarcoma $(61,62)$. Importantly, a steep topotecan lactone systemic exposure- response relationship has been documented. Zamboni and colleagues investigated the relationship between topotecan systemic exposure and response in a panel of six neuroblastoma xenograft models (61). Mice 
bearing subcutaneous neuroblastoma xenografts were treated with a range of topotecan dosages ( 0.36 to $2 \mathrm{mg} / \mathrm{kg})$ daily for 5 days for 2 weeks [(dx5)2] every 21 days for 3 courses. The investigators determined the topotecan lactone systemic exposure associated with response in a panel of six neuroblastoma xenografts (i.e., NB1382.2, NB1643, NB1691, NB1771, NBEB, and NBSD). Four of the 6 neuroblastoma xenograft models demonstrated a complete response to topotecan dosages associated with a topotecan lactone area under the concentration-time curve (AUC) less than or equal to 88 $\mathrm{ng} / \mathrm{mL}^{*} \mathrm{hr}$. Both in vivo and in vitro NB1643 was relatively sensitive to topotecan; whereas, NB1691 required a topotecan lactone systemic exposure of $290 \mathrm{ng} / \mathrm{mL} * \mathrm{hr}$ to achieve a complete response (63). Despite the resistance of NB1691 to topotecan both in vitro and in vivo, the favorable response of four other neuroblastoma xenograft models to topotecan provided a strong basis for further study of topotecan in neuroblastoma clinical trials.

Several topotecan dosage schedules have been evaluated in phase I and II clinical trials in children. A $72 \mathrm{hr}$ continuous topotecan infusion showed minimal anti-tumor activity in neuroblastoma (Table 1.3). A five-day schedule of topotecan was minimally active in neuroblastoma, but interindividual variability in topotecan pharmacokinetics was high $(66,67)$.

However, topotecan is an S-phase selective agent. Maximizing drug exposure to cells (particularly those with long cell-cycle times and low growth fractions) in S-phase can be achieved using protracted administration schedules such as daily for five days for two to three weeks (68). Studies of topotecan in xenograft models demonstrated the superiority of protracted schedules of administration over high doses administered 
Table 1.3 Topotecan clinical trials in children with recurrent or refractory solid tumors including neuroblastoma.

\begin{tabular}{|c|c|c|c|c|c|c|c|}
\hline Phase & Dosage & $\begin{array}{c}\mathrm{N} \\
\text { (n with } \\
\text { NB) }\end{array}$ & $\begin{array}{c}\text { MTD } \\
\left(\mathrm{mg} / \mathrm{m}^{2} / \mathrm{d}\right)\end{array}$ & DLT & $\begin{array}{c}\text { Responses } \\
\text { in children } \\
\text { with NB }\end{array}$ & Notes & Ref. \\
\hline $\bar{I}$ & $72 \mathrm{hr} \mathrm{CI}$ & $27(9)$ & $\begin{array}{c}1.0 \\
\text { with G- } \\
\text { CSF }\end{array}$ & Myelosuppression & $\begin{array}{l}1 \mathrm{CR} \text { at } 1.3 \\
\mathrm{mg} / \mathrm{m}^{2} / \text { day } \\
\text { maintained } \\
\text { for } 8 \\
\text { months; } 5 \\
\mathrm{SD}\end{array}$ & & $(64)$ \\
\hline II & $72 \mathrm{hr} \mathrm{CI}$ & $\begin{array}{l}93 \text { (not } \\
\text { reported) }\end{array}$ & 1.3 & Myelosuppression & $1 \mathrm{CR}$ & $\begin{array}{l}\text { Schedule } \\
\text { inactive } \\
\text { in } \\
\text { recurrent/ } \\
\text { refractory } \\
\text { NB }\end{array}$ & $(65)$ \\
\hline I & $\begin{array}{c}\text { Dx5 } \\
\text { Q 21d } \\
30 \text { min } \\
\text { i.v. } \\
\text { infusion }\end{array}$ & $40(9)$ & $\begin{array}{c}1.4 \\
\text { without } \\
\text { G-CSF; } \\
2.0 \\
\text { with G- } \\
\text { CSF }\end{array}$ & $\begin{array}{l}\text { Neutropenia, } \\
\text { thrombocytopenia }\end{array}$ & 3 PR & $\begin{array}{l}7 \text {-fold } \\
\text { range in } \\
\text { topotecan } \\
\text { lactone } \\
\text { systemic } \\
\text { clearance }\end{array}$ & (66) \\
\hline II & $\begin{array}{c}\text { Dx5 Q } \\
21 \mathrm{~d} \text { by } \\
30 \mathrm{~min} \\
\text { i.v. } \\
\text { infusion }\end{array}$ & $20(13)$ & 1.48 & $\begin{array}{c}\text { Anemia, } \\
\text { neutropenia, } \\
\text { thrombocytopenia }\end{array}$ & $2 \mathrm{PR} ; 4 \mathrm{SD}$ & & (67) \\
\hline
\end{tabular}

Abbreviations: NB- neuroblastoma, MTD- maximum tolerated dose, DLT- dose limiting toxicity, CI- continuous infusion, G-CSF- granulocyte colony stimulating factor, CRcomplete response, Dx5 Q 21 d- daily for 5 days every 21 days, PR- partial response, SDstable disease

Sources: Pratt CB, et al. Phase I study of topotecan for pediatric patients with malignant solid tumors, J Clin Oncol, 12: 539-543, 1994.

Blaney SM, et al. Phase II trial of topotecan administered as 72-hour continuous infusion in children with refractory solid tumors: a collaborative Pediatric Branch, National Cancer Institute, and Children's Cancer Group Study, Clin Cancer Res, 4: 357-360, 1998.

Tubergen DG, et al. Phase I trial and pharmacokinetic (PK) and pharmacodynamics (PD) study of topotecan using a five-day course in children with refractory solid tumors: A Pediatric Oncology Group Study, J Ped Hem/Onc, 18: 352-361, 1996.

Langler A, et al. Topotecan in the treatment of refractory neuroblastoma and other malignant tumors in childhood - a phase-II-study, Klin Padiatr, 214: 153-156, 2002. 
Intermittently (68). Based on the strength of these preclinical data, Santana and colleagues evaluated the protracted administration with pharmacokinetically guided dosing of topotecan to minimize variability in systemic exposure and achieve the putatively effective topotecan systemic exposure in children.

Santana and colleagues conducted a pilot study of protracted topotecan in children with refractory solid tumors (Table 1.4) (49). Due to the wide interpatient variability in topotecan pharmacokinetics and the documented relationship between topotecan systemic exposure and response, the investigators used a pharmacokinetically guided dosing approach to minimize interpatient variation in topotecan systemic exposure. This approach also removes interindividual variability as a factor contributing to resistance. The single day topotecan lactone systemic exposure evaluated in children was based upon anti-tumor response in xenograft models at topotecan lactone AUC ranging from 88 to $144 \mathrm{ng} / \mathrm{mL}^{*} \mathrm{hr}(68,66,61)$.

In the pilot Phase I trial the pharmacokinetically guided dosing resulted in achieving the targeted systemic exposure in $78 \%$ (46 of 59) of the pharmacokinetic studies (49). The investigators also demonstrated a significant reduction in AUC values outside of the targeted range in comparison to that which would be achieved using a fixed dosing approach based solely on body surface area. Due to the manageable toxicity and favorable responses to pharmacokinetically guided dosing of protracted topotecan, a prospective phase II trial was conducted in previously untreated high-risk neuroblastoma (Table 1.4) (15). The pharmacokinetic targeting success rate was $72 \%$, indicating the superiority of this individualized approach to topotecan dosing over fixed dosing to achieve a desired AUC. 
Table 1.4 Clinical trials of topotecan pharmacokinetically guided dosing in children with solid tumors including neuroblastoma.

\begin{tabular}{|c|c|c|c|c|c|c|}
\hline Phase & $\begin{array}{c}\text { Dosage } \\
\text { Schedule }\end{array}$ & $\begin{array}{l}\mathrm{N}(\mathrm{n} \\
\text { with } \\
\mathrm{NB})\end{array}$ & Population & $\begin{array}{c}\text { Single Day } \\
\text { Topotecan } \\
\text { Lactone } \\
\text { Target } \\
\text { (ng/mL*hr) }\end{array}$ & $\begin{array}{c}\text { NB } \\
\text { Responses }\end{array}$ & Reference \\
\hline $\bar{I}$ & $\begin{array}{l}\text { (Dx5)2 } \\
\text { Q 24-28 d } \\
30 \text { min } \\
\text { i.v. infusion }\end{array}$ & $15(5)$ & $\begin{array}{l}\text { Recurrent/ } \\
\text { refractory } \\
\text { solid tumors }\end{array}$ & $\begin{array}{l}\text { Cohort 1: } \\
120-160 \\
\text { Cohort 2: } \\
80-120\end{array}$ & $\begin{array}{l}\text { Cohort 1: } \\
1 \text { PR } \\
\text { Cohort 2: } \\
1 \text { PR }\end{array}$ & (49) \\
\hline II & $\begin{array}{l}(\mathrm{Dx} 5) 2 \\
\text { Q } 28 \mathrm{~d} \\
30 \mathrm{~min} \\
\text { i.v. infusion }\end{array}$ & $30(30)$ & $\begin{array}{l}\text { Previously } \\
\text { untreated } \\
\text { high-risk NB }\end{array}$ & $80-120$ & $\begin{array}{l}1 \mathrm{CR} \\
17 \mathrm{PR}\end{array}$ & (15) \\
\hline
\end{tabular}

Abbreviations: NB- neuroblastoma, (Dx5)2- daily for 5 days for 2 weeks, Q 24-28 devery 24 to 28 days, i.v.- intravenous, Q 28 d, every 28 days, PR- partial response, CRcomplete response

Sources: Santana VM, et al. A pilot study of protracted topotecan dosing using a pharmacokinetically guided dosing approach in children with solid tumors, Clin Cancer Res, 9: 633-640, 2003.

Santana VM, et al. Improved response in high-risk neuroblastoma with protracted topotecan administration using a pharmacokinetically guided dosing approach, J Clin Oncol, 23: 4039-4047, 2005. 
The feasibility of pharmacokinetically guided topotecan dosing determined in the pilot study was confirmed in this phase II study. The $60 \%$ response rate (complete and partial responses) was excellent in these previously untreated patients, especially in comparison to studies in relapse patients where the response rate was only $15 \%$ (67). The $60 \%$ response rate to pharmacokinetically guided topotecan was also more favorable than the $40 \%$ response rate observed in a Phase II POG study of topotecan $(2 \mathrm{mg} / \mathrm{kg}$ administered dx5) in 33 children with previously untreated Stage IV neuroblastoma (3). The higher response rate in the Santana study might be due to several factors including number of days of topotecan, dosage of topotecan, and systemic exposure of topotecan. The patients with refractory tumors on the Langler study (67) might represent a more drug resistant disease subset than the previously untreated population in the Santana study. In the Langler and POG (3) studies, patients received only 5 days of topotecan rather than 10 days over 2 weeks. Moreover, the daily topotecan dosages were lower in the Langler and (median 1.48, range $0.4-1.7 \mathrm{mg} / \mathrm{m} 2)$ and POG studies $(2 \mathrm{mg} / \mathrm{m} 2)$ than in the Santana study (median 2.7, range 0.95-3.8 $\mathrm{mg} / \mathrm{m} 2$ ). However, since topotecan pharmacokinetics were not reported in the Langler or POG studies, direct comparison of topotecan pharmacokinetics cannot be made to those reported by Santana and colleagues. Based on the documented interindividual variability in topotecan pharmacokinetics, substantial variation in topotecan systemic exposure was likely present in the studies using a fixed dosing approach. The approach to topotecan fixed dosing for 5 days every 21 days without dosage adjustment based on a targeted systemic exposure is probably subtherapeutic, especially in comparison to the protracted topotecan schedule targeting a systemic exposure of $80-120 \mathrm{ng} / \mathrm{mL}^{*} \mathrm{hr}$. Based on the systemic exposure-response 
relationship, interindividual variability in drug exposure likely contributes to some cases of apparent topotecan resistance. However, the pharmacokinetic guided topotecan dosing approach minimizes interindividual variability in topotecan systemic exposure.

Although protracted topotecan with pharmacokinetically guided dosing adjustments to achieve an AUC of $80-120 \mathrm{ng} / \mathrm{mL} * \mathrm{hr}$ resulted in an excellent response rate in chemotherapy naïve children diagnosed with neuroblastoma, the tumor response rate $(60 \%)$ was lower than the pharmacokinetic targeting success $(72 \%)$. The patients who did not respond to pharmacokinetically guided protracted topotecan represent a subset of the disease that is clinically resistant to topotecan.

Resistance to topotecan can be multifactorial. Reduced expression of topoisomerase I, the molecular target of topotecan, can result in topotecan resistance. Topoisomerase I mutations that affect topotecan binding may confer topotecan resistance (50,51). Increased DNA repair via tyrosyl-DNA-phosphodiesterase overexpression can also lead to camptothecin analog resistance(69). Finally, ABC transporter-mediated efflux from the cell may result in topotecan resistance.

\subsubsection{Irinotecan}

Irinotecan (7-ethyl-10-(4-[1-piperidino]-1-piperidino)-carbonyloxy-camptothecin, Camptosar ${ }^{\circledR}$ ), a camptothecin analog, has been evaluated in a variety of pediatric tumors, including neuroblastoma. The prodrug irinotecan has been FDA approved for treatment of metastatic colorectal cancer. Irinotecan is metabolized by carboxylesterases to the active metabolite SN-38. Preclinical and clinical studies have demonstrated that i.v. irinotecan was also active against pediatric tumors including rhabdomyosarcoma, 
medulloblastoma, glioblastoma, and neuroblastoma $(62,70)$. Oral irinotecan was active in a panel of six neuroblastoma xenograft models (71).

Vassal and colleagues demonstrated irinotecan activity in a panel of three neuroblastoma xenografts established from patients with poor prognosis (72). IGR-N835 was established from a previously treated patient with MYCN amplified stage IV disease lacking MDR1 expression. IGR-NB3 and IGR-NB8 were derived from two patients with untreated stage III disease, MYCN amplification, and MDR1 overexpression, each of which was refractory to chemotherapy. A complete or partial response to irinotecan (either using a dx5 or $\mathrm{q} 4 \mathrm{dx} 5$ administration schedule) was achieved in all 3 xenograft models studied. Although several investigators have demonstrated resistance to irinotecan and SN-38 in Pgp overexpressing cell lines in vitro $(73,74)$, the response to irinotecan of xenografts overexpressing Pgp suggests that Pgp did not play a major role in irinotecan resistance in this model. However, to confirm this hypothesis it would be necessary to demonstrate irinotecan efficacy in patients with neuroblastoma that overexpresses Pgp.

In contrast to the $\mathrm{dx} 5$ irinotecan dosing approach reported by Vassal et al, Thompson and colleagues demonstrated the anti-tumor activity of protracted (i.e., daily for 5 days for 2 weeks) i.v. irinotecan in a panel of 6 neuroblastoma xenografts (70). The characteristics of each xenograft model are listed in Table 1.5. A single course of irinotecan $10 \mathrm{mg} / \mathrm{kg}$ daily for 5 days for 2 weeks induced a complete response in NB1691, NB1643, NBEB, and NB1382.2 tumors. Also, a partial response was achieved in NB1771. However, NBSD, which was derived from a bone marrow aspirate, did not respond significantly to this irinotecan dosage. This might indicate that neuroblastoma bone marrow metastases are more refractory to irinotecan than the primary tumor. The 
Table 1.5 Neuroblastoma xenograft features.

\begin{tabular}{|c|c|c|c|c|}
\hline Xenograft & Stage & Site & Prior Therapy & $\begin{array}{c}\text { MYCN } \\
\text { Amplification }\end{array}$ \\
\hline NB1691 & $\mathrm{D}$ & Adrenal & $\begin{array}{l}\text { AraC, Dauno, 6-TG, VP-16, } \\
\text { 5-azacytidine }\end{array}$ & Yes \\
\hline NB1643 & $\mathrm{D}$ & Retro-peritoneal & None & Yes \\
\hline NB1382.2 & $\mathrm{C}$ & Retro-peritoneal & $\begin{array}{l}\text { VCR, VP-16, CTX, CDDP, } \\
\text { carboplatin }\end{array}$ & Yes \\
\hline NB1771 & $\mathrm{D}$ & Adrenal & None & Yes \\
\hline NBSD & $\mathrm{D}$ & $\begin{array}{l}\text { Bone marrow } \\
\text { aspirate }\end{array}$ & CTX, Dox, CDDP, VM-26 & Yes \\
\hline NBEB & $\mathrm{D}$ & Adrenal & CTX, Dox,CDDP,VM-26 & No \\
\hline
\end{tabular}

Abbreviations: AraC- cytosine arabinoside, Dauno- daunorubicin, 6-TG- 6-thioguanine, VP-16- etoposide, VCR- vincristine, CTX- cyclophosphamide, CDDP- cisplatin, Doxdoxorubicin, VM-26- teniposide. (Note: stage D is equivalent to current INSS stage 4.)

Source: Thompson J, et al. Efficacy of systemic administration of irinotecan against neuroblastoma xenografts, Clin Cancer Res, 3: 423-431, 1997. 
investigators also determined that the irinotecan and $\mathrm{SN}-38$ systemic exposures associated with the minimum effective dose in xenograft models can also be achieved clinically in children with cancer (17). Furthermore, at systemic exposures tolerated in children (75) oral irinotecan elicited anti-tumor responses in the same panel of neuroblastoma xenografts (71).

Several schedules of irinotecan administration have been evaluated in children with solid tumors in phase I trials (Table 1.6). The major toxicities associated with irinotecan were myelosuppression and diarrhea. Favorable responses were observed in several tumor types, for neuroblastoma in particular. Based solely on the phase I clinical data, the optimal irinotecan schedule cannot be determined unequivocally. However, the extensive preclinical studies of Houghton and colleagues have demonstrated the superiority of protracted irinotecan to shorter schedules. Moreover, due to the potential for failure to respond to irinotecan, future clinical trials of irinotecan should include prospective evaluation of factors that might influence anti-tumor response, including expression of $\mathrm{ABC}$ transporters in the tumor.

\subsection{ABC transporters}

The human ABC superfamily of transmembrane proteins contains 49 genes divided into 7 subfamilies (ABCA through ABCG) based upon gene structure, order of domains, and amino acid sequence homology (79). ABC transporters are expressed in both normal and tumor tissues and efflux a variety of endogenous substrates and xenobiotics from cells against a concentration gradient. $\mathrm{ABC}$ transporters expressed in normal tissues protect cells from toxic substances. ABC transporters expressed in normal tissues also play a role in the pharmacokinetics (absorption, distribution, metabolism, and 
Table 1.6 Irinotecan phase I clinical trials in children with recurrent or refractory solid tumors including neuroblastoma.

\begin{tabular}{|c|c|c|c|c|}
\hline $\begin{array}{l}\text { Dosage } \\
\text { Schedule }\end{array}$ & $\begin{array}{c}\mathrm{N} \\
\text { (n with NB) }\end{array}$ & MTD, DLT & $\begin{array}{c}\text { Responses in } \\
\text { children with } \\
\text { NB } \\
\end{array}$ & Reference \\
\hline $\begin{array}{l}(\mathrm{Dx} 5) 2 \text { by } 1 \\
\text { hr i.v. } \\
\text { infusion }\end{array}$ & $23(5)$ & $20 \mathrm{mg} / \mathrm{m}^{2} /$ day, diarrhea & $1 \mathrm{PR}$ & $(17)$ \\
\hline $\begin{array}{l}\text { Dx } 5 \text { q } 21 \mathrm{~d} \\
\text { by } 1 \mathrm{hr} \text { i.v. } \\
\text { infusion }\end{array}$ & $35(7)$ & $\begin{array}{l}\text { Heavily pretreated } \\
\text { stratum: } 39 \mathrm{mg} / \mathrm{m}^{2} / \text { day, } \\
\text { neutropenia/ } \\
\text { thrombocytopenia; } \\
\text { Less heavily pretreated } \\
\text { stratum: } 50 \mathrm{mg} / \mathrm{m}^{2} / \text { day, } \\
\text { diarrhea }\end{array}$ & $1 \mathrm{PR}, 1 \mathrm{SD}$ & $(76)$ \\
\hline $\begin{array}{l}\text { QW for } 3 \mathrm{~W} \\
\text { by } 1 \text { or } 2 \mathrm{hr} \\
\text { i.v. infusion }\end{array}$ & $\begin{array}{l}\text { Less heavily } \\
\text { pretreated: } \\
48(5) \\
\text { More heavily } \\
\text { pretreated: } \\
33(11)\end{array}$ & $\begin{array}{l}600 \mathrm{mg} / \mathrm{m}^{2} / \text { dose, delayed } \\
\text { diarrhea and cholinergic } \\
\text { syndrome for less } \\
\text { heavily pretreated; } \\
\text { neutropenia for heavily } \\
\text { pretreated }\end{array}$ & $\begin{array}{l}1 \mathrm{PR} \\
\text { persisted for } \\
5 \text { courses }\end{array}$ & $(77)$ \\
\hline $\begin{array}{l}\text { Dx } 3 \text { by } 2 \mathrm{hr} \\
\text { i.v. infusion } \\
\text { q } 28 \mathrm{~d}\end{array}$ & $28(26)$ & $\begin{array}{l}160-180 \mathrm{mg} / \mathrm{m}^{2} / \text { day, } \\
\text { myelosuppression } \\
\text { (hematopoietic lineages) } \\
\text { and diarrhea }\end{array}$ & $4 \mathrm{PR}$ & $(78)$ \\
\hline
\end{tabular}

Abbreviations: NB- neuroblastoma, MTD- maximum tolerated dose, DLT- dose limiting toxicity, (Dx5)2- daily for 5 days for 2 weeks, Dx 5 q 21 d- daily for 5 days every 21 days, QW- every week, 3W- 3 weeks, PR-partial response, SD- stable disease

Sources: Furman WL, et al. Direct translation of a protracted irinotecan schedule from a xenograft model to a phase I trial in children, J Clin Oncol, 17: 1815-1824, 1999.

Blaney S, et al. A phase I study of irinotecan in pediatric patients: a pediatric oncology group study, Clin Cancer Res, 7: 32-37, 2001.

Vassal G, et al. A phase I study of irinotecan as a 3-week schedule in children with refractory or recurrent solid tumors, J Clin Oncol, 21: 3844-3852, 2003.

Mugishima $\mathrm{H}$, et al. Phase I study of irinotecan in pediatric patients with malignant solid tumors, J Pediatr Hematol Oncol, 24: 94-100, 2002. 
excretion) of drugs that are $\mathrm{ABC}$ transporter substrates. Finally, multi-drug resistance via drug efflux is one of the functional consequences of $\mathrm{ABC}$ transporter expression in malignant cells.

ATP binding or hydrolysis drives this efflux process. Several mechanisms for extruding substrates from a cell have been proposed, including those of a classical pump, a flippase, a hydrophobic vacuum cleaner, and an ATP switch. According to the classical pump model, $\mathrm{ABC}$ transporters remove substrates directly from the cytoplasm to the extracellular space. However, because substrates have been shown to enter the transporter via the membrane and not the cytoplasm, $\mathrm{ABC}$ transporters likely function according to an alternate mechanism (80). According to the flippase model, a transporter flips a substrate from the inner membrane leaflet to the outer membrane leaflet from which the substrate could diffuse into the extracellular environment (81). The hydrophobic vacuum cleaner model proposes that the transmembrane domains associate to form a channel in the plasma membrane (82). Upon passive diffusion of a substrate into the lipid bilayer, the transporters detect substrates in the membrane and efflux them from the cell. The ATP switch model is similar to the hydrophobic vacuum cleaner model. Upon substrate binding to a high-affinity site on the transmembrane domain (TMD) of the inner membrane leaflet, two ATP molecules bind the nucleotide binding domain (NBD), inducing a conformational change in the TMD (83). This conformational change exposes the substrate to the extracellular space, and the affinity of the transporter for the substrate is reduced. Hence, the bound drug is released into the extracellular space. Following ATP hydrolysis, inorganic phosphate and ADP are released, and the transporter is restored to its basal conformation. 
In addition to similar mechanisms of action, $\mathrm{ABC}$ transporters share structural features. Structural domains common to this protein superfamily include a TMD and a highly conserved ATP-binding cassette, which is also called the nucleotide binding domain (NBD). The ATP-binding domains known as Walker A and Walker B motifs in the NBD are separated by $90-120$ amino acids (79). A functional transporter is comprised of at least 2 TMDs, each of which contains 6-11 membrane spanning $\alpha$-helices.

The ABC transporters Pgp, MRP1, MRP2, MRP4, and BCRP may play a role in resistance to topotecan, irinotecan, and SN-38. The role of these transporters in resistance to topotecan, irinotecan, and $\mathrm{SN}-38$ in addition to what is known about their expression in neuroblastoma will be summarized below.

\subsubsection{P-glycoprotein}

P-glycoprotein (Pgp) was the first member of the $\mathrm{ABC}$ transporter superfamily described(84), and it is encoded in humans by the gene ABCB1, also known as MDR1. The protein product of the MDR1 gene is a $170 \mathrm{kDa}$ plasma membrane glycoprotein. Pgp has also been shown to be expressed in the membranes of organelles such as the nuclear envelope and Golgi apparatus $(85,86)$. Although the full-length $M D R 1$ gene was not cloned until 1986 (87), Pgp expression in multi-drug resistant cell lines was first demonstrated in 1976 (84).

Immunohistochemical analysis has demonstrated Pgp expression in various human tissues. Pgp was expressed in the epithelium of the gastrointestinal tract and bronchus, and in vascular endothelium, notably in the blood-brain barrier. Pgp was also expressed in secretory and excretory tissues including bile canaliculi and ductules, renal 
tubules, pancreatic ducts, mammary glands, prostate glands, salivary glands, and adrenal glands (88).

\subsubsection{Pgp substrates}

Pgp substrates include a wide variety of structurally diverse, hydrophobic xenobiotics including anthracyclines, anthracenes, vinca alkaloids, epipodophyllotoxins, tubulin polymerizing drugs, HIV-1 protease inhibitors, and fluorophores. Pgp substrates used in neuroblastoma therapy include doxorubicin, vincristine, etoposide, and teniposide (18). In addition, the camptothecin analogs topotecan and irinotecan, which are components of neuroblastoma therapy, have been evaluated as Pgp substrates with conflicting results $(73,89,90)$.

The first studies demonstrating that Pgp confers resistance to topotecan were conducted in drug selected cell lines. Chinese hamster ovary cells selected for colchicine resistance $\left(\mathrm{CH}^{\mathrm{R}} \mathrm{C} 5\right)$ that overexpressed Pgp were 15 times more resistant to topotecan than the parental cell line (AuxB1) and 10 times more resistant to $\mathrm{SN}-38(89,73)$. Topotecan and SN-38 accumulation and drug-stabilized topoisomerase I- DNA complexes were reduced in $\mathrm{CH}^{\mathrm{R}} \mathrm{C} 5$ compared to AuxB1, suggesting reduced intracellular topotecan or SN-38 in $\mathrm{CH}^{\mathrm{R}} \mathrm{C} 5$ due to Pgp-mediated efflux. However, these were drug selected cell lines, and expression of other transporters that might confer topotecan resistance (e.g., BCRP or MRP1) cannot be ruled out. These studies were performed prior to identification of other drug transporters; hence, the contribution of other $\mathrm{ABC}$ transporter families to the results of these studies is unknown.

In contrast to the drug selected cell lines used by Hendricks (89) and Mattern et al (73), Jansen and colleagues demonstrated irinotecan and $\mathrm{SN}-38$ resistance in cells 
transfected with MDR1 cDNA (74). A melanoma cell line transfected with MDR1 was 5 times more resistant than the parental cell line to irinotecan and only 1.4 times as resistant to $\mathrm{SN}-38$. When these same cell lines were implanted as xenografts in mice, virtually no difference was observed in sensitivity to irinotecan, indicating that the in vitro and in vivo model systems were not equivalent. Specifically, the xenografts had higher topoisomerase I activity than the cell lines in vitro, which may account for the lack of resistance observed in vivo. Similar to the results of Mattern and colleagues, the in vitro level of resistance to topotecan and SN-38 was one to two orders of magnitude lower than for the classical Pgp substrates doxorubicin and vincristine.

Pommier and colleagues used two in vitro systems to demonstrate that Pgp confers resistance to topotecan (90). First, KB-V1, a human carcinoma cell line selected for vinblastine resistance, was more resistant to camptothecin, topotecan, and SN-38 than the parental KB-3-1 cell line. In addition to the vinblastine selected cell line, camptothecin analog cytotoxicity was studied in an isogenic system, NIH3T3 and NIHMDR-G185. The resistant cell line was transfected with a plasmid encoding Pgp and maintained in colchicine. NIH-MDR-G185 showed increased resistance to topotecan, but not camptothecin or SN-38. The investigators did, however, make the important distinction that even though Pgp confers resistance to topotecan, Pgp confers a much higher level of resistance to drugs such as doxorubicin.

\subsubsection{Pgp expression in neuroblastoma}

Multidrug resistance conferred by $\mathrm{ABC}$ transporters can be either intrinsic or acquired. Intrinsic resistance of an untreated tumor can be due to expression of $\mathrm{ABC}$ transporters. Acquired resistance refers to chemotherapeutic selection of drug resistant 
clones (e.g., those expressing $\mathrm{ABC}$ transporters). Intrinsic and acquired resistance has been studied in neuroblastoma, and both may have implications for clinical outcomes including response and survival.

Pgp is expressed in many tumor types, especially those that arise from tissues that normally express Pgp. Using three monoclonal antibodies to different Pgp epitopes, Pgp expression has been demonstrated prior to chemotherapy in non-Hodgkin's lymphoma, intestinal leiomyosarcoma, adenocarcinoma of the stomach, colon, kidney, breast, and adrenal cortex (88). In addition, Fojo and colleagues demonstrated that MDR1 mRNA is expressed in the adrenal gland, adrenal medulla, and in neuroblastoma tumor samples of unspecified stage taken from 3 patients following relapse after treatment with vincristine and doxorubicin (91). Although the investigators did not evaluate the relationship between MDR1 expression and outcome, several other groups have conducted such analyses in neuroblastoma.

Results of studies evaluating the significance of Pgp mRNA or protein expression in neuroblastoma are inconsistent $(92,93,52,10)$. The inconsistencies may be explained by small study populations, differences in prior therapy, absence of paired samples at diagnosis and relapse, failure to isolate tumor tissue from normal tissue, and the potential lack of correlation between mRNA and protein expression level (94). The most reliable studies using immunohistochemistry to detect protein expression in neuroblastoma biopsies have demonstrated that overall survival is significantly lower in children with tumors that express Pgp at diagnosis (93) and Pgp expression at diagnosis increases the risk of death in neuroblastoma by $60 \%$ (52). Therefore Pgp expression in neuroblastoma 
is likely an unfavorable feature and may confer poor prognosis in neuroblastoma at least perhaps in an as yet undefined subset of patients $(93,52)$.

\subsubsection{MRP1}

The human MRP1 (ABCC1) gene contains 31 exons, maps to chromosome 16p13, and encodes a $190 \mathrm{kDa}$ protein. MRP1 was first identified in a lung cancer cell line selected for doxorubicin resistance without expression of Pgp (95). MRP1 mRNA expression was ubiquitous in a panel of human tissues $(96,97)$. In addition, western blot and immunohistochemical analysis of normal human tissues demonstrated essentially ubiquitous MRP1 expression (98). MRP1 was expressed in the adrenal gland, lung, heart, skeletal muscle, liver, spleen, kidney, stomach, small and large intestine, prostate gland, testis, ovary, and erythrocytes. However, MRP1 was not observed in endothelium.

\subsubsection{MRP1 substrates}

Some overlap exists between Pgp and MRP1 substrates. As for Pgp, MRP1 transports both endogenous and xenobiotic substrates. MRP1 substrates include glutathione S-conjugates, sulfate conjugates, and glucuronides (Table 1.7) (99). In an isogenic system Chen and colleagues demonstrated an ATP-dependent reduction in intracellular accumulation of both irinotecan and SN-38 in KB-3-1 cells transfected with MRP1 cDNA (100). Furthermore, compared to the parental cell line the MRP1 overexpressing cell line was 7 and 15 times more resistant to irinotecan and SN-38, respectively.

Allen and colleagues knocked out the murine orthologs of human MRP1 and MDR1 (Mrp1, Mdr1a, and Mdr1b respectively) in murine embryonic fibroblasts (MEF) 
Table 1.7 Partial list of endogenous and xenobiotic MRP1 substrates.

\begin{tabular}{|c|c|}
\hline Class & Substrate \\
\hline \multirow{5}{*}{ Drugs } & Methotrexate \\
\hline & Doxorubicin, daunorubicin, epirubicin, idarubicin \\
\hline & Etoposide \\
\hline & Vincristine, vinblastine \\
\hline & Irinotecan, $\mathrm{SN}-38$, topotecan \\
\hline \multirow{5}{*}{ GSH conjugates } & Doxorubicin-SG \\
\hline & Cyclophosphamide-SG \\
\hline & Melphalan-SG \\
\hline & Leukotriene $\mathrm{C}_{4}$ \\
\hline & $\mathrm{PGA}_{2}-\mathrm{SG}, \mathrm{PGJ}_{2}-\mathrm{SG}$ \\
\hline \multirow[t]{4}{*}{ Glucuronide conjugates } & Etoposide-Gluc \\
\hline & SN-38-Gluc \\
\hline & $\mathrm{E}_{2} 17 \beta \mathrm{G}$ \\
\hline & Glucuronosylbilirubin \\
\hline \multirow[t]{2}{*}{ Sulfate conjugates } & Estrone-3-sulfate \\
\hline & Dehydroepiandrosterone sulfate \\
\hline
\end{tabular}

Source: Deeley RG and Cole SP. Substrate recognition and transport by multidrug resistance protein 1 (ABCC1), FEBS Lett, 580: 1103-1111, 2006. 
to examine the contribution of each gene to basal drug resistance to a panel of drugs including topotecan and SN-38 (101). In Mdr1a/1b null MEFs, a small (but significant) increase was observed in sensitivity to topotecan and SN-38. However, a much larger increase in topotecan and SN-38 sensitivity was observed in the triple knockout MEFs (Mrp1 and Mdr1a/1b null). Therefore, the camptothecin analogs are better substrates for MRP1 than Pgp.

\subsubsection{MRP1 expression in neuroblastoma}

Because MRP1 transports multiple drugs used in neuroblastoma therapy including the camptothecin analogs, the relationship between MRP1 expression in neuroblastoma and outcome has been examined. Norris and colleagues used RT-PCR to determine the MRP1 mRNA expression in 60 primary, untreated neuroblastomas (10). Patients who received chemotherapy were treated with regimens including the MRP1 substrates vincristine and doxorubicin. The cumulative survival rate was higher in patients with low MRP1 expression than in those with high expression $(94 \%$ vs. 57\%, respectively, $P<0.001$, log-rank test). The 5-year event-free survival (EFS) rate was also higher in patients with low MRP1 expressing tumors than in those with high expression (46\% vs. $91 \%$, respectively).

The same group confirmed these findings in a large prospective study of 209 neuroblastoma samples using RT-PCR (12). When all patients were evaluated based upon MRP1 expression level, 5-year EFS was significantly higher in patients with low MRP1 expressing tumors in comparison to high $M R P 1$ expression $(76 \%$ vs. $40 \%, P<0.001, \log$ rank test). 
High MRP1 expression remained a significant predictor of outcome in three subsets of patients. First, in patients with localized disease, those with high MRP1 had worse EFS than those with low expression ( $56 \%$ vs. $90 \%, P=0.001$, log-rank test). Next, children with MYCN nonamplified tumors and high MRP1 had worse EFS than those with low expression ( $46 \%$ vs. $81 \%, P<0.001$, log-rank test). Finally, in children with stage 4 disease high MRP1 was associated with extremely poor EFS compared to those with low expression ( $14 \%$ vs. $44 \%, P<0.001$, log-rank test). Therefore, even in patients with favorable prognostic features such as localized disease or nonamplified MYCN, MRP1 expression may be a useful indicator of prognosis that can be used to guide therapy.

MRP1 mRNA expression is a prominent feature in neuroblastoma and may be a strong, independent indicator of poor prognosis. However, due to the essentially ubiquitous MRP1 expression in normal tissue, modulation of tumor-specific MRP1mediated resistance presents a significant challenge. However, the relationship between MRP1 expression in neuroblastoma and poor prognosis may not be solely attributed to drug efflux. As MRP1 expression is strongly correlated with MYCN amplification, the expression of this $\mathrm{ABC}$ transporter may be a surrogate marker for another currently unidentified MYCN target gene responsible for poor prognosis. Furthermore, these studies have focused on the MRP1 mRNA expression, and therefore the question remains about the mechanistic implications of the strong correlation between MYCN amplification and MRP1 mRNA expression. It has not yet been determined whether MRP1 and MYCN have independent roles in neuroblastoma. A mechanistic explanation for the poor prognosis in patients with high $M R P 1$ expression remains to be elucidated. 


\subsubsection{MRP2}

The human ABCC2 gene contains 32 exons (102) mapping to chromosome 10q24 and encodes a $180 \mathrm{kDa}$ protein with alternative nomenclature that includes cMOAT (for canalicular multispecific organic anion transporter) and MRP2 (multidrug resistance associated protein 2). The MRP2 protein sequence is $48 \%$ homologous to MRP1 (103). MRP2 is primarily expressed in the hepatocyte canalicular membrane and mediates the hepatobiliary excretion of organic anions including glutathione and bilirubin glucuronides (104). MRP2 expression has also been demonstrated at the apical membrane of the renal proximal tubules (105).

\subsubsection{MRP2 substrates}

As with Pgp and MRP1, there is considerable overlap in substrate specificity between MRP1 and MRP2. MRP2 substrates include vincristine, vinblastine, methotrexate, cisplatin, doxorubicin, epirubicin, etoposide, cyclophosphamide, irinotecan, and SN-38 $(106,107,108)$. MRP2 both transports and confers resistance to irinotecan and SN-38. First in the HepG2 hepatic cancer cell line expressing MRP2, antisense RNA to MRP2 reduced MRP2 expression and increased sensitivity to both irinotecan and SN-38 (106). Chu and colleagues used canalicular membrane vesicles isolated from rats to demonstrate the ATP-dependent transport of both the lactone and carboxylate forms of SN-38G and the carboxylate forms of irinotecan and SN-38 (109).

\subsubsection{MRP2 in neuroblastoma}

To date MRP2 protein expression has not been demonstrated in neuroblastoma. Norris and colleagues have demonstrated that MRP2 mRNA levels in patients with neuroblastoma did not discriminate between treatment success or failure, but analysis of 
MRP2 protein expression was not reported (110). However, due to MRP2-mediated transport of drugs used in neuroblastoma therapy including irinotecan/SN-38, vincristine, cisplatin, and doxorubicin, MRP2 protein expression should be further evaluated in neuroblastoma. Although neuroblastoma does not arise from a tissue that normally expresses MRP2, expression of MRP2 in neuroblastoma is possible. Due to the potential for efflux of irinotecan and SN-38 in MRP2-expressing tumor cells, if MRP2 is expressed in neuroblastoma, the relationship between MRP2 expression and response to therapy including MRP2 substrates should be explored.

\subsubsection{MRP4}

The human ABCC4 gene maps to chromosome 13q32 (111) and encodes the 170 $\mathrm{kDa}$ protein MRP4 (112). The organic anion transporter MRP4 was first identified in 1997 by searching the human expressed sequence tag (EST) database for sequences homologous to the 3'-ends of MRP1 and MRP2 (113). Human MRP4 mRNA expression was demonstrated in lung, kidney, bladder, and gall bladder and in drug resistant cell lines derived from several tumor types including non-small cell lung cancer, small cell lung cancer, lung adenocarcinoma, and promyelocytic leukemia. Tissue distribution studies have shown that MRP4 is also expressed in the adrenal gland, on the apical side of renal proximal tubules and capillaries in the brain, and the basolateral side of the choroid plexus epithelium $(114,115,116)$.

\subsubsection{MRP4 substrates}

MRP4 is an organic anion transporter, and its substrates include both xenobiotics and endogenous substances. MRP4 was identified as a transporter of the nucleoside 
analog 9-(2-phosphonylmethoxyethyl) adenine (PMEA) (111). Inverted membrane vesicles were used to identify the endogenous MRP4 substrates cGMP, cAMP, and estradiol 17- $\beta$-D-glucuronide $\left(\mathrm{E}_{2} 17 \beta \mathrm{G}\right)$ (117). Prostaglandin $\mathrm{E}_{1}\left(\mathrm{PGE}_{1}\right)$ and $\mathrm{PGE}_{2}$ have also been identified as endogenous substrates (118). MRP4 also effluxes 6mercaptopurine (6-MP) monophosphates, 6-thioguanine (6-TG) monophosphates, and methotrexate $(117,119)$ and confers resistance to cyclophosphamide, but not etoposide, 5fluorouracil, carboplatin, vincristine, vinblastine, or paclitaxel (120).

Topotecan is an MRP4 substrate (114). In particular, increasing topotecan concentrations reduced ATP-dependent uptake of $\mathrm{E}_{2} 17 \beta \mathrm{G}$ into inverted membrane vesicles overexpressing MRP4. Also, Saos2 cells overexpressing MRP4 were 5 times more resistant to topotecan and accumulated less topotecan intracellularly than the parental cell line.

Subsequently Tian and colleagues used stably transfected HepG2 (which endogenously expresses MRP4 (121)) cells overexpressing MRP4 to demonstrate that MRP4 confers resistance to other camptothecin analogs (120). Cells overexpressing MRP4 were 3- to 6-fold resistant to irinotecan lactone. MRP4 conferred 8- to 9-fold resistance to $\mathrm{SN}-38$ lactone and carboxylate. The level of resistance to $\mathrm{SN}-38$ and irinotecan was similar to that observed for the classical MRP4 substrates bis-POMPMEA and methotrexate. Furthermore, this increase in resistance to both SN-38 and irinotecan was accompanied by a 2- to 4-fold reduction in intracellular accumulation of both irinotecan and SN-38.

Using the same MRP4 overexpressing HepG2 cell line, Tian and colleagues also confirmed that MRP4 transports and confers resistance to topotecan (122). MRP4 
overexpression conferred 12-fold resistance to topotecan. The level of topotecan resistance in MRP4 overexpressing cells was similar to that for bis-POM-PMEA. In addition, topotecan intracellular accumulation was reduced in the MRP4 overexpressing cells. Due to the endogenous MRP4 expression in the HepG2 cell line, these data provide information about the contribution of overexpressed MRP4 to camptothecin analog transport and resistance. The data do not give information about the level of resistance conferred by the basal level of MRP4 expression. To determine the contribution of basal MRP4 expression, the gene could be silenced using RNAi. Alternatively, if an inhibitor specific for MRP4 (and not other ABC transporters) existed, it could be used to assess the contribution of MRP4 to camptothecin analog transport and resistance.

\subsubsection{MRP4 in neuroblastoma}

Norris and colleagues reported that MRP4 mRNA expression was correlated with MYCN amplification in neuroblastoma and was an independent predictor of poor outcome (110). Although MYCN has not yet been shown to activate MRP4 transcription, the MRP4 promoter contains an E box. Therefore, MRP4 may be a MYCN target gene. In addition, MRP4 may confer clinical resistance to drugs used in neuroblastoma therapy, notably the camptothecin analogs. Hence, the contribution of MRP4 expression to camptothecin analog resistance in neuroblastoma will be explored in this project.

\subsubsection{BCRP}

The breast cancer resistance protein (BCRP) is the product of the ABCG2 gene and maps to chromosome 4q22. BCRP aliases include MXR (for mitoxantrone resistance) and $\mathrm{ABCP}$ for (placental $\mathrm{ABC}$ transporter). $\mathrm{BCRP}$ was identified by Doyle 
and colleagues in MCF7AdrVp, a breast cancer cell line selected for doxorubicin resistance that did not express Pgp or MRP1 (123). At the same time Allikmets and colleagues demonstrated BCRP expression in the placenta (124). Shortly thereafter, Maliepaard and colleagues demonstrated BCRP overexpression in an ovarian cancer cell line selected for topotecan resistance (125). BCRP is comprised of 663 amino acids and a single NBD at the amino terminus. BCRP domains are organized $\mathrm{NH}_{2}-\mathrm{NBD}-\mathrm{TMD}$ $\mathrm{COOH}$, giving the appearance of a half-transporter in contrast to Pgp and MRP $\left(\mathrm{NH}_{2}\right.$ TMD-NBD1-TMD-NBD2-COOH) (123). However, BCRP homodimerization is required for function (126).In addition to expression in placental syncytiotrophoblasts, BCRP is also expressed in the apical membrane of the small intestine epithelium, bile canaliculi, and the apical membrane of endothelial cells in brain vasculature (127).

\subsubsection{BCRP substrates}

BCRP transports a variety of xenobiotics and endogenous substrates (Table 1.8). Specifically, several studies have demonstrated that BCRP has a role in camptothecin analog resistance. The majority of data addressing the substrate specificity of each transporter has been generated using drug selected cell lines. Reduced accumulation and increased resistance to topotecan and $\mathrm{SN}-38$ have been demonstrated in BCRP overexpressing cell lines selected for drug resistance $(125,128)$. Because this selection process can produce numerous genetic and post-translational alterations, the most reliable substrate specificity data is generated in isogenic systems. Houghton and colleagues demonstrated that Saos2 cells transfected with ABCG2 and overexpressing BCRP are 12fold and 50-fold more resistant to topotecan and $\mathrm{SN}-38$, respectively, than the vector control cell line (129). All of these studies taken together indicate that BCRP confers 
Table 1.8 Partial list of BCRP substrates.

\begin{tabular}{ll}
\hline Xenobiotic Substrates & Endogenous Substrates \\
\hline Mitoxantrone & $\mathrm{E}_{2} 17 \beta \mathrm{G}$ \\
Topotecan & Estrone-3-sulfate \\
Irinotecan, SN-38, SN-38 glucuronide & Dehydroepiandrosterone \\
$\begin{array}{l}\text { Methotrexate, methotrexate } \\
\text { polyglutamates }\end{array}$ & \\
\hline
\end{tabular}

Source: Litman $\mathrm{T}$, et al. The multidrug-resistant phenotype associated with overexpression of the new ABC half-transporter, MXR (ABCG2), J Cell Sci, 113 ( Pt 11): 2011-2021, 2000.

resistance to the camptothecin analogs SN-38 and topotecan.

\subsubsection{BCRP in neuroblastoma}

The role of BCRP expression in neuroblastoma has not been studied extensively. In one study of the mRNA expression of $\mathrm{ABC}$ transporters in neuroblastoma, BCRP mRNA was detected in neuroblastoma tumor specimens from patients, but the level of mRNA expression was not related to treatment outcome (110). The only other study of BCRP expression in neuroblastoma used immunohistochemistry to demonstrate BCRP protein expression in the xenograft NB1691 (130). Therefore, the extent to which BCRP is expressed in neuroblastoma tumors in children and its role in response to camptothecin analogs is unknown.

In summary, BCRP plays a role in camptothecin analog tumor resistance. Therefore, the contribution of BCRP to camptothecin analog resistance in neuroblastoma will be explored in this project. 


\subsubsection{Modifying ABC transporter function}

To determine the role of $\mathrm{ABC}$ transporters in drug resistance, the function and/ or expression of the transporter must be modulated. A small molecule chemical modulator can be used to inhibit transport or compete with substrates. Table 1.9 contains a partial list of small molecule chemical modulators of $\mathrm{ABC}$ transporters. However, no small molecule chemical inhibitor exists that inhibits a single $\mathrm{ABC}$ transporter. These chemical modulators of $\mathrm{ABC}$ transporters are therefore more useful in modulating the activity of a variety of transporters rather than in studying the contribution of a single transporter to resistance.

Other useful approaches to investigate the function of specific transporters in neuroblastoma cells or normal tissues include gene knockout, RNA interference (RNAi), and overexpression. Null models in which the gene for a specific $\mathrm{ABC}$ transporter has been knocked out can be used both in vitro and in vivo. For example, differences in drug sensitivity between knockout and wild type MEFs can be determined in vitro to determine the contribution of basal ABC transporter expression in normal tissues (101). Knockout mice can be compared to wild-type mice in pharmacokinetic studies. However, this approach is laborious and expensive. Moreover, it does not directly test the function of the human transporter in a human system such as a cell line derived from a human tumor sample. Also, due to the variation in sensitivity of different tumor types to cytotoxic drugs, the contribution of $\mathrm{ABC}$ transporters to drug resistance in a particular tumor type should be studied in the tumor or cell line of interest.

Exogenous overexpression of $\mathrm{ABC}$ transporters is another useful tool for studying $\mathrm{ABC}$ transporter function. This approach has been used consistently to identify 


\section{Table 1.9 Chemical modulators of ABC transporters.}

\begin{tabular}{lll}
\hline Drug & Transporters Inhibited & References \\
\hline Verapamil & Pgp, MRP1, MRP4 & $(18,131,132)$ \\
Quinidine & Pgp, MRP1, MRP2 & $(18)$ \\
Valspodar & Pgp, MRP1 & $(18)$ \\
MK571 & MRP1, MRP2, MRP4, & $(133,134,112,135)$ \\
& MRP7 & \\
Gefitinib & Pgp, BCRP & $(130,136)$ \\
GF120918 & Pgp, BCRP & $(137,138,139,140)$ \\
Indomethacin & MRP1, MRP4 & $(141,118,112)$ \\
\hline
\end{tabular}

Sources: Litman T, et al. From MDR to MXR: new understanding of multidrug resistance systems, their properties and clinical significance, Cell and Molecular Life Sciences, 58: 931-959, 2001.

Abe $\mathrm{T}$, et al. Chemosensitisation of spontaneous multidrug resistance by a 1,4dihydropyridine analogue and verapamil in human glioma cell lines overexpressing MRP or MDR1, Br J Cancer, 72: 418-423, 1995.

Bai J, et al. Multidrug resistance protein 4 (MRP4/ABCC4) mediates efflux of bimaneglutathione, Int J Biochem Cell Biol, 36: 247-257, 2004.

Leier I, et al. The MRP gene encodes an ATP-dependent export pump for leukotriene C4 and structurally related conjugates, J Biol Chem, 269: 27807-27810, 1994.

Leier I, et al. ATP-dependent para-aminohippurate transport by apical multidrug resistance protein MRP2, Kidney Int, 57: 1636-1642, 2000.

Jedlitschky G, et al. The nucleotide transporter MRP4 (ABCC4) is highly expressed in human platelets and present in dense granules, indicating a role in mediator storage, Blood, 104: 3603-3610, 2004.

Chen ZS, et al. Characterization of the transport properties of human multidrug resistance protein 7 (MRP7, ABCC10), Mol Pharmacol, 63: 351-358, 2003.

Stewart CF, et al. Gefitinib enhances the antitumor activity and oral bioavailability of irinotecan in mice, Cancer Res, 64: 7491-7499, 2004.

Leggas $M$, et al. Gefitinib modulates the function of multiple ATP-binding cassette transporters in vivo, Cancer Res, 66: 4802-4807, 2006.

Hyafil F, et al. In vitro and in vivo reversal of multidrug resistance by GF120918, an acridonecarboxamide derivative, Cancer Res, 53: 4595-4602, 1993. 


\section{Table 1.9 (continued)}

Allen JD, et al. Potent and specific inhibition of the breast cancer resistance protein multidrug transporter in vitro and in mouse intestine by a novel analogue of fumitremorgin C, Mol Cancer Ther, 1: 417-425, 2002.

Maliepaard M, et al. Circumvention of breast cancer resistance protein (BCRP)-mediated resistance to camptothecins in vitro using non-substrate drugs or the BCRP inhibitor GF120918, Clin Cancer Res, 7: 935-941, 2001.

Allen JD, et al. The mouse Bcrp1/Mxr/Abcp gene: amplification and overexpression in cell lines selected for resistance to topotecan, mitoxantrone, or doxorubicin, Cancer Res, 59: 4237-4241, 1999.

Decleves X, et al. Molecular and functional MDR1-Pgp and MRPs expression in human glioblastoma multiforme cell lines, Int J Cancer, 98: 173-180, 2002.

Reid G, et al. The human multidrug resistance protein MRP4 functions as a prostaglandin efflux transporter and is inhibited by nonsteroidal antiinflammatory drugs, Proc Natl Acad Sci U S A, 100: 9244-9249, 2003.

Jedlitschky G, et al. The nucleotide transporter MRP4 (ABCC4) is highly expressed in human platelets and present in dense granules, indicating a role in mediator storage, Blood, 104: 3603-3610, 2004. 
endogenous and xenobiotic substrates and to demonstrate multi-drug resistance. Cell lines overexpressing $\mathrm{ABC}$ transporters have the advantage over those with basal expression levels in that differences in substrate accumulation or resistance are more pronounced and easily detected. However, due to protein abundance this approach does not give much information about the role of normal, basal protein expression.

RNA interference (RNAi) can be used to silence the expression of a specific gene. Just as for targeting the disruption of a particular gene locus to knock out a gene, RNAi can be used to study the contribution of basal gene expression to the phenotype of interest.

RNAi was first described as double-stranded RNA (dsRNA) mediated sequencespecific post transcriptional gene silencing in the nematode Caenorhabditis elegans (142). The highly conserved RNAi phenomenon of gene silencing has been observed in protozoa, invertebrates, vertebrates, plants, fungi, and algae. RNAi is hypothesized to serve as an antiviral defense mechanism $(143,144)$.

Experimentally, RNAi can be used to reduce or eliminate the expression of a protein by targeting its mRNA for degradation. Dicer, an RNase III-like enzyme cleaves long dsRNA molecules into 21- to 23-nucleotide small interfering RNAs (siRNAs) (145). Subsequently the siRNAs are incorporated into the RNAi-induced silencing complex (RISC). ATP activates the RISC complex to expose the antisense siRNA strand and bind the targeted complementary mRNA (146). Finally, the mRNA is degraded by exonucleases $(147,148)$. Exogenously delivered RNAi used to inhibit ABC transporter function has an advantage over chemical inhibition due to specific targeting of a single gene. 
Despite the ability to achieve targeted silencing of a single gene based upon nucleotide sequence complementarity, RNAi can induce nonspecific off-target effects that include the interferon response and concentration-dependent induction of off-target genes(149). The interferon response elicited by introduction of long dsDNA can be circumvented by introduction of siRNAs, which are short dsDNA molecules 21-22 bp in length that specifically target degradation of mammalian genes. Using the minimum effective siRNA concentration eliminates the induction of off-target or "siRNAresponse" genes (150).

To achieve gene silencing with RNAi, the construct must be appropriately delivered to the target cell and can be transient or stable. RNAi can be delivered as small interfering RNA (siRNA) or short hairpin RNA (shRNA) oligonucleotides to achieve transient knockdown of a gene. Plasmids or retroviral vectors containing a shRNA cassette under the control of the RNA polymerase promoter (i.e., H1 or U6) can be used to achieve stable expression of RNAi. Brummelkamp and colleagues achieved stable RNAi by including a puromycin resistance cassette in a plasmid containing the $\mathrm{H} 1$ promoter and a 19-nt gene specific insert followed by a spacer to form a hairpin and then the reverse complement of the 19-nt sequence (151). Therefore, stable retroviral mediated RNAi will be used to silence $\mathrm{ABC}$ transporter expression in neuroblastoma cell lines to determine the contribution of basal expression to in vitro and in vivo camptothecin analog sensitivity.

\subsection{Summary}

High-risk neuroblastoma remains a significant clinical challenge despite aggressive and intense therapy. The camptothecin analogs topotecan and irinotecan are 
useful components of neuroblastoma therapy. Despite a favorable anti-tumor response rate to pharmacokinetically guided topotecan in children with high-risk neuroblastoma, some patients failed to achieve a clinically meaningful response. Therefore, we postulate that molecular mechanisms of drug resistance were also present in primary neuroblastoma cells. Specifically we propose that Pgp, MRP1, MRP2, MRP4, and BCRP may be expressed in neuroblastoma and may confer resistance to topotecan and irinotecan/ SN-38. The primary hypothesis of this dissertation was that the expression of $\mathrm{ABC}$ transporters contributes to clinical resistance of neuroblastoma to the cytotoxicity of topotecan and irinotecan/ SN-38.

\subsection{Specific aims}

The primary objective of this work was to understand the role of $\mathrm{ABC}$ transporters in neuroblastoma resistance to the camptothecin analogs. Potentially relevant ABC transporters include Pgp, MRP1, MRP2, MRP4, and BCRP. To accomplish this objective we will use genetically modified neuroblastoma cell lines, xenograft models, and biopsy specimens from children with high-risk neuroblastoma.

\subsubsection{To determine the contribution of $A B C$ transporters to topotecan and irinotecan sensitivity in neuroblastoma cell lines}

A panel of neuroblastoma cell lines representing a range of topotecan and irinotecan sensitivities will be evaluated to determine if differential expression of $\mathrm{ABC}$ transporters identifies specific transporters that may confer clinical resistance to the camptothecin analogs. RNAi will be used to silence MRP4 and Pgp in a resistant 
neuroblastoma cell line. In addition, MRP4 will be overexpressed in a sensitive neuroblastoma cell line.

\subsubsection{To determine the role of MRP4 in neuroblastoma xenograft response to topotecan}

A murine xenograft model in which MRP4 has been silenced via RNAi will be established. Mice bearing neuroblastoma xenografts with silenced MRP4 will be treated with topotecan to assess the contribution of basal MRP4 expression to topotecan resistance in neuroblastoma in vivo.

\subsubsection{To determine the role of $A B C$ transporter expression in response to topotecan in children with high-risk neuroblastoma} Immunohistochemistry will be used to characterize the $\mathrm{ABC}$ transporter expression in neuroblastoma biopsy specimens from children with high-risk disease. The association between $\mathrm{ABC}$ transporter expression and response to pharmacokinetically guided topotecan will be evaluated.

In summary, a subset of children with high-risk neuroblastoma did not respond to camptothecin analog based therapy. ABC transporters (e.g., MRP4 and Pgp) may contribute to this clinical resistance. These in vitro, in vivo, and clinical studies of neuroblastoma will help to define the role of MRP4 and Pgp in camptothecin analog resistance in neuroblastoma. 


\section{Chapter 2. MRP4 and P-glycoprotein confer resistance to topotecan in neuroblastoma}

\subsection{Introduction}

Neuroblastoma is the most common extracranial solid tumor in children. The prognosis for children diagnosed with high-risk neuroblastoma is poor, with 5-year survival less than $30 \%(152)$. Topotecan and irinotecan anti-tumor activity in neuroblastoma has been demonstrated in preclinical studies and phase I clinical trials, including studies in children with high-risk disease $(64,66,70,71,17,76,153)$. Furthermore, there is a steep topotecan systemic exposure/ anti-tumor response relationship. However, substantial interindividual variation in topotecan pharmacokinetics exists in children. Topotecan systemic clearance, the determinant of systemic exposure as measured by AUC, varies at least ten-fold in children (49). Therefore, dosing topotecan based solely on body surface area may lead to some antitumor responses but also a range of toxic and subtherapeutic exposures.

Minimizing interindividual variability in topotecan systemic exposure with a pharmacokinetically guided dosing approach has been successful in terms of achieving the putatively effective systemic exposure in children with high-risk neuroblastoma (15). In a phase II trial this pharmacokinetically guided dosing approach also resulted in very good anti-tumor activity. Out of 28 children with high-risk neuroblastoma evaluable for a response to pharmacokinetically guided topotecan, 1 complete response and 17 partial responses were observed. However, the ten children with tumors that failed to respond may represent a subset of the disease resistant to topotecan $(49,154,155)$. 
Although clinical resistance to topotecan is multi-factorial, $\mathrm{ABC}$ transporters have been implicated in multi-drug resistance in neuroblastoma. Pgp and MRP1 have been associated with diminished response of neuroblastoma to chemotherapy $(52,156)$. Many of the drugs used to treat neuroblastoma are MRP1 and/ or Pgp substrates, including doxorubicin, vincristine, and etoposide. However, mechanistic studies validating a link between protein expression of $\mathrm{ABC}$ transporters and resistance to chemotherapeutic agents are lacking. Moreover, in neuroblastoma the expression of other members of the $\mathrm{ABC}$ transporter superfamily such as MRP2, MRP4, and BCRP has not been well characterized.

We have previously demonstrated that topotecan is an MRP4 substrate (114). Topotecan, irinotecan, and SN-38 are BCRP substrates. Irinotecan and $\mathrm{SN}-38$ are also MRP2 substrates $(125,157,158)$. Several investigators have demonstrated that Pgp confers resistance to topotecan. In this study we used RNAi to reduce MRP4 and Pgp expression in neuroblastoma cell lines to evaluate the effect of $A B C$ transporters on topotecan and irinotecan resistance.

RNAi can be mediated through siRNA or shRNA. Reductions in protein expression mediated by siRNA are transient. In cell culture, this gene silencing persists for 3-5 days (159). Variation in transfection efficiency also limits the application of transient transfection of siRNA. Knock down via transient transfection of siRNA targeting $\mathrm{ABC}$ transporters is suitable to test certain phenotypes such as drug accumulation. However, this transient system is not suitable to test longer time-dependent phenotypes such as drug sensitivity using the MTT assay. 
In contrast with transient siRNA-mediated downregulation of gene expression, retroviral vector mediated shRNA can persist for long periods of time, due to integration in the host genome. In addition, retroviral vectors can carry selection markers such as puromycin resistance genes or green fluorescent protein (GFP). Continuous selection pressure can be applied by culturing in the presence of puromycin. Alternatively, drug interactions or non-specific effects of selection antibiotics can be avoided using GFP as a selection marker. GFP expressing clones can be sorted by FACS, and GFP expression over time can be monitored via flow cytometry or fluorescence microscopy. But in all cases, reduced protein expression of the shRNA targeted gene must be confirmed.

Thus, the objectives of the present study were to determine whether MRP4 and Pgp confer resistance to topotecan and SN-38 in neuroblastoma. Therefore, we used shRNA to knock down MRP4 and Pgp expression in the cell line NB1691. Furthermore, we overexpressed MRP4 in the cell line NB1643. These in vitro models were used to evaluate intracellular drug accumulation and sensitivity to camptothecin analogs.

\subsection{Materials and methods}

\subsubsection{Chemicals and cell lines}

Topotecan, ${ }^{3} \mathrm{H}$-topotecan, and ${ }^{14} \mathrm{C}$-topotecan were generously provided by GlaxoSmithKline (King of Prussia, PA). SN-38, the active component of the pro-drug irinotecan (CPT-11) was provided by Pfizer (New York, NY).

NB1691, NB1643, SKNAS, SKNSH, NBEB (also known as SJNB1), and NBSD (also known as SJNB4) cell lines were kindly provided by Dr. Peter Houghton (St. Jude Children's Research Hospital, Memphis, TN). Saos2 pcDNA and Saos2 MRP4 cell lines 
were kindly provided by Dr. John Schuetz (St. Jude Children's Research Hospital). The amphotropic retroviral packaging cell line Phoenix was obtained from ATCC (Manassas, VA). Cells were maintained in RPMI 1640 (Mediatech, Herndon, VA), 10\% FBS (HyClone, Logan, UT), and 1\% L-glutamine (Mediatech) at $37^{\circ} \mathrm{C}, 5 \% \mathrm{CO}_{2}$.

\subsubsection{Transient MDR1 siRNA}

Three siRNA constructs targeting MDR1 (Ambion, Austin, TX) were transfected (30 nM) into NB1691 using siPORT Amine (Ambion). The sequence targeting MDR1 exon 4 was selected for further use in stable shRNA studies, and the primers 5'-GATCCCCGGGAAAAGAAACCAACTGTCTCAAGAGGACAGTTGGTTTCTTTTCCCTTTTTGGAAA-3' and 5'-AGCTTTTCCAAAAAGGGAAAAGAAACCAACTGTCCTCTTGAGACAGTTGGTTTCTTTTCCCGGG-3' containing BglII and HindIII half-sites were cloned into pSuper.

\subsubsection{Stable RNAi by cloning MRP4 or MDR1 shRNA into MSCVGFP}

The pSUPER42 (118) vector containing a MRP4 shRNA cassette was generously provided by Dr. Piet Borst (Netherlands Cancer Institute, Amsterdam, Netherlands). The MSCVGFP42 construct was made by cloning a SmaI and SalI fragment from pSUPER42 containing the H1 promoter and MRP4 shRNA sequence into SalI and HindIII sites of MSCVGFP (160). The MSCV MDR1 shRNA GFP construct was made by cloning a SmaI and SalI fragment from pSuperMDR1shRNA into the SalI and HindIII sites of MSCVGFP. FuGENE6 (Roche Applied Science) was used to transfect MSCVGFP42, MSCV MDR1 shRNA GFP, or MSCVGFP into Phoenix producer cells. Retrovirus was harvested from Phoenix cells sorted for GFP expression, and NB1691 cells were 
transduced with vector alone, the MRP4 knockdown construct MSCVGFP42, or the Pgp knockdown construct MSCV MDR1 shRNA GFP. Clones with stable MRP4 or Pgp knockdown were generated by sorting single NB1691 cells expressing GFP into 96-well plates, expanding, and screening by real-time RT-PCR and Western blot for MRP4 or Pgp expression.

\subsubsection{Cloning MRP4 into NB1643}

The MRP4 expression vector MSCV MRP4 IRES GFP containing an internal ribosomal entry site permitting co-expression of MRP4 and GFP (kindly provided by Dr. John Schuetz) was transfected into Phoenix cells (161). NB1643 cells were transduced with retrovirus harvested from cells sorted for GFP expression. NB1643 cells overexpressing MRP4 were selected by FACS, expanded, and screened by Western blot for MRP4 expression.

\subsubsection{Real-time RT-PCR}

RNA was extracted from cells with Tri-Reagent (Molecular Research Center, Cincinnati, $\mathrm{OH}$ ), and cDNA was synthesized using a mixture of oligo-dT and random hexamer primers with the QuantiTect Reverse Transcription Kit (Qiagen, Valencia, CA). Real-time PCR analysis was conducted using gene specific primers (Table 2.1) and QuantiTect SYBR Green PCR Kit (Qiagen) on the ABI Prism 7900HT (Applied Biosystems, Foster City, CA). Thermal cycling conditions were as follows: 15 min at $95^{\circ} \mathrm{C}$ followed by 40 cycles of $95^{\circ} \mathrm{C}$ for $30 \mathrm{~s}, 60^{\circ} \mathrm{C}$ for $30 \mathrm{~s}, 72^{\circ} \mathrm{C}$ for $30 \mathrm{~s}$, and finally a denaturation stage. The mRNA expression level was determined by the comparative $C_{t}$ method. Fold-change in mRNA expression level was calculated by evaluating the 
Table 2.1 Primer sequences for real-time RT-PCR.

\begin{tabular}{llll}
\hline Gene & Primer Sense & Primer Antisense & Reference \\
\hline MDR1 & CTGTATTGTTTGCCACCACGA & AGGGTGTCAAATTTATGAGGCAGT & $(162)$ \\
MRP4 & CAGTACCTCAAAGCTGCAAGTC & CCCAGTATGAAAGCCACCAA & $(163)$ \\
BCRP & CAGGAGGCCTTGGGATACTT & TGCCACAGCAGTGGAATCT & $(164)$ \\
& & & \\
GAPDH & ACCACAGTCCATGCCATCAC & TCCACCACCCTGTTGCTGTA & $(163)$ \\
& & & \\
$\beta$-actin & GAGCACAGAGCCTCGCCTTT & TGACCCATGCCCACCATCAC & \\
\hline
\end{tabular}

Sources: Wunderlich K, et al. Vasospastic persons exhibit differential expression of ABC-transport proteins, Mol Vis, 9: 756-761, 2003.

Assem M, et al. Interactions between hepatic Mrp4 and Sult2a as revealed by the constitutive androstane receptor and Mrp4 knockout mice, J Biol Chem, 279: 2225022257, 2004.

Zamber CP, et al. Natural allelic variants of breast cancer resistance protein (BCRP) and their relationship to BCRP expression in human intestine, Pharmacogenetics, 13: 19-28, 2003. 
expression $2^{-\Delta \Delta \mathrm{Ct}}$ where $\mathrm{C}_{\mathrm{t}}$ is the cycle threshold, $\Delta \mathrm{C}_{\mathrm{t}}$ is the difference in mean cycle threshold between the gene of interest and the normalizing gene (e.g., $\mathrm{C}_{\mathrm{t} \text { MRP4 }}-\mathrm{C}_{\mathrm{t}} \mathrm{GAPDH}$ ), and $\Delta \Delta \mathrm{Ct}$ is the difference between the $\Delta \mathrm{Ct}$ 's of the reference and test samples (e.g., $\Delta \mathrm{C}_{\mathrm{t}}$ $\left.\mathrm{NB} 1691-\Delta \mathrm{C}_{\mathrm{tNB} 1691} 4.27\right)$.

\subsubsection{Western blot}

Membrane enriched fractions were isolated from cells by sonicating in homogenizing buffer $(100 \mathrm{mM}$ Tris- $\mathrm{HCl}, 100 \mathrm{mM} \mathrm{KCl}, 1 \mathrm{mM}$ EDTA, and protease inhibitors, Roche Applied Science, Indianapolis, IN) and centrifugation at 18,000 x $g$ for $15 \mathrm{~min}$. The pellet was resuspended in microsome storage buffer $\left(100 \mathrm{mM} \mathrm{KH_{2 }} \mathrm{PO}_{4}, 1\right.$ mM EDTA, 20\% glycerol, and protease inhibitors), and DNA was sheared by passing through a 27 gauge needle. In some cases, membrane proteins were isolated using the Mem-PER Eukaryotic Membrane Protein Extraction Kit (Pierce, Rockford, IL). Detergents used in the Mem-PER protein isolation were removed using the PAGEprep Advance Clean-Up Kit (Pierce).

Protein was quantitated by Bradford Assay (Bio-Rad, Hercules, CA). Proteins (10 $\mu \mathrm{g}$ per lane) were separated by SDS-PAGE (4-12\% Bis-Tris gel, MOPS running buffer, Invitrogen, Carlsbad, CA) and transferred to PVDF membranes. Blots were probed with the polyclonal rabbit-anti-human MRP4, kindly provided by Dr. John Schuetz, or the monoclonal antibodies $\mathrm{M}_{4} \mathrm{I}-10$ (rat anti-human and mouse MRP4, Axxora, San Diego, CA), BXP-21 (mouse anti-human BCRP, Kamiya Biomedical, Seattle, WA), AC-15 (mouse anti-human $\beta$-actin, Sigma, St. Louis, MO), and JSB-1 (mouse anti-human Pgp, Signet Labs, Dedham, MA). Proteins were visualized with ECL Plus (Amersham Biosciences, Piscataway, NJ). 
Membrane samples that appeared to express MRP4 with a higher molecular weight than the positive control were treated with the enzyme PNGaseF (New England Biolabs, Ipswich, MA) to deglycosylate proteins. For this reaction, $10 \mu \mathrm{g}$ of membrane protein were denatured in the reaction buffer for $10 \mathrm{~min}$ at $37^{\circ} \mathrm{C}$. Then 500 units PNGaseF was added for a $1 \mathrm{hr}$ incubation at $37^{\circ} \mathrm{C}$.

\subsubsection{Intracellular topotecan, PMEA, rhodamine 123, and mitoxantrone accumulation}

Neuroblastoma cells were plated in 6-well plates $(300,000$ cells/well) in media without phenol red and allowed to attach overnight. ${ }^{3} \mathrm{H}$-topotecan or ${ }^{14} \mathrm{C}$-topotecan was added (final concentration of $10 \mu \mathrm{M},{ }^{3} \mathrm{H}$-topotecan specific activity $100 \mathrm{dpm} / \mathrm{pmol}$ and ${ }^{14} \mathrm{C}$-topotecan specific activity $30 \mathrm{dpm} / \mathrm{pmol}$ ) for $6 \mathrm{hr}$ at $37^{\circ} \mathrm{C}$. MRP4 function was assessed by PMEA intracellular accumulation. Bis(POM) ${ }^{3} \mathrm{H}-\mathrm{PMEA}$ (Moravek Biochemicals, Brea, CA) was added $(10 \mu \mathrm{M})$ and incubated at $37^{\circ} \mathrm{C}$ for $20 \mathrm{hr}$. The media was removed, and cells were washed once in ice-cold PBS. Cells were detached by addition of $0.5 \mathrm{~N} \mathrm{NaOH}$ and acidified with $1 \mathrm{~N} \mathrm{HCl}$. Intracellular ${ }^{3} \mathrm{H}$-topotecan, ${ }^{14} \mathrm{C}$ topotecan, or ${ }^{3} \mathrm{H}-\mathrm{PMEA}$ was measured by scintillation counting with normalization to total protein content.

Pgp function was assessed by intracellular rhodamine accumulation in $10^{6}$ neuroblastoma cells incubated for 30 min with $0.1 \mathrm{mg} / \mathrm{mL}$ rhodamine 123 (165). Intracellular fluorescence was determined by FACS. Pgp function in cells expressing GFP, which fluoresces in the same channel as rhodamine 123, was assessed by 
intracellular ${ }^{3} \mathrm{H}$-mitoxantrone (Moravek Biochemicals) accumulation, as described for radiolabeled topotecan and PMEA.

\subsubsection{MTT assay}

Cells were plated in RPMI 1640 without phenol red, 10\% FBS, and 1\% Lglutamine in 96-well plates $(10,000$ cells/well) and allowed to attach overnight. Cells were exposed to various concentrations of topotecan $(1 \mathrm{nM}$ to $200 \mu \mathrm{M})$ or SN-38 $(1 \mathrm{nM}$ to $100 \mu \mathrm{M}$ ) for $6 \mathrm{hr}$, and the media was replaced. Viability was assessed 4 days later by the addition of MTT (Sigma, St. Louis, MO) for $3 \mathrm{hr}$. Formazan crystals produced only by viable cells were solubilized with $0.04 \mathrm{~N} \mathrm{HCl}$ in isopropanol. Absorbance was measured at $570 \mathrm{~nm}$ with wavelength correction at $650 \mathrm{~nm}$ on the $\mu$ Quant Microplate Spectrophotometer (Bio-Tek Instruments, Winooski, VT).

\subsubsection{Statistical considerations}

The drug concentration that inhibited $50 \%$ of cell growth $\left(\mathrm{IC}_{50}\right)$ was calculated according to the Michaelis-Menten equation. Student's t-test with Bonferroni correction was used to determine differences in $\mathrm{IC}_{50}$ between control and transduced neuroblastoma cell lines. Dunnett's multiple comparison tests was used to determine differences in intracellular drug accumulation between control and transduced neuroblastoma cell lines. All tests were two-tailed. 


\subsection{Results}

\subsubsection{Functional MRP4 and Pgp expression in neuroblastoma cell lines resistant to camptothecin analogs}

Since expression of $\mathrm{ABC}$ transporters confers resistance to topotecan and irinotecan/SN38, we evaluated the expression of Pgp, MRP1, MRP2, MRP4, and BCRP in six neuroblastoma cell lines. We determined that MRP1 was expressed in all neuroblastoma cell lines representing a spectrum of sensitivity to topotecan. In contrast, neither MRP2 nor BCRP was expressed in vitro in these six neuroblastoma cell lines. Next, we determined that both MRP4 and Pgp were differentially expressed in the six neuroblastoma cell lines (Figure 2.1). Both MRP4 and Pgp were expressed in NB1691. However, neither MRP4 nor Pgp were expressed in NB1643. SKNAS also expressed MRP4 but not Pgp. SKNSH expressed Pgp but not MRP4.

We also measured intracellular accumulation of the MRP4 substrate PMEA (Figure 2.2A). NB1691 and SKNAS, the two cells lines that expressed MRP4, exhibited the lowest intracellular PMEA accumulation, consistent with the hypothesis that MRP4 was functionally active and effluxing PMEA. In contrast, NB1643 accumulated the highest level of intracellular PMEA.

Likewise, to demonstrate functional expression of Pgp, we measured intracellular accumulation of the Pgp substrate rhodamine 123 (Figure 2.2B). The two Pgp expressing cell lines NB1691 and SKNSH accumulated the least rhodamine intracellularly, suggesting the presence of a functionally active efflux transporter. The highest rhodamine accumulation was observed in NB1643, consistent with the lack of an efflux transporter. 


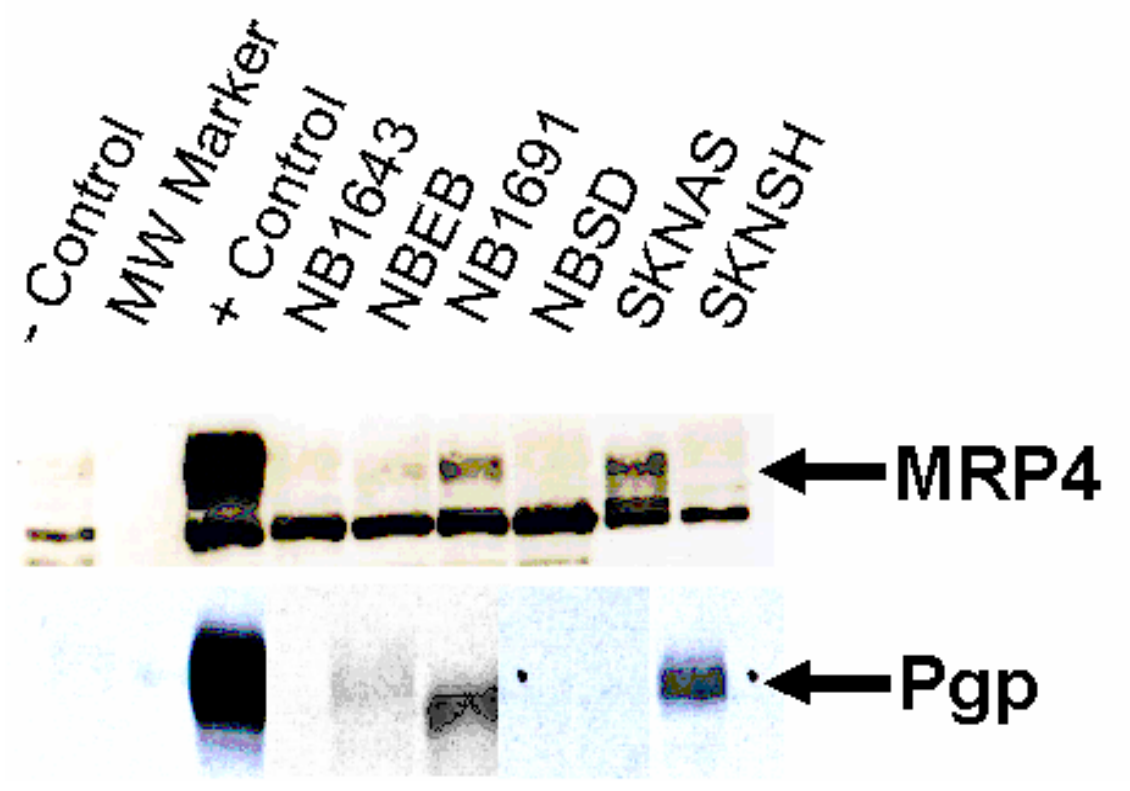

Figure 2.1 MRP4 and Pgp expression in topotecan resistant neuroblastoma cell lines.

The blot was probed with polyclone rabbit-anti-human MRP4 antibody. The negative and positive controls are from Saos2 pcDNA and Saos2 MRP4 cell lysates, respectively. MRP4 is expressed in NB1691 and SKNAS. The lower molecular weight band present in all samples is a non-specific band. Pgp is expressed in NB1691 and SKNSH. NB1643 expresses neither MRP4 nor Pgp. 
A

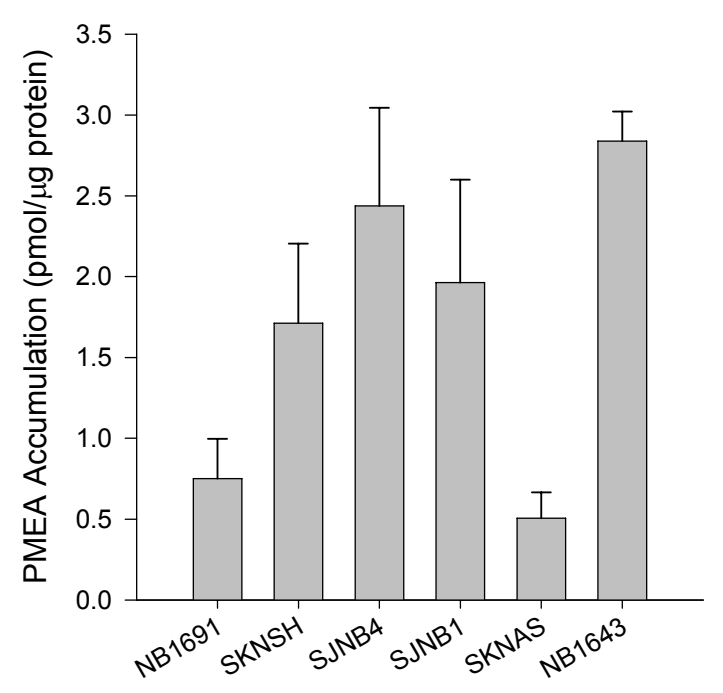

B

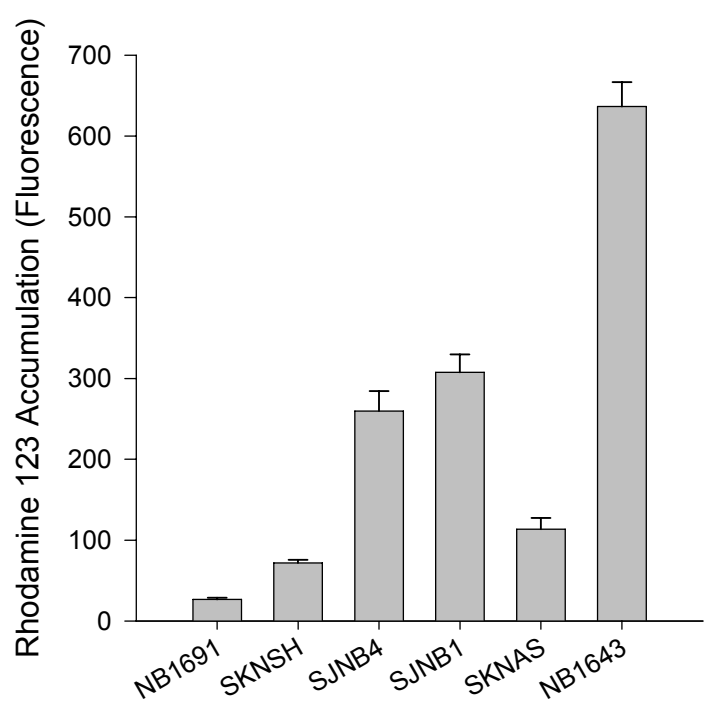

Figure 2.2 Accumulation of the MRP4 and Pgp substrates PMEA and rhodamine 123 in neuroblastoma cell lines.

PMEA accumulation is the lowest in the MRP4 expressing cell lines in NB1691 and SKNAS. The highest PMEA accumulation was observed in NB1643, which does not express MRP4 (A). Pgp expression in NB1691 and SKNSH leads to reduced intracellular accumulation of rhodamine 123. The highest rhodamine accumulation occurs in NB1643, which lacks Pgp expression (B). 
To evaluate whether the expression of $\mathrm{ABC}$ transporters was related to topotecan sensitivity in this panel of six neuroblastoma cell lines, we determined the $\mathrm{IC}_{50}$ to a six $\mathrm{hr}$ topotecan exposure by MTT assay. The topotecan $\mathrm{IC}_{50}$ was $8 \mu \mathrm{M}$ for NB1691 and 0.4 $\mu \mathrm{M}$ for NB1643 (Figure 2.3A). This 20-fold increased topotecan resistance in NB1691 is accompanied by reduced topotecan intracellular accumulation (Figure 2.3B) in NB1691 compared to NB1643, consistent with drug efflux as a resistance mechanism in NB1691. The next highest topotecan $\mathrm{IC}_{50}$ was observed in $\mathrm{SKNSH}$, which expressed Pgp but not MPR4.

\subsubsection{Functional MRP4 knockdown with stably expressed shRNA}

Due to MRP4 and Pgp expression in the two neuroblastoma cell lines most resistant to topotecan, and a lack of MRP4 or Pgp expression in the neuroblastoma cell line most sensitive to topotecan, we took two approaches to determine the relative contribution of MPR4 or Pgp to resistance. First, we used RNAi to reduce the expression of either MRP4 or Pgp in NB1691 and determine if there was an increase in sensitivity to the camptothecin analogs. The second approach to determine the contribution of MRP4 to camptothecin analog sensitivity was to overexpress MRP4 in NB1643 and determine if there was an increase in resistance to camptothecin analogs.

Using retroviral mediated expression of MRP4 shRNA, both MRP4 protein and mRNA expression were reduced in NB1691 GFP4.2 clones 7 and 9 compared to both the parental and vector only cell lines (Figure 2.4, Figure 2.5). Functional knockdown of MRP4 was confirmed by increased PMEA accumulation in NB1691 4.27 and 4.29 (Figure 2.6). Functional knockdown of MRP4 resulted in increased sensitivity to topotecan 4-fold and 2-fold in clones 4.27 and 4.2 9, respectively (Figure 2.7). 

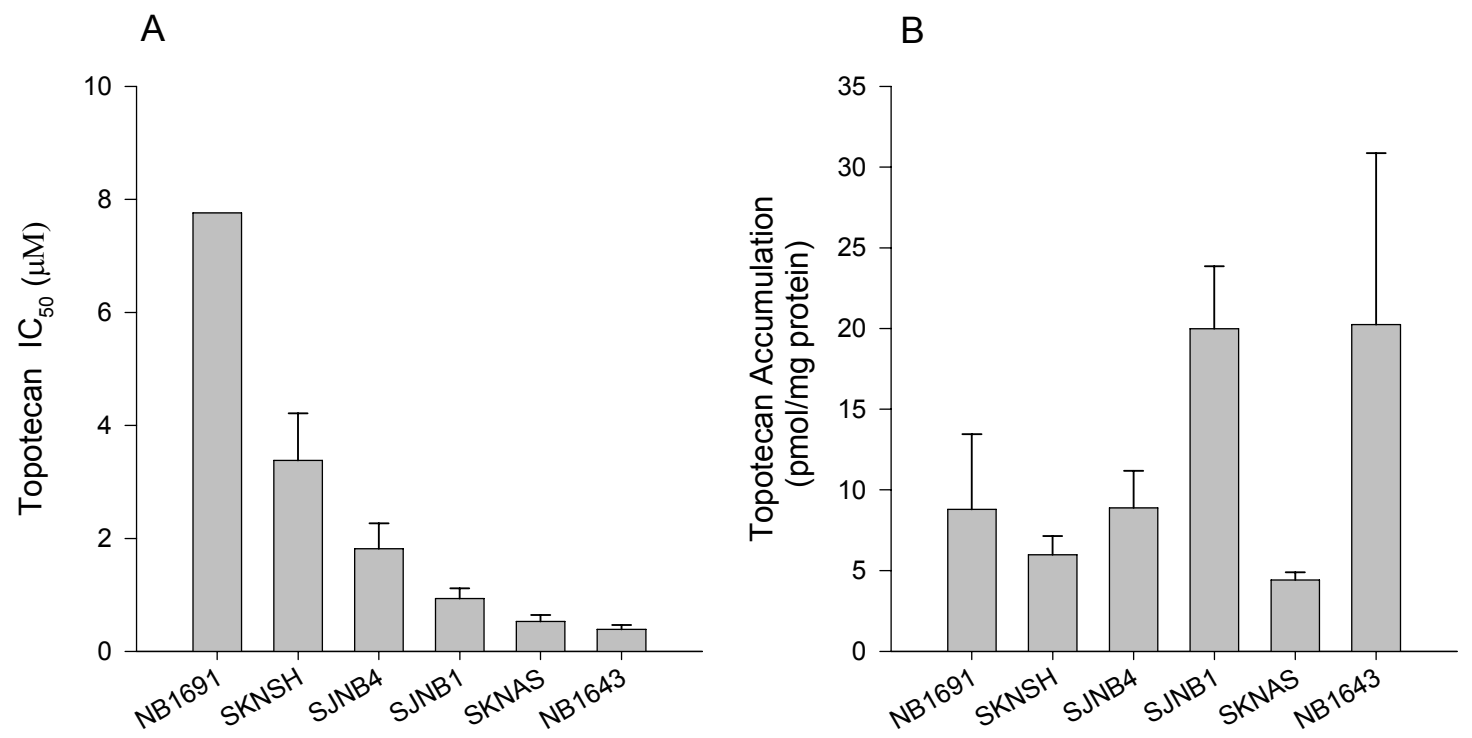

Figure 2.3 ${ }^{3} \mathrm{H}$-Topotecan sensitivity and accumulation in neuroblastoma cell lines.

The topotecan $\mathrm{IC}_{50}$ determined by MTT assay after a $6 \mathrm{hr}$ exposure to the drug is $8 \mu \mathrm{M}$ for NB1691 and $0.4 \mu \mathrm{M}$ for NB1643 (A). Less ${ }^{3} \mathrm{H}$-topotecan accumulates intracellularly in the resistant NB1691 compared NB1643 (B). 


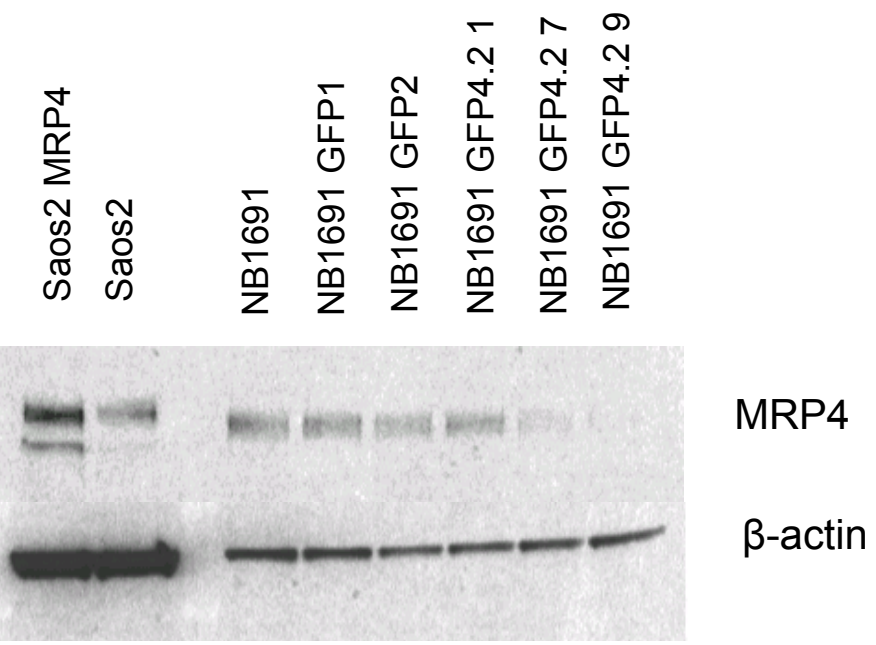

Figure 2.4 Immunoblot analysis of MRP4 expression in NB1691 cells transduced with MRP4 shRNA.

MRP4 expression was reduced in the NB1691 clones transduced with the retrovirus carrying the MRP4 shRNA and GFP cassettes. $\beta$-actin expression is depicted as the loading control. 


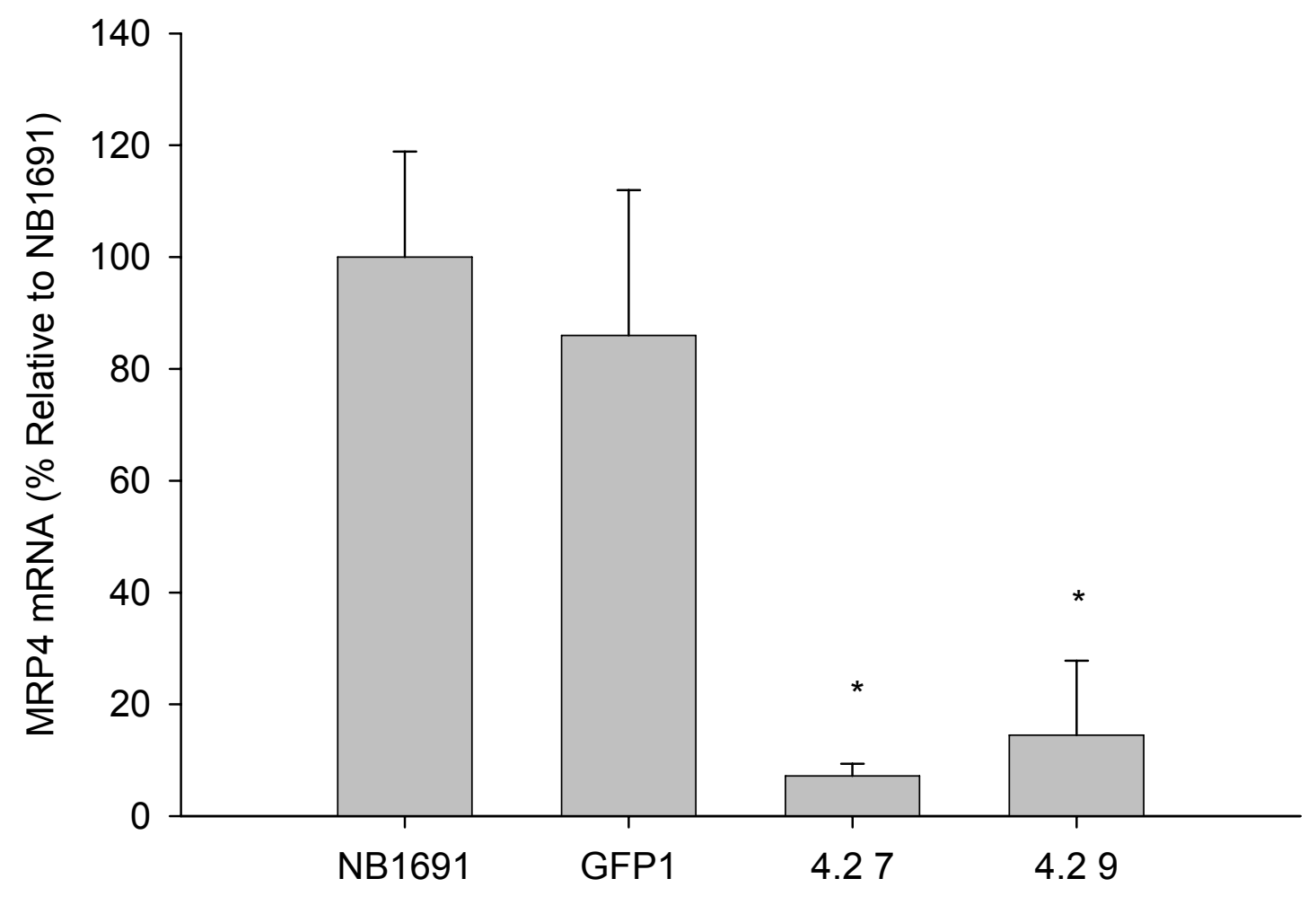

Figure 2.5 Real-time RT-PCR analysis in NB1691 MRP4 shRNA clones.

MRP4 mRNA expression is reduced in clones 4.27 and 4.29 compared to the parental cell line and vector control. Values are presented as mean \pm standard deviation.* $P \leq 0.01$ (t-test with Bonferroni correction). 


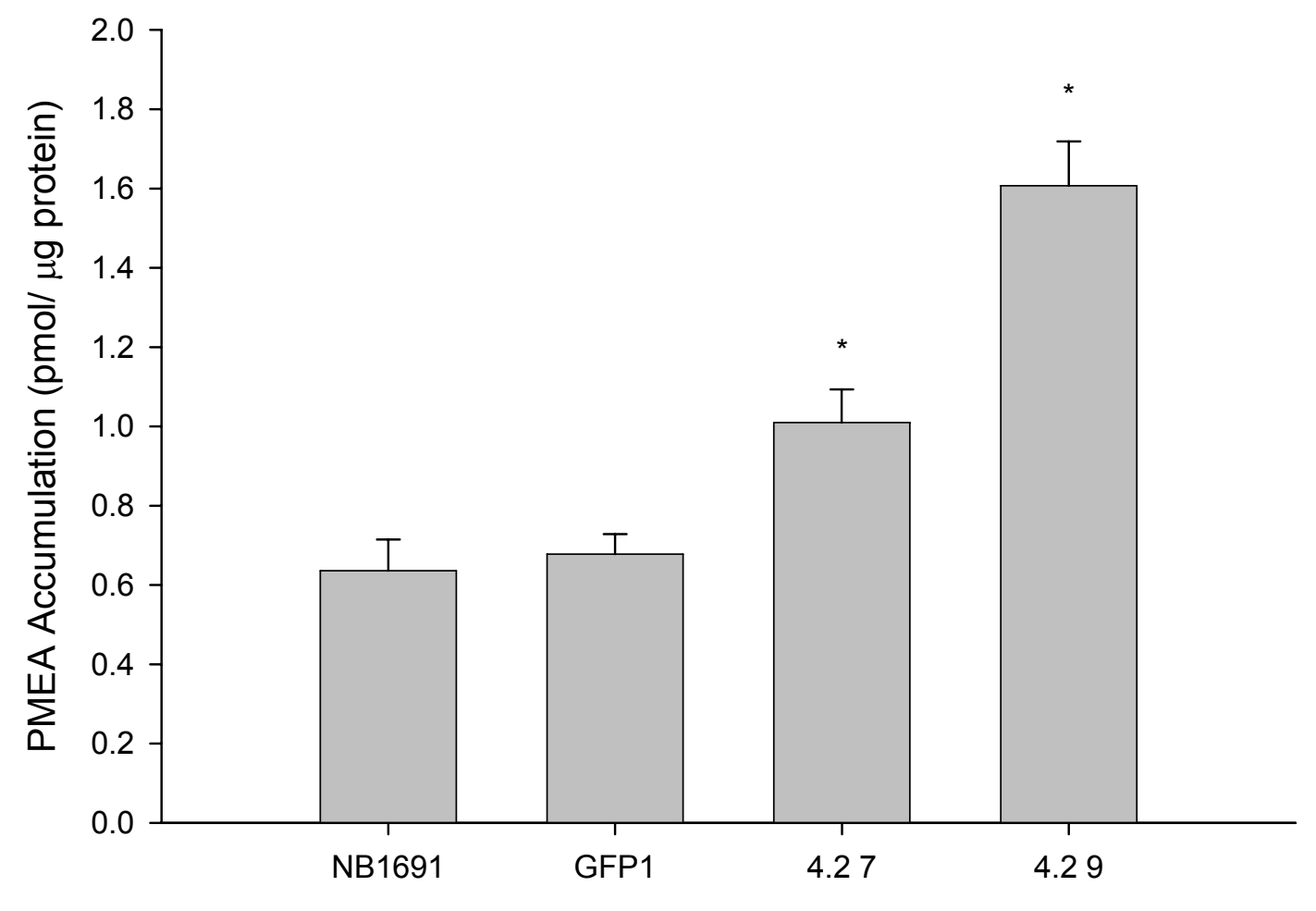

Figure 2.6 PMEA intracellular accumulation in NB1691 MRP4 shRNA clones.

Intracellular PMEA accumulation was determined after a $20 \mathrm{hr}$ incubation with BisPOM-PMEA. Reduced MRP4 expression in clones 4.27 and 4.29 results in an increase in PMEA intracellular accumulation, indicating functional knockdown of MRP4. Values are presented as mean \pm standard deviation. ${ }^{*} P \leq 0.001$ (Dunnett's test). 


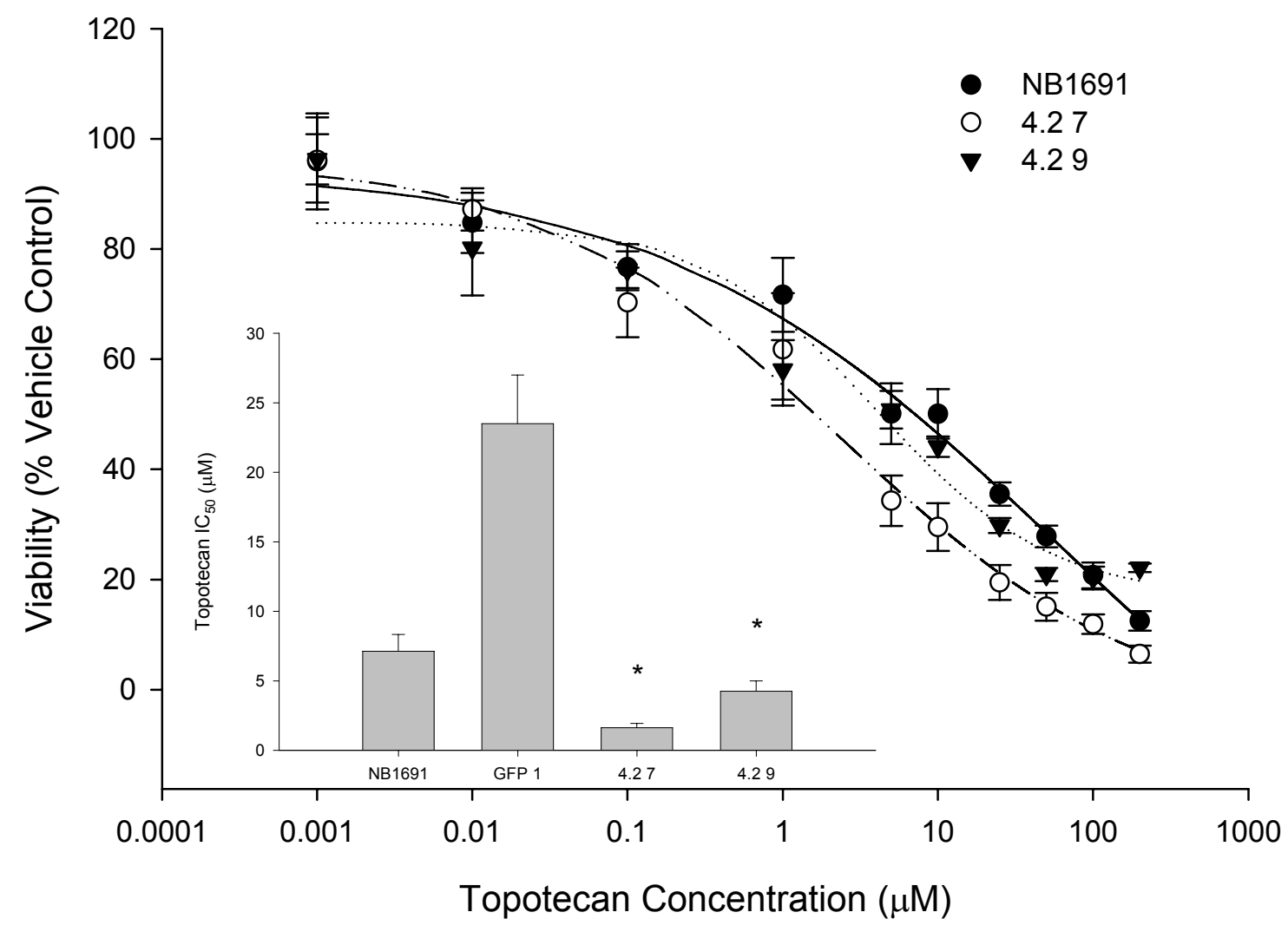

Figure 2.7 Topotecan sensitivity in NB1691 MRP4 shRNA clones.

Viability curve derived from MTT assay of NB1691 and clones with reduced MRP4 expression demonstrates increased topotecan sensitivity in clones 4.27 and 4.2 9. The 6 $\mathrm{hr}$ topotecan $\mathrm{IC}_{50}$ is lower in NB1691 clones with reduced MRP4 expression compared to the parental cell line (inset). Viability is presented as mean \pm standard deviation with the best fit line. $\mathrm{IC}_{50}$ values are presented as mean $\mathrm{IC}_{50}$ estimate (95\% confidence interval) (B). * $P \leq 0.01$ (t-test with Bonferroni correction). 
Furthermore, knocking down MRP4 expression resulted in 2-fold increased SN-38 sensitivity in clones 4.27 and 4.29 (Figure 2.8). We also evaluated the sensitivity to the prodrug irinotecan in the clones with reduced MRP4 expression, but there was no difference between the parental control and clones 4.27 and 4.29 (data not shown).

\subsubsection{Functional MRP4 overexpression in NB1643}

After demonstrating that topotecan and $\mathrm{SN}-38$ sensitivity increased upon reduction of MRP4 expression, we evaluated the effect of MRP4 overexpression in NB1643. MRP4 protein levels were 1.4- fold higher in NB1643 MRP4 IRES GFP compared to the parental cell line, as determined by densitometry (Figure 2.9). These samples were subjected to enzymatic deglycosylation because we observed slight variation in MRP4 electrophoretic migration between NB1643 and the Saos2 control cells. We reasoned that the observed differences in MRP4 molecular weight among cell lines may be due to differential glycosylation. Protein band intensity increased upon deglycosylation with $\mathrm{PNGaseF}$, indicating that the $\mathrm{M}_{4} \mathrm{I}-10$ antibody may have more specificity for deglycosylated MRP4 than the native glycoprotein.

MRP4 expression in NB1643 resulted in reduced intracellular accumulation of PMEA, indicating the presence of functional MRP4 (Figure 2.10A). PMEA accumulation in NB1643 MSCV MRP4 IRES GFP was $20 \%$ lower than in NB1643 $(P \leq 0.01)$, proportional to the MRP4 expression level. In the Saos2 MRP4 control cell line, PMEA accumulation was 2-fold lower than the vector control (Figure 2.10B). Functional expression of MRP4 in NB1643 resulted in an increase in camptothecin analog resistance. The $6 \mathrm{hr}$ topotecan $\mathrm{IC}_{50}$ was 7 -fold higher in NB1643 MRP4 compared to 


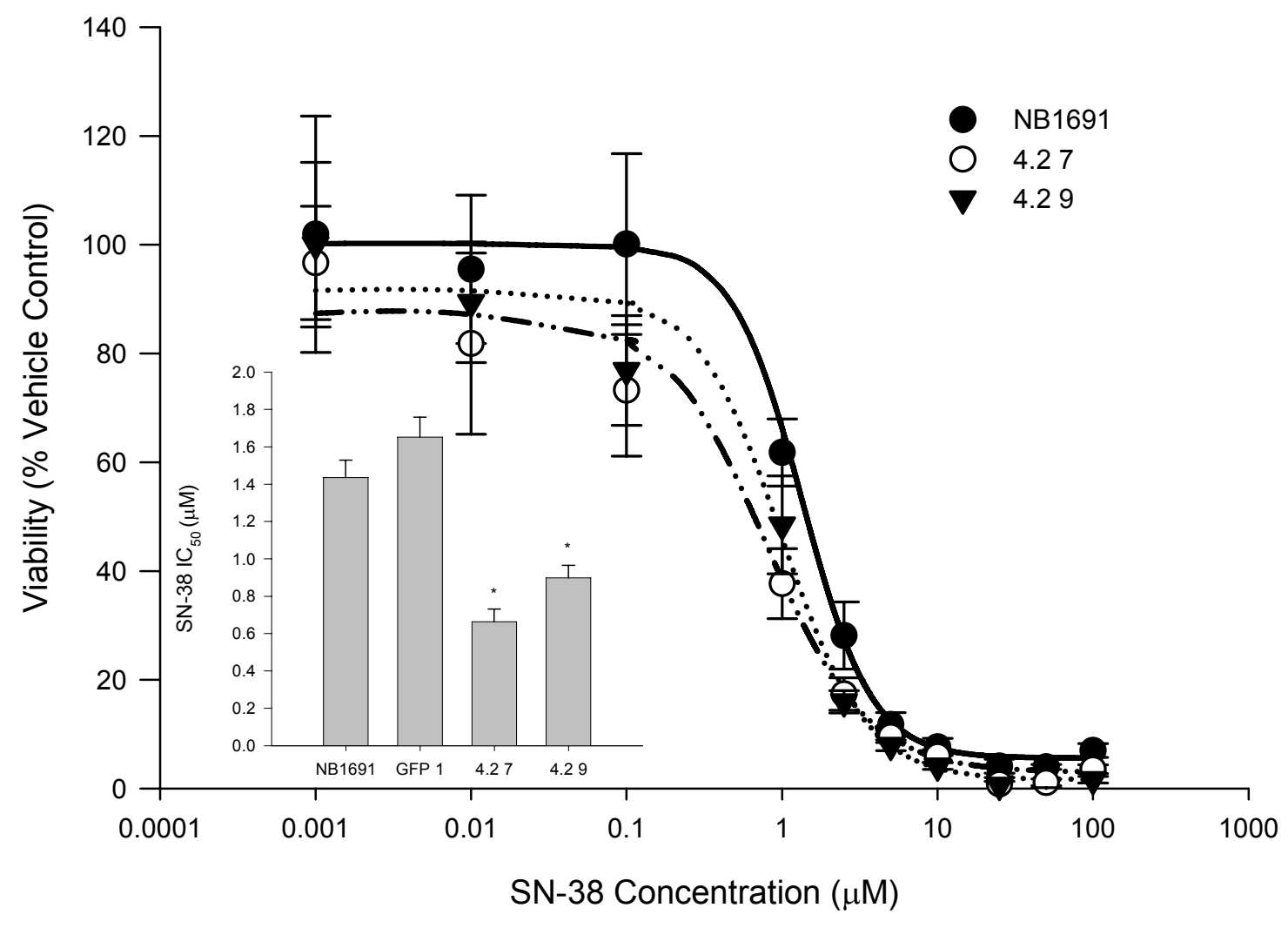

Figure 2.8 SN38 sensitivity in NB1691 MRP4 shRNA clones.

Viability curve derived from MTT assay of NB1691 and clones with reduced MRP4 expression demonstrates increased SN-38 sensitivity in clones 4.27 and 4.2 9. The $6 \mathrm{hr}$ SN-38 IC50 is lower in NB1691 clones with reduced MRP4 expression compared to the parental cell line (inset). Viability is presented as mean \pm standard deviation with the best fit line. $\mathrm{IC}_{50}$ values are presented as mean $\mathrm{IC}_{50}$ estimate ( $95 \%$ confidence interval). ${ }^{*} P \leq$ 0.01 (t-test with Bonferroni correction). 


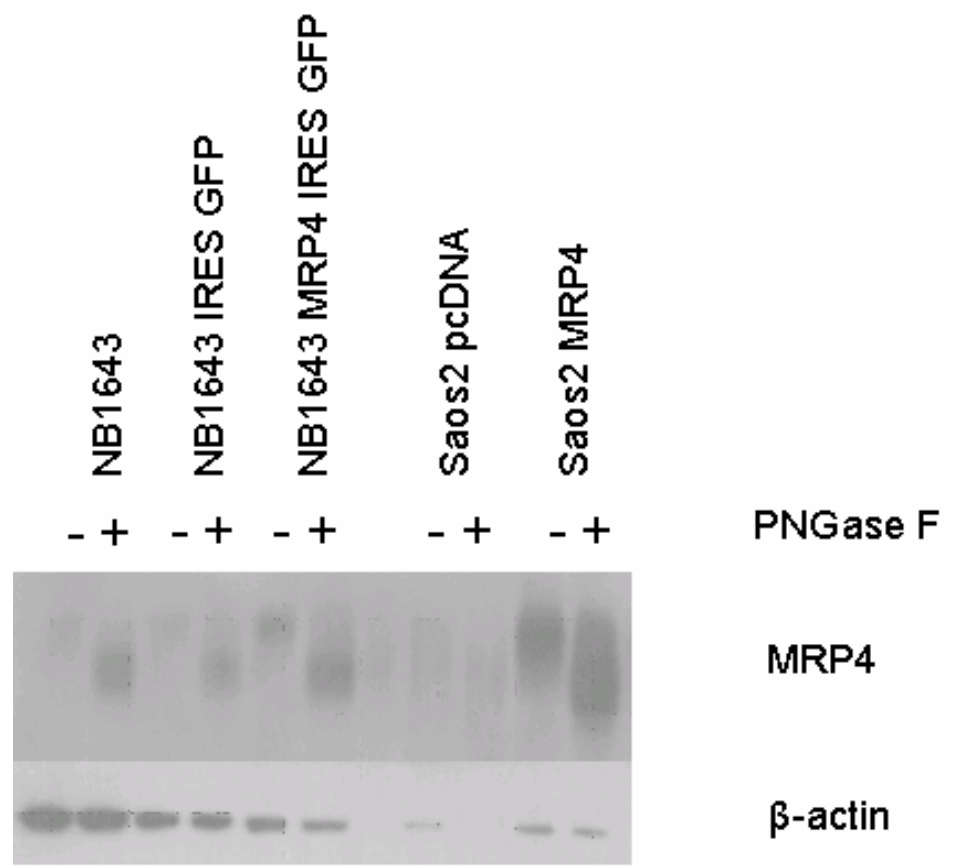

Figure 2.9 Immunoblot analysis of MRP4 expression in NB1643 cells transduced with MSCV MRP4 IRES GFP.

The blot was probed with the monoclonal $\mathrm{M}_{4} \mathrm{I}-10$ antibody. MRP4 is overexpressed in NB1643 cells transduced with MSCV MRP4 IRES GFP. PMEA accumulation is reduced in cells overexpressing MRP4. Sensitivity to topotecan and SN-38 decreases in NB1643 cells overexpressing MRP4. 
A

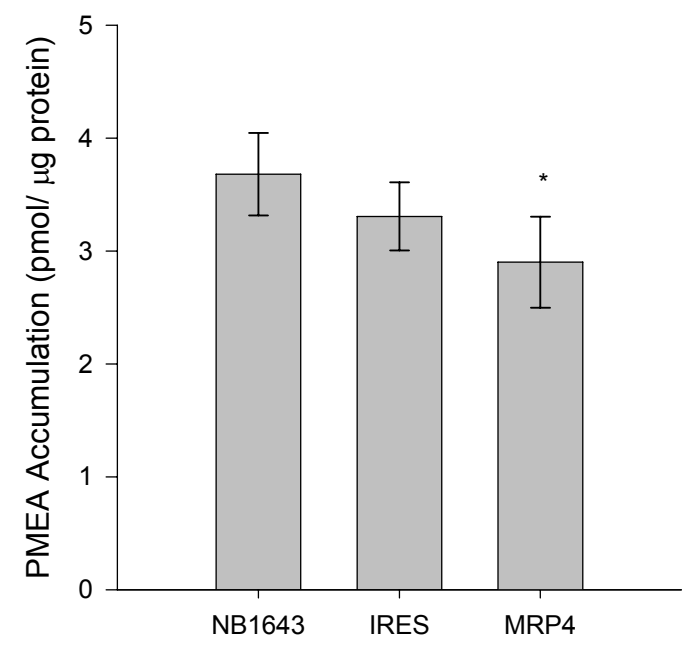

B

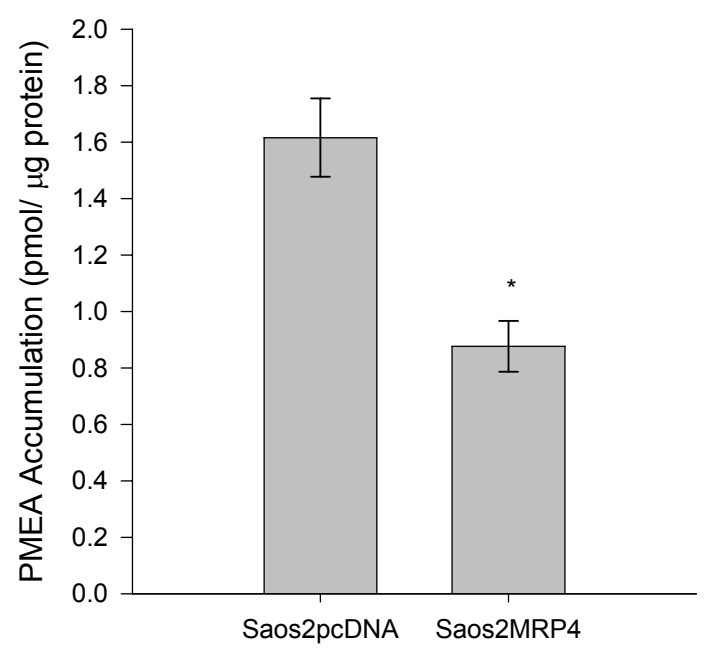

Figure 2.10 Intracellular PMEA accumulation in NB1643 cells overexpressing MRP4.

MRP4 overexpression in NB1643 results in 20\% decrease in intracellular PMEA accumulation, indicating the presence of a functional efflux transporter (A). PMEA accumulation in the control Saos2 cell line was reduced 2-fold in the MRP4 overexpressing cell line Saos2 MRP4 (B). Values are presented as mean \pm standard deviation.* $P \leq 0.005$ (Dunnett's multiple comparison test). 
NB1643 (Figure 2.11). The $6 \mathrm{hr}$ SN-38 IC $_{50}$ was 1.5-fold higher in NB1643 MRP4 compared to NB1643 (Figure 2.12).

\subsubsection{Functional Pgp knockdown with stably expressed shRNA}

Based upon the preliminary screen of six neuroblastoma cell lines in which Pgp was expressed in the most resistant cell line to topotecan, we also evaluated the effect of reducing Pgp expression in NB1691. The shRNA construct targeting MDR1 mRNA for degradation resulted in a significant decrease in MDR1 expression in clones 1, 2, and 3 (Figure 2.13).

Intracellular mitoxantrone accumulation was used to assess functional Pgp knockdown in the NB1691 MDR1 shRNA clones. Rhodamine 123 could not be used as a probe for Pgp function because rhodamine 123 and GFP fluoresce in the same channel. In the Pgp overexpressing cell line LLCPK1 MDR1, mitoxantrone accumulation was significantly reduced compared to LLCPK1, consistent with mitoxantrone as a Pgp substrate. Mitoxantrone accumulation was significantly higher (2-fold) in NB1691 MDR1 shRNA clones 1, 2, and 3 than in the parental NB1691, consistent with reduced Pgp function (Figure 2.14). Furthermore, topotecan accumulated intracellularly at higher levels in MDR1 shRNA clones 1 and 3 (Figure 2.15A). Further confirming topotecan as a Pgp substrate, topotecan accumulation was reduced in the Pgp overexpressing LLCPK1 MDR1 in comparison to the vector control (Figure 2.15B). Finally, the reduction in Pgp function and increased topotecan accumulation resulted in increased sensitivity to topotecan. The topotecan $\mathrm{IC}_{50}$ was 2- to 5-fold lower than NB1691 in MDR1 shRNA 


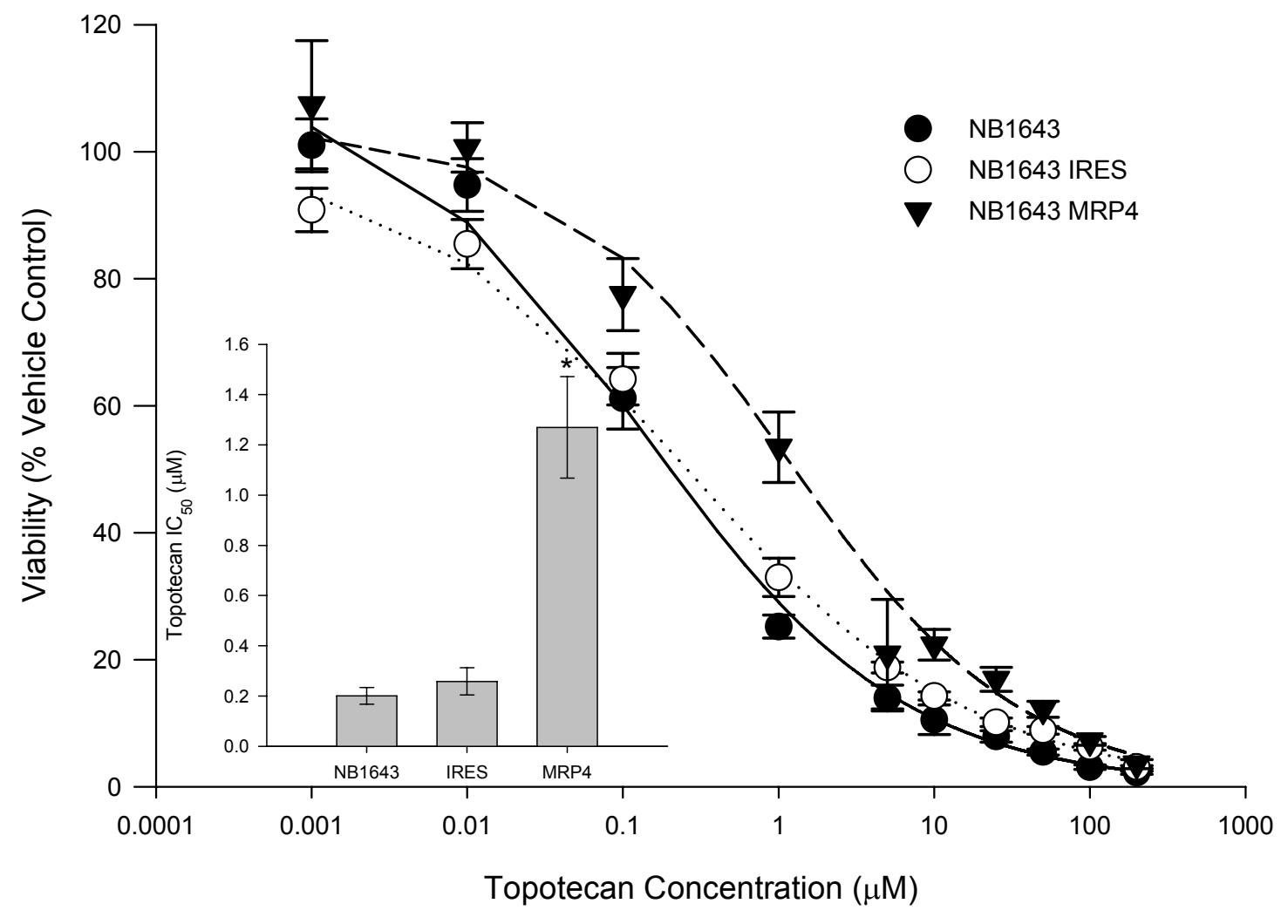

Figure 2.11 Topotecan sensitivity in NB1643 cells overexpressing MRP4.

Viability curve derived from MTT assay of NB1643, NB1643 IRES, and NB1643 MRP4 demonstrates reduced topotecan sensitivity in the cell line overexpressing MRP4. The 6 $\mathrm{hr}$ topotecan $\mathrm{IC}_{50}$ is significantly higher in NB1643 MRP4 compared to the parental cell line (inset). Viability is presented as mean \pm standard deviation with the best fit line. $\mathrm{IC}_{50}$ values are presented as mean $\mathrm{IC}_{50}$ estimate (95\% confidence interval). ${ }^{*} P \leq 0.01$ (t-test with Bonferroni correction). 


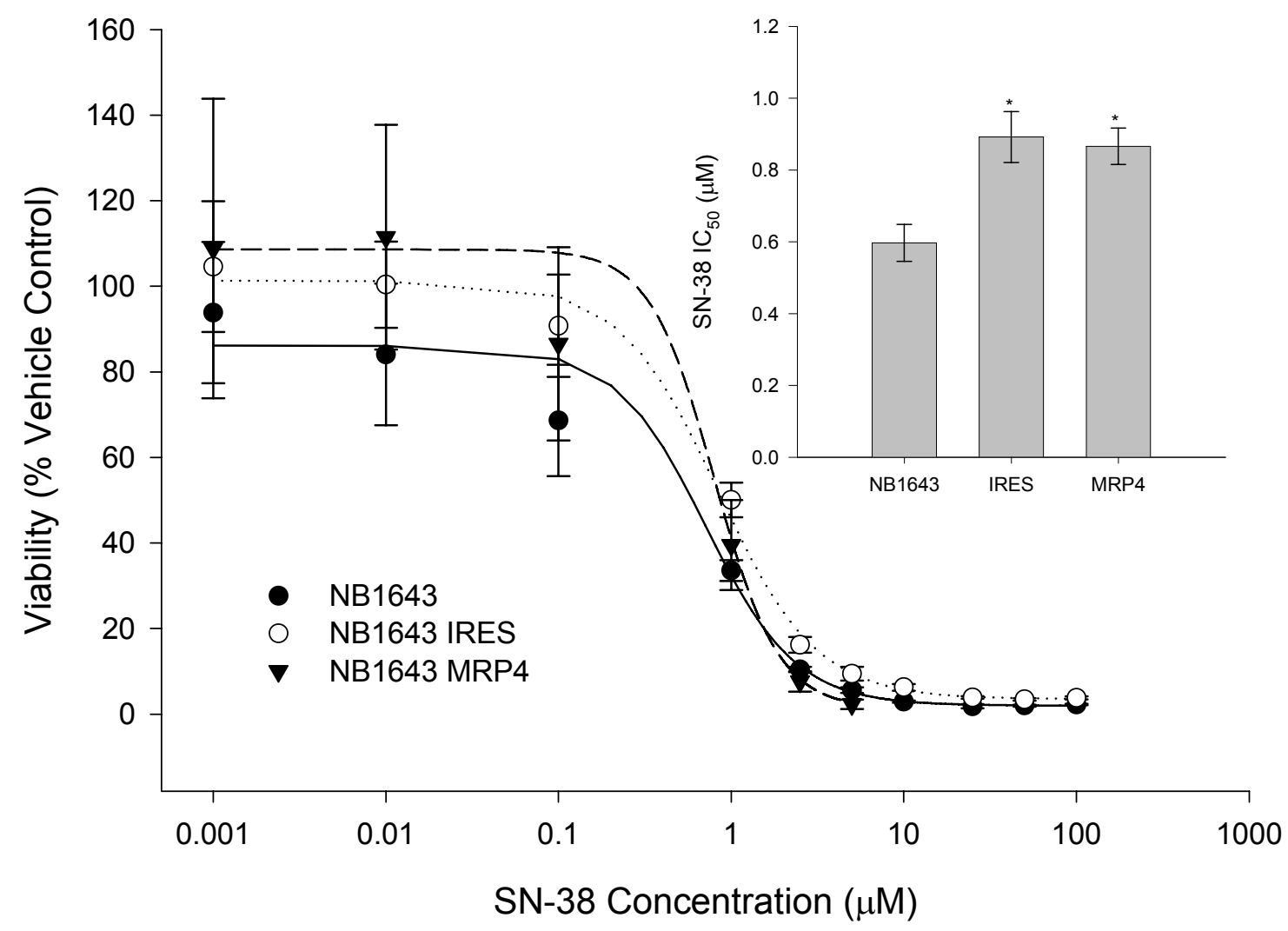

Figure 2.12 SN-38 sensitivity in NB1643 cells overexpressing MRP4.

Viability curve derived from MTT assay of NB1643, NB1643 IRES, and NB1643 MRP4 demonstrates reduced SN-38 sensitivity in the cell line overexpressing MRP4 (mean \pm standard deviation, best fit line). The $6 \mathrm{hr} \mathrm{SN}-38 \mathrm{IC}_{50}$ is significantly higher in NB1643 MRP4 compared to the parental cell line. Values are presented as mean $\mathrm{IC}_{50}$ estimate ( $95 \%$ confidence interval) (B). $* P \leq 0.01$ (t-test with Bonferroni correction). 


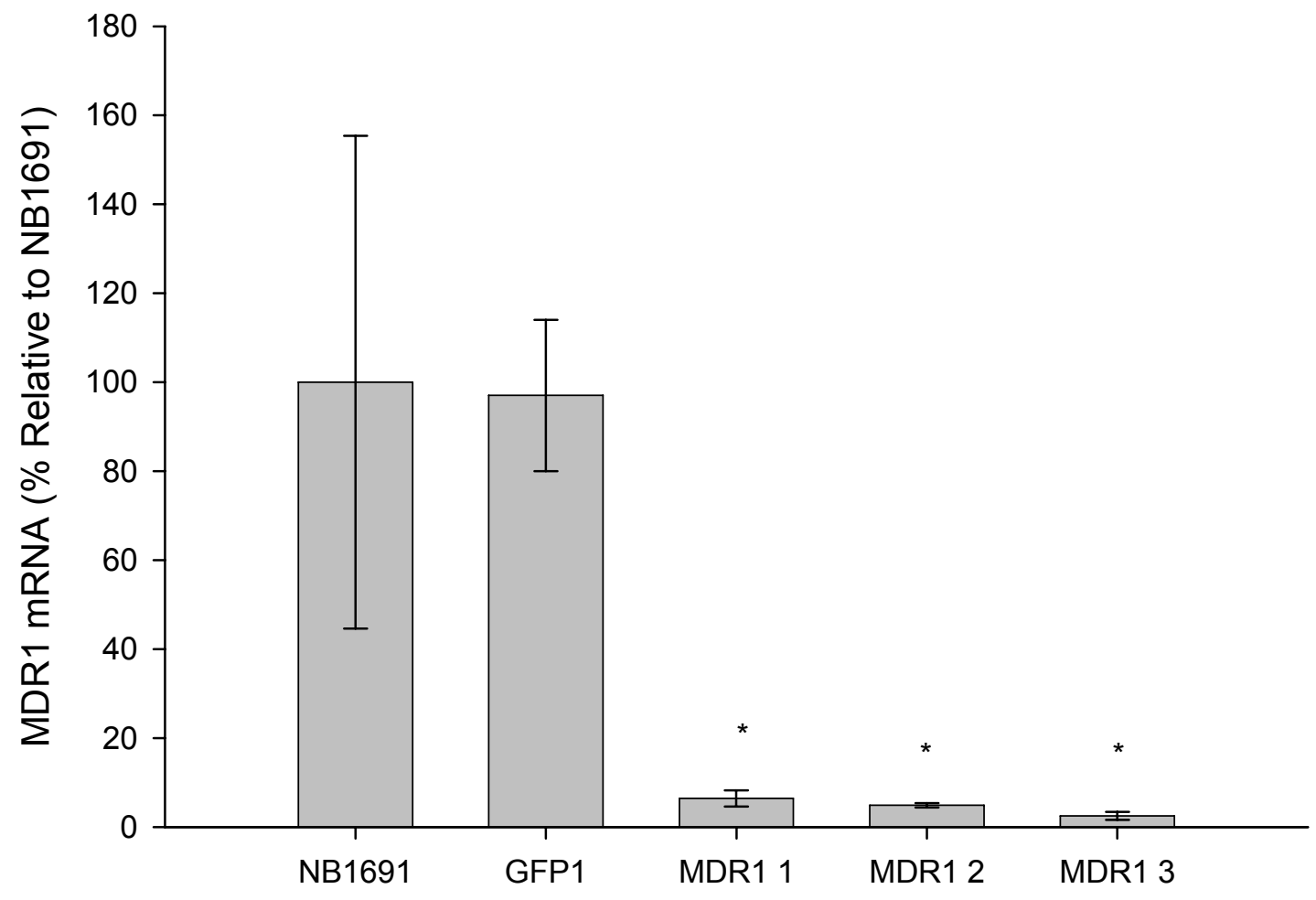

Figure 2.13 Real-time RT-PCR analysis of in NB1691 MDR1 shRNA clones.

MDR1 mRNA expression is reduced in clones 1, 2, and 3 compared to the parental cell and vector control. Values are presented as mean \pm standard deviation. ${ }^{*} \mathrm{P} \leq 0.01$ (t-test with Bonferroni correction). 
A

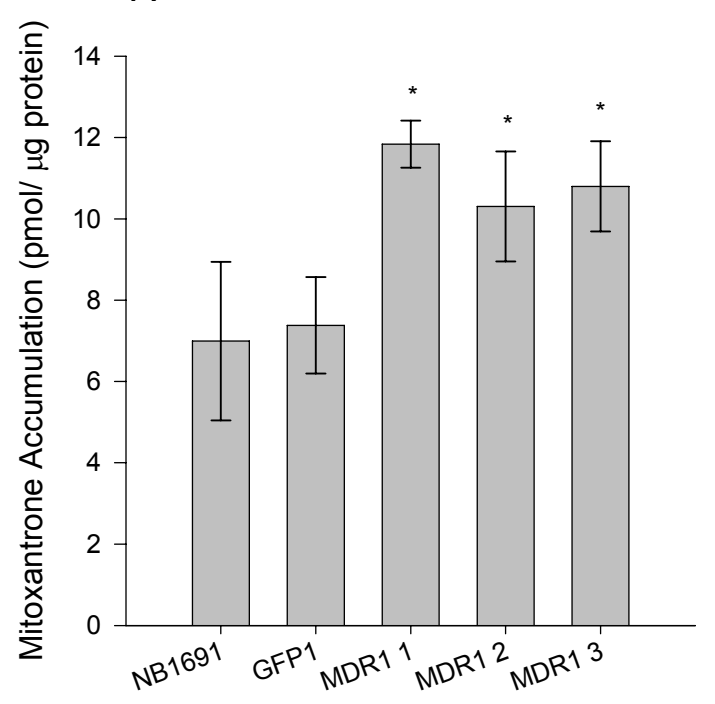

B

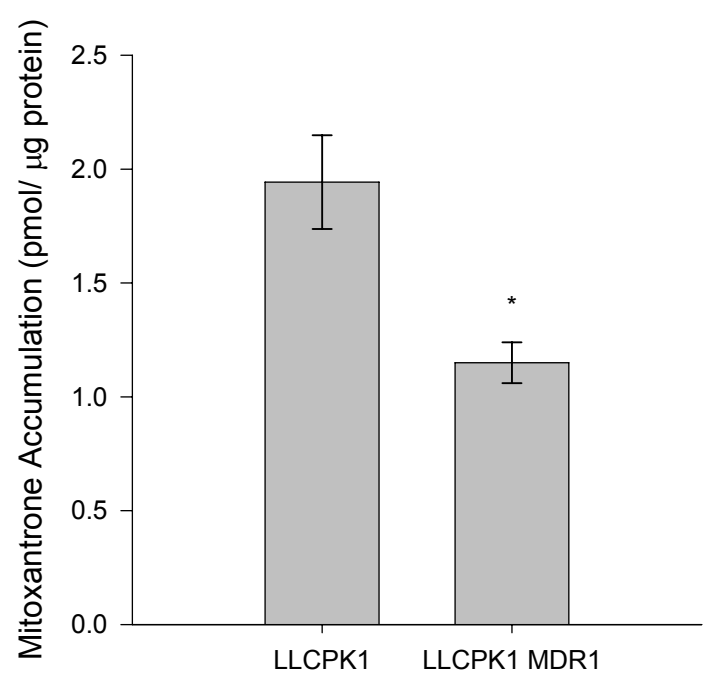

Figure 2.14 Intracellular mitoxantrone accumulation in NB1691 MDR1 shRNA clones.

Reduced Pgp expression in MDR1 shRNA clones 1, 2, and 3 results in an increase in mitoxantrone intracellular accumulation, indicating functional knockdown of Pgp (A). Mitoxantrone accumulation is reduced in the Pgp overexpressing cell line LLCPK1 MDR1 (B). Values are presented as mean \pm standard deviation. ${ }^{*} P \leq 0.001$ (Dunnett's multiple comparison test). 
A

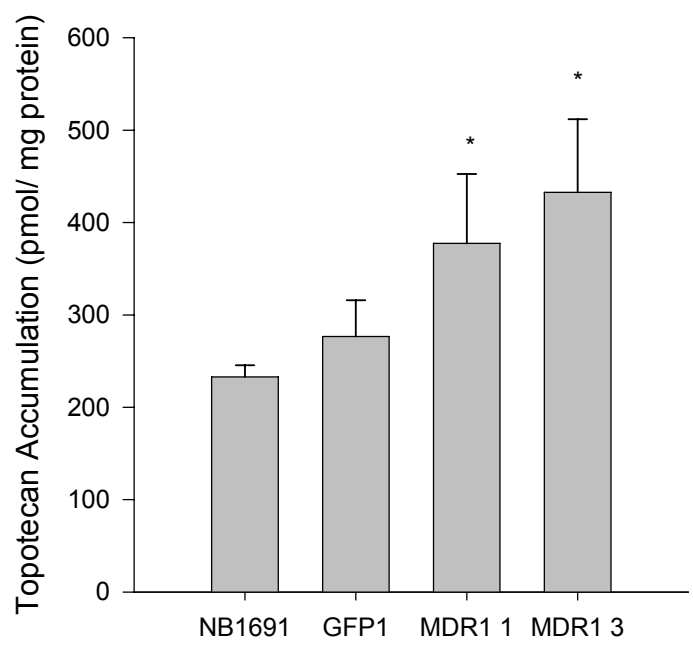

B

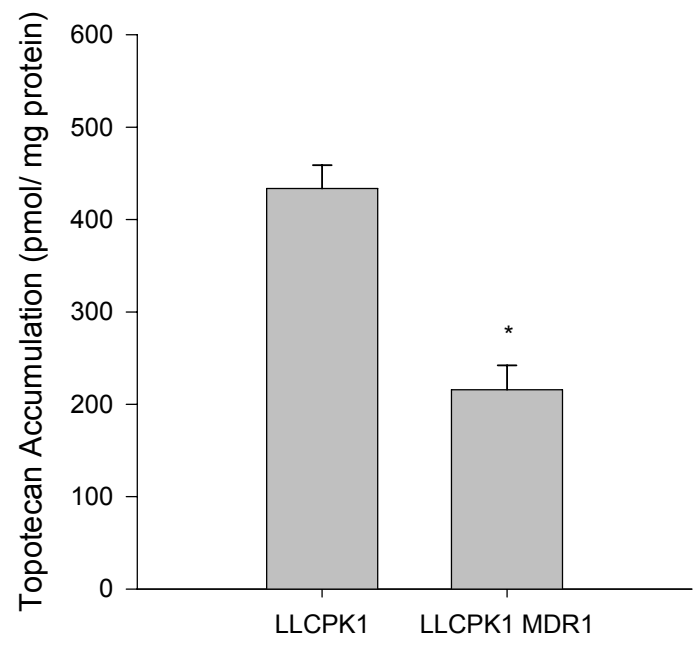

Figure 2.15 Intracellular ${ }^{14}$ C-topotecan accumulation in NB1691 MDR1 shRNA clones.

Reduced Pgp expression in MDR1 shRNA clones 1 and 3 results in an increase in topotecan intracellular accumulation (A). Topotecan accumulation is significantly reduced in the Pgp overexpressing cell line LLCPK1 MDR1 (B). Values are presented as mean \pm standard deviation. ${ }^{*} P \leq 0.001$ (Dunnett's multiple comparison test). 
clones 2 and 3 (Figure 2.16). However, reduced Pgp expression and function did not result in increased sensitivity to SN-38 (Figure 2.17). To determine if this unexpected phenotype was related to an increase in expression of other $\mathrm{ABC}$ transporters such as MRP2, we used real-time RT-PCR to evaluate mRNA expression. However, MRP2 was expressed at low levels in all of the cell lines, and no statistically significant difference was noted in expression level among the cell lines (data not shown).

\subsection{Discussion}

In this study we have shown that $\mathrm{ABC}$ transporter expression confers camptothecin analog resistance in neuroblastoma cell lines. Furthermore, reducing $\mathrm{ABC}$ transporter expression via shRNA reduces camptothecin analog resistance. The $\mathrm{ABC}$ transporters MRP4 and Pgp, which confer resistance to topotecan and SN-38 $(89,114,120,122)$, were expressed and functional in neuroblastoma cell lines relatively resistant to topotecan. In contrast, MRP4 and Pgp were not expressed in NB1643, which is relatively sensitive to topotecan. The differences in sensitivity to topotecan between NB1643 and NB1691 are not likely due to p53 status because both of these cell lines are wild-type p53 $(166,167,168)$. These in vitro results are consistent with previous in vivo xenograft studies in which NB1643 xenografts responded to a topotecan systemic exposure of $52 \mathrm{ng} / \mathrm{mL}^{*} \mathrm{hr}(61)$. In contrast, a topotecan systemic exposure of 290 $\mathrm{ng} / \mathrm{mL}^{*} \mathrm{hr}$ was required to achieve a complete response in NB1691 xenografts. The results of the current study may explain the mechanisms of camptothecin analog resistance in some neuroblastomas. 


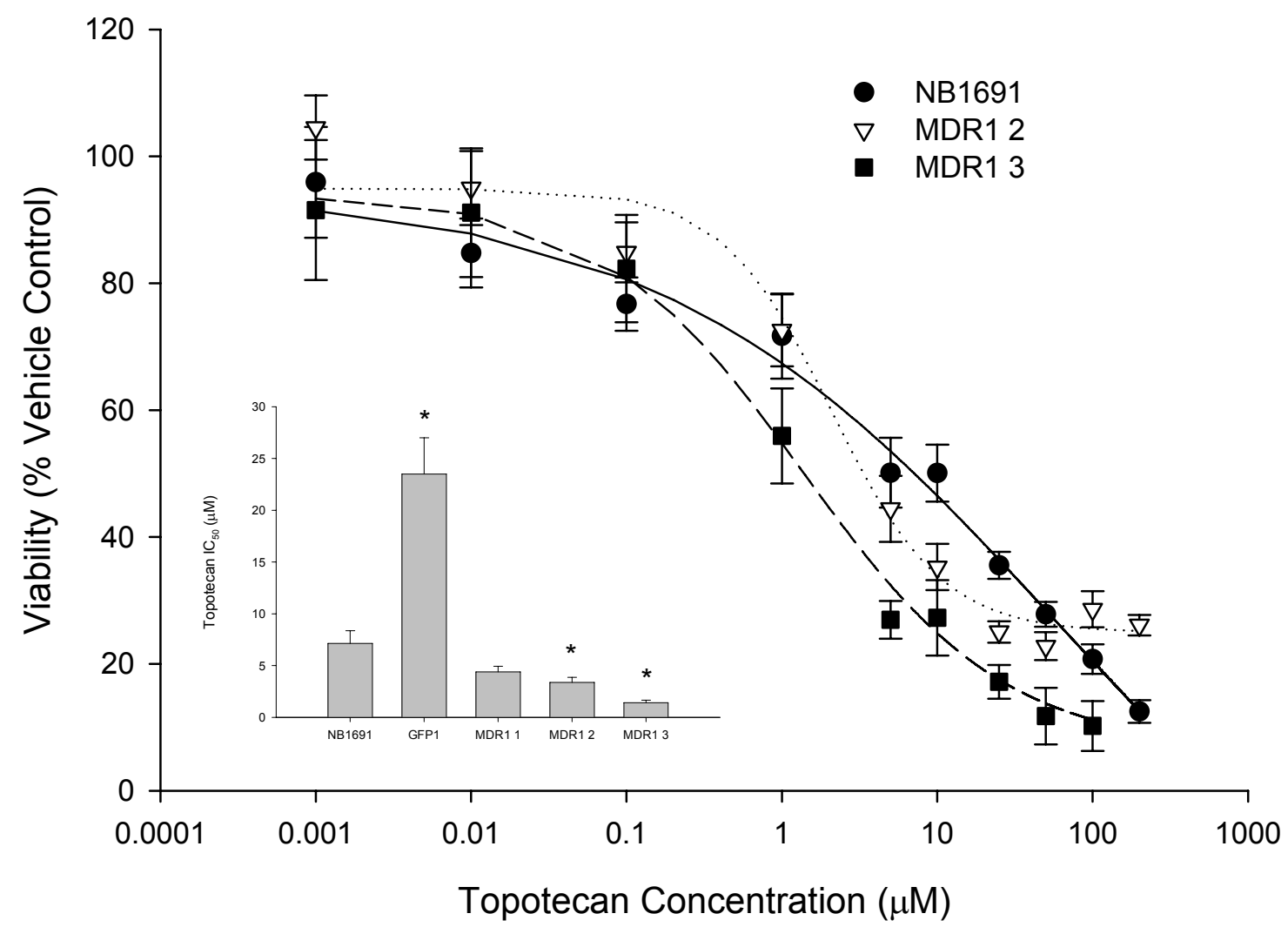

Figure 2.16 Topotecan sensitivity in NB1691 MDR1 shRNA clones

Viability curve derived from MTT assay of NB1691 and MDR1 shRNA clones 2 and 3 demonstrates increased topotecan sensitivity in clones 2 and 3 . The $6 \mathrm{hr}$ topotecan $\mathrm{IC}_{50}$ is statistically significantly lower in clones 2 and 3 expressing MDR1 shRNA compared to the parental cell line (inset). Values are presented as mean $\mathrm{IC}_{50}$ estimate $(95 \%$ confidence interval) (B). ${ }^{*} P \leq 0.01$ (t-test with Bonferroni correction). 


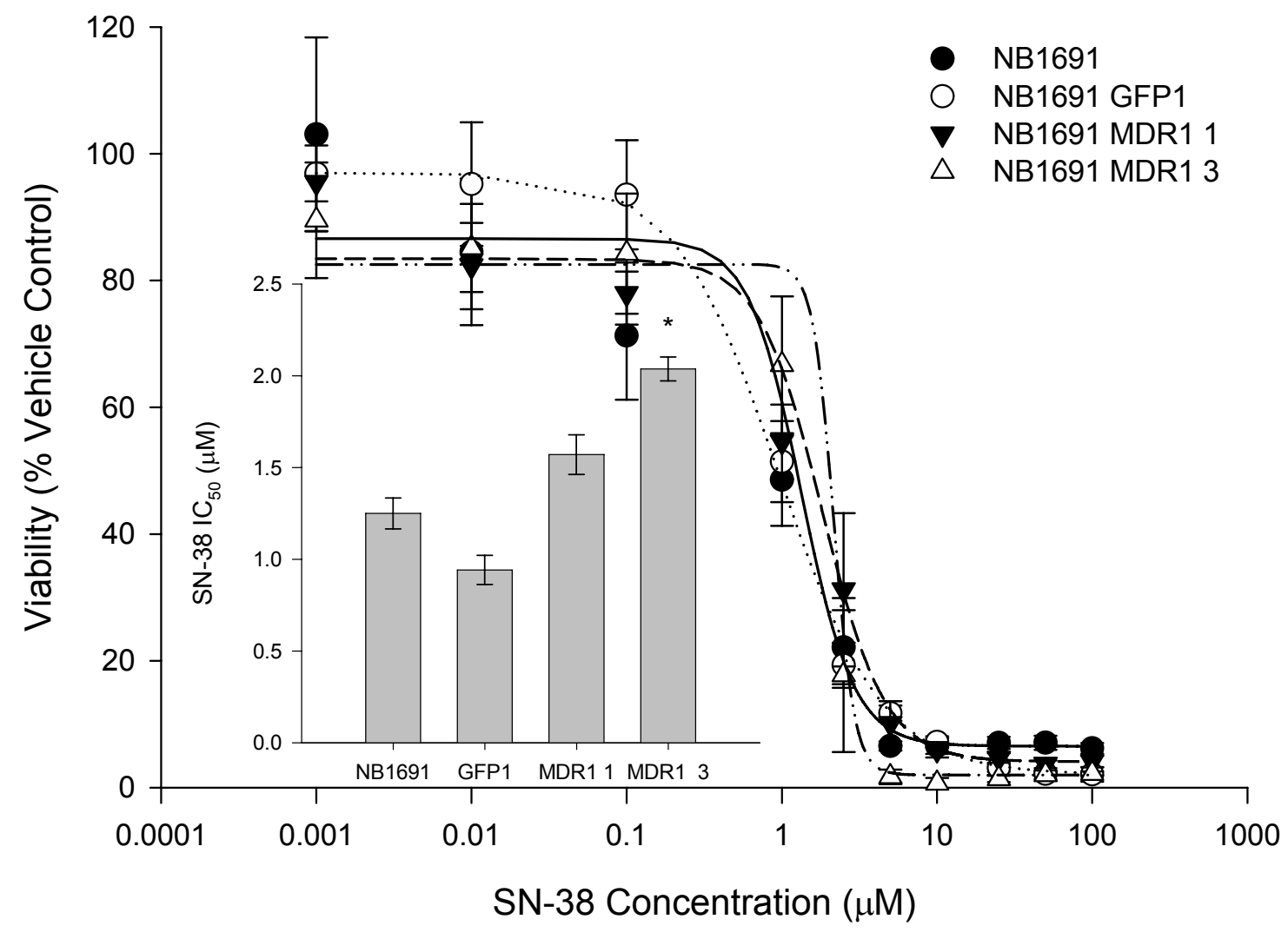

Figure 2.17 SN-38 sensitivity in NB1691 MDR1 shRNA clones.

Viability curve derived from MTT assay of NB1691 and MDR1 shRNA clones 2 and 3 demonstrates increased SN-38 resistance in clone 3. The $6 \mathrm{hr}$ topotecan $\mathrm{IC}_{50}$ is statistically significantly higher in clone 3 expressing MDR1 shRNA compared to the parental cell line (inset). Values are presented as mean $\mathrm{IC}_{50}$ estimate $(95 \%$ confidence interval) (B). ${ }^{*} P \leq 0.01$ (t-test with Bonferroni correction). 
The initial results of immunoblot analysis of $\mathrm{ABC}$ transporter expression in neuroblastoma cell lines representing a spectrum of topotecan sensitivities indicated that MRP4 and Pgp may be partly responsible for resistance to camptothecin analogs in neuroblastoma. Therefore, to determine the individual contribution of MPR4 and Pgp to camptothecin analog resistance, we used RNAi to reduce MRP4 or Pgp expression in NB1691 and evaluate the effect on drug resistance. Knocking down MRP4 expression resulted in reduced MRP4 function, i.e., increased intracellular accumulation of the MRP4 substrate PMEA and topotecan. Reduced MRP4 expression and function also resulted in increased sensitivity to both topotecan and SN-38. Moreover, exogenous MRP4 overexpression conferred topotecan and $\mathrm{SN}-38$ resistance in a neuroblastoma cell line relatively sensitive to topotecan and $\mathrm{SN}-38$.

As observed for MRP4, shRNA to Pgp reduced Pgp expression and function. In NB1691 clones with reduced Pgp expression, intracellular accumulation of mitoxantrone, a probe for Pgp function was increased. Topotecan intracellular accumulation was also increased, resulting in increased sensitivity. However, reduced Pgp functional expression did not result in an increase in $\mathrm{SN}-38$ sensitivity. We reasoned that this may be due to a compensatory increase in expression of an $\mathrm{ABC}$ transporter for which $\mathrm{SN}-38$, but not topotecan, is a substrate, such as MRP2. However, MRP2 expression did not differ among the NB1691 MDR1 shRNA clones or controls. Therefore, we postulate that the observed increase in SN-38 resistance in NB1691 MDR1 shRNA clone 3 may be due to increased intracellular inactivation of the drug.

Although neuroblastoma xenograft models and patients exhibit responses to the camptothecin analogs, there is a sensitivity continuum among both patients and 
xenografts. For example, NB1691 and NB143 were isolated from patients with stage D neuroblastoma (70). NB1643 was established from a diagnostic tumor sample. In contrast, the patient from whom NB1691 was derived had received prior therapy including cytarabine, daunomycin, 6-thioguanine, etoposide, and 5-azacytidine. This multi-agent chemotherapy may have acted as a selective pressure for a neuroblastoma clonal population that expresses $\mathrm{ABC}$ transporters. However, it is also possible that the $\mathrm{ABC}$ transporters expressed in NB1691 were present at the onset of disease and before chemotherapeutic selection of resistant clones. Without a paired tumor specimen isolated prior to therapy, it is not possible to determine whether NB1691 represents intrinsic or acquired resistance. Regardless, we have demonstrated that NB1691 is a model of ABC transporter mediated resistance to camptothecin analogs.

The findings that MRP4 and Pgp mediate resistance to the camptothecin analogs in neuroblastoma have several potential applications. A gene therapy approach using RNAi to knock down the expression of $\mathrm{ABC}$ transporters in the tumor may increase the sensitivity of neuroblastoma to topotecan or irinotecan. Reducing the expression of $\mathrm{ABC}$ transporters at the level of the tumor may also increase the sensitivity to other drugs traditionally used in neuroblastoma, such as doxorubicin, vincristine, etoposide, and cyclophosphamide. In addition to inhibiting $\mathrm{ABC}$ transporters by reducing protein expression, drugs designed to inhibit $\mathrm{ABC}$ transporters such as valspodar or biricodar may theoretically help to increase tumor levels of cytotoxic drugs. However, MDR modulators have not significantly improved outcome in clinical trials $(169,170,171)$.

In contrast to the MDR modulators biricodar or valspodar, other drugs that were not initially designed as modulators of MDR have been shown to modulate MDR as a 
"side effect." For example, the EGFR tyrosine kinase inhibitor gefitinib also inhibits BCRP and Pgp $(130,136)$. Combining gefitinib (or other similar compounds such as erlotinib) with topotecan or irinotecan can take advantage of the cytostatic effect of gefitinib in addition to its ability to increase tumor levels of cytotoxic compounds. However, gefitinib inhibits $\mathrm{ABC}$ transporters expressed in normal tissues (e.g., kidney) important in the pharmacokinetics of topotecan and irinotecan. Therefore, the interaction between camptothecin analogs and EGFR tyrosine kinase inhibitors can be three-fold: increasing camptothecin analog systemic exposure, inhibiting camptothecin analog efflux from malignant cells, and pharmacologic interaction between EGFR inhibition and topoisomerase I interaction.

Finally, the results of the current study may be useful in future trials of individualizing therapy for patients with neuroblastoma. Determining the $\mathrm{ABC}$ transporter expression profile on a diagnostic tumor sample can be used to select drugs that are not substrates for the transporters expressed in an individual patient's tumor. However, the possibility remains that no drugs currently available will be predicted to be effective in some children, especially with high-risk disease. Therefore, further development of drugs that evade the $\mathrm{ABC}$ transporters and other resistance mechanisms in children with neuroblastoma is required to improve the survival rate. 


\section{Chapter 3. Effect of MRP4 shRNA on NB1691 xenograft response to topotecan}

\subsection{Introduction}

Murine xenograft models are a useful preclinical tool for assessing response of human tumors to chemotherapeutic agents. Anti-tumor response can be measured in xenograft-bearing mice treated with various drugs or drug combinations. Furthermore, various dosages and schedules of drug administration can be compared in murine xenograft models.

A panel of six neuroblastoma xenograft models demonstrated a range of responses to topotecan (155). A complete response to 3 intravenous topotecan courses administered daily for 5 days for two weeks $([(\mathrm{dx} 5) 2] 3)$ was achieved in 4 of the 6 xenograft models at a dosage of $0.61 \mathrm{mg} / \mathrm{kg}$. In contrast to the xenografts that responded to topotecan, the relatively resistant neuroblastoma xenograft model NB1691 required a topotecan dosage as high as $2 \mathrm{mg} / \mathrm{kg}$ to achieve a complete response. But a partial response to this $[(\mathrm{d} \times 5) 2] 3$ topotecan schedule was observed in NB1691 at a dosage of 1 $\mathrm{mg} / \mathrm{kg}$.

Efflux of topotecan by $\mathrm{ABC}$ transport proteins has been implicated in resistance to topotecan. In a screen of $\mathrm{ABC}$ transport protein expression of neuroblastoma cell lines cultured in vitro, we demonstrated that the relatively resistant NB1691 expressed both MRP4 and Pgp (172), and topotecan is a substrate for both MRP4 and Pgp (114). However, the relatively sensitive NB1643 did not express MRP4 or P-glycoprotein. Therefore, we hypothesized that knocking down MRP4 or P-glycoprotein expression in 
NB1691 would increase the sensitivity of NB1691 to camptothecin analogs and yield a response profile similar to NB1643 both in vitro and in vivo. As described in Chapter 2, the in vitro studies of topotecan sensitivity in NB1691 in which shRNA was used to reduce MRP4 expression demonstrated an increase in topotecan sensitivity.

The objective of this study was to evaluate the contribution of MRP4 expression in neuroblastoma to tumor response to topotecan in vivo. Therefore, we established the NB1691 MRP4 shRNA clones as xenografts in SCID mice. We measured the xenograft response to topotecan $[(\mathrm{d} \times 5) 2] 3$ at dosages previously shown to elicit partial and complete responses in the NB1691 xenograft model.

\subsection{Materials and methods}

\subsubsection{Knockdown via stable shRNA}

MRP4 expression in NB1691 was knocked down in vitro using shRNA as described in Chapter 2. Single cell clones were selected by FACS, expanded, and characterized by Western blot and in vitro cytotoxicity assays. Cells $\left(1 \times 10^{8}\right)$ were harvested and transplanted into the flanks of CB17/Icr female scid $^{-/}$mice.

\subsubsection{Growth inhibition studies}

Mice bearing s.c. tumors received topotecan when tumors were $\sim 0.20$ to $1 \mathrm{~cm}$ in diameter (173). Tumor diameters were measured weekly using Vernier calipers. Assuming tumors to be spherical, tumor volumes were calculated according to the

formula $\left[(\pi / 6) \times d^{3}\right]$ where $d$ is the mean diameter. Tumor bearing mice were randomized into groups of ten prior to therapy. One group was treated with vehicle control, and a 
second group was treated with topotecan. All mice were housed under barrier conditions. All experiments were conducted using protocols and conditions approved by the St. Jude Children's Research Hospital Institutional Animal Care and Use Committee.

\subsubsection{Drugs and formulation}

Topotecan (GlaxoSmithKline, King of Prussia, PA) was formulated in $0.9 \%$ saline and administered i.v. (0.05 mL/10g body weight) at a dose of 1 or $2 \mathrm{mg} / \mathrm{kg}$ as a short injection $(<1$ minute) into the lateral tail vein. Mice received topotecan $[(d x 5) 2] 3$.

\subsubsection{Real-time RT-PCR}

RNA was extracted from xenograft tissue with Tri-Reagent (Molecular Research Center, Cincinnati, $\mathrm{OH}$ ), and cDNA was synthesized using a mixture of oligo-dT and random hexamer primers with the QuantiTect Reverse Transcription Kit (Qiagen, Valencia, CA). Real-time PCR analysis was conducted using gene specific primers as described in Chapter 2. Thermal cycling conditions were as follows: $15 \mathrm{~min}$ at $95^{\circ} \mathrm{C}$ followed by 40 cycles of $95^{\circ} \mathrm{C}$ for $30 \mathrm{~s}, 60^{\circ} \mathrm{C}$ for $30 \mathrm{~s}, 72^{\circ} \mathrm{C}$ for $30 \mathrm{~s}$, and finally a denaturation stage. The mRNA expression level was determined by the comparative $\mathrm{C}_{t}$ method. Student's t-test (two-tailed) with Bonferroni correction was used to determine differences in mRNA expression between NB1691 and MRP4 shRNA clones.

\subsubsection{Immunohistochemistry}

Neuroblastoma xenograft tissues were fixed in formalin and embedded in paraffin. Sections $(4 \mu \mathrm{m})$ were placed on positively charged slides. Slides were heated $\left(60^{\circ} \mathrm{C}\right)$ for 30 minutes and cleared of paraffin in CitriSolv (Fisher Scientific, Hampton, 
$\mathrm{NH}$ ) and alcohol gradient solution panel prior to high temperature antigen recovery in citrate buffer $\mathrm{pH}$ 6.0. Slides were blocked with 3\% hydrogen peroxide in methanol and avidin/ biotin blocking reagent (Vector Labs, Burlingame CA) prior to incubation with the primary antibody. Irrelevant isotype fractions were used as negative controls. Biotinylated secondary antibodies were used (Vector Labs), and the tertiary reagent was streptavidin-horseradish peroxidase (Dako, Carpinteria, CA). Color development was achieved with diaminobenzidine (DAB, Dako), and the slides were counterstained with hematoxylin (Dako).

\subsection{Results}

As described in Chapter 2, the retroviral-vector mediated MRP4 shRNA reduced MRP4 mRNA and protein expression in NB1691 clones 4.27 and 9 in vitro. When these clones were transplanted into SCID mice as xenografts, all 4 cell lines (NB1691 parental, GFP1 vector control, and clones 4.27 and 9) grew and developed palpable tumors. However, on the second passage in vivo, clone 4.27 did not develop palpable tumors.

To determine the effect of reducing MRP4 expression in NB1691 on xenograft response to topotecan, mice were treated at a topotecan dosage and schedule that had previously elicited a partial response in NB1691 (1 mg/kg [(dx5)2]3). We hypothesized that reduced MRP4 expression would be associated with a more pronounced antitumor response in comparison to the parental cell line. Unexpectedly, none of the tumors responded to topotecan $1 \mathrm{mg} / \mathrm{kg}$ (Figure 3.1). Therefore, we treated the xenografts on the same schedule at a higher topotecan dosage $(2 \mathrm{mg} / \mathrm{kg})$. Again, unexpectedly none of the xenografts responded to the higher topotecan dosage (Figure 3.2). NB1691GFP 4.27 was 

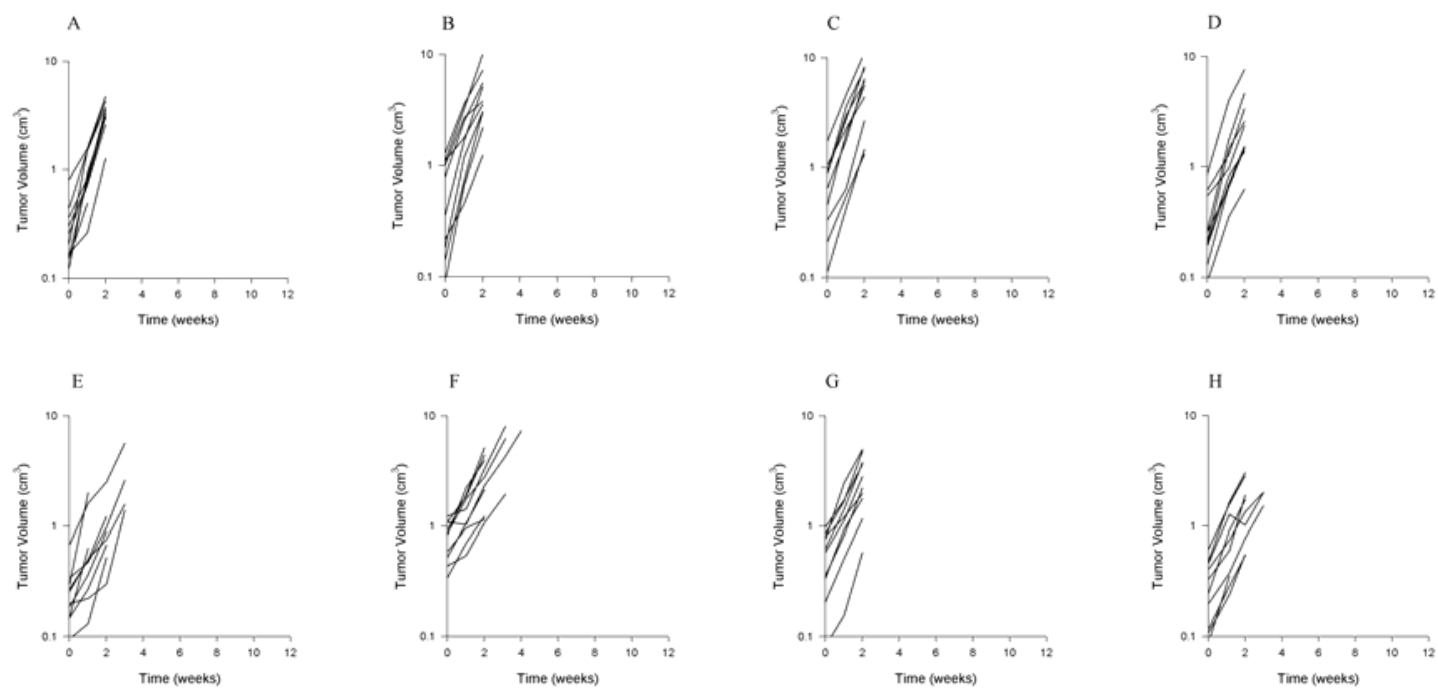

Figure 3.1 NB1691 MRP4 shRNA xenograft response to i.v. topotecan $1 \mathrm{mg} / \mathrm{kg}$ [(dx5)2]3.

Tumor volume was measured weekly until tumor volume quadrupled. Mice were treated with vehicle control (A-D) or topotecan daily for 5 days for 2 weeks every 21 days for up to 3 courses (E-H). NB1691 (A, E); NB1691 GFP1 (B, F); NB1691 GFP 4.27 (C, G); NB1691 GFP 4.29 (D, H). Each curve represents the growth of an individual tumor. 
A

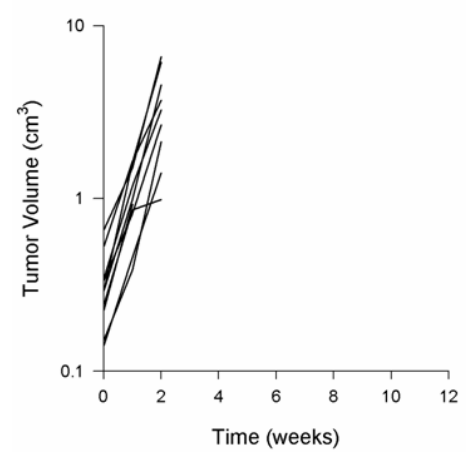

D

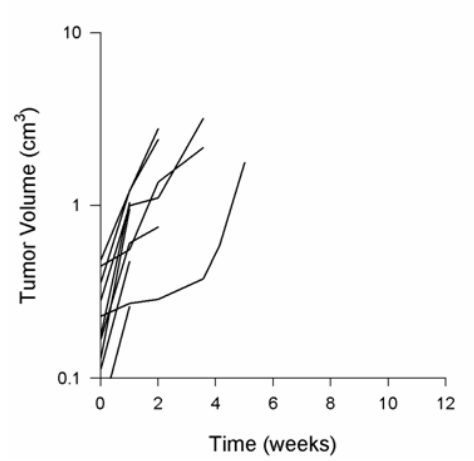

B

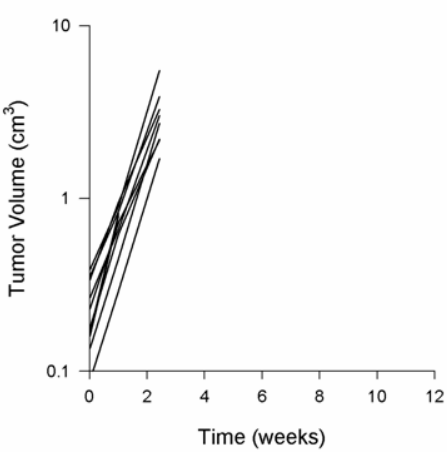

$\mathrm{E}$

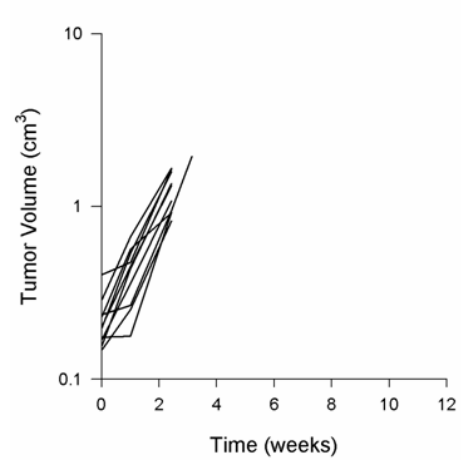

$\mathrm{C}$

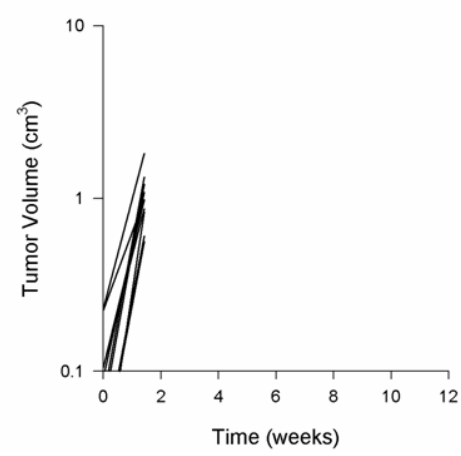

F

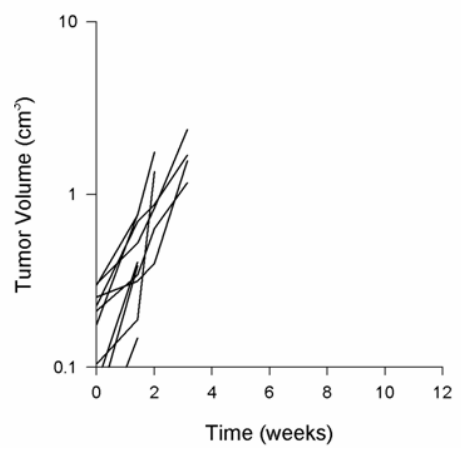

Figure 3.2 NB1691 MRP4 shRNA xenograft response to i.v. topotecan $2 \mathrm{mg} / \mathrm{kg}$ [(dx5)2]3.

Tumor volume was measured weekly until tumor volume quadrupled. Mice were treated with vehicle control (A-C) or topotecan daily for 5 days for 2 weeks every 21 days for up to 3 courses (D-F). NB1691 (A, D); NB1691 GFP1 (B, E); NB1691 GFP 4.29 (C, F). Each curve represents the growth of an individual tumor. 
not included at the $2 \mathrm{mg} / \mathrm{kg}$ dosage because the xenografts did not grow after the second passage in vivo.

To determine if ineffective MRP4 knockdown was related to failure to respond in the NB1691 xenograft clones, we evaluated the MRP4 mRNA and protein expression in the xenografts. In clone 4.27 , real-time RT-PCR analysis demonstrated that MRP4 mRNA expression was approximately half the mRNA level in the parental xenograft (Figure 3.3). Whereas, in vitro, the MRP4 mRNA expression level in clone 4.27 was only $10 \%$ of the parental cell line (Figure 2.5). However, in clone 4.29 MRP4 mRNA expression was not significantly reduced compared to NB1691 (Figure 3.3), indicating that the MRP4 shRNA did not persist in this clone. Microscopic evaluation of xenograft sections revealed green fluorescence, indicating that the GFP cassette had not been silenced. Immunohistochemical staining of MRP4 in the NB1691 xenografts demonstrated heterogeneous MRP4 expression in all four xenografts (Figure 3.4). Therefore, effective MRP4 knockdown via shRNA did not persist in this in vivo xenograft model.

Upregulation of other ABC transporters for which topotecan is a substrate may be responsible for the lack of response in the MRP4 shRNA xenografts. Therefore we also evaluated MDR1 and BCRP mRNA expression. MDR1 mRNA expression was only significantly elevated in the GFP1 vector only xenograft (Figure 3.5). However, the observed level of MDR1 expression in all four xenograft models may be sufficient to confer in vivo resistance to topotecan. Real-time RT-PCR analysis demonstrated that BCRP mRNA was expressed at low levels $\left(C_{t}\right.$ of approximately 30$)$ in all four xenograft models, and no significant difference was observed in expression level among them (data 


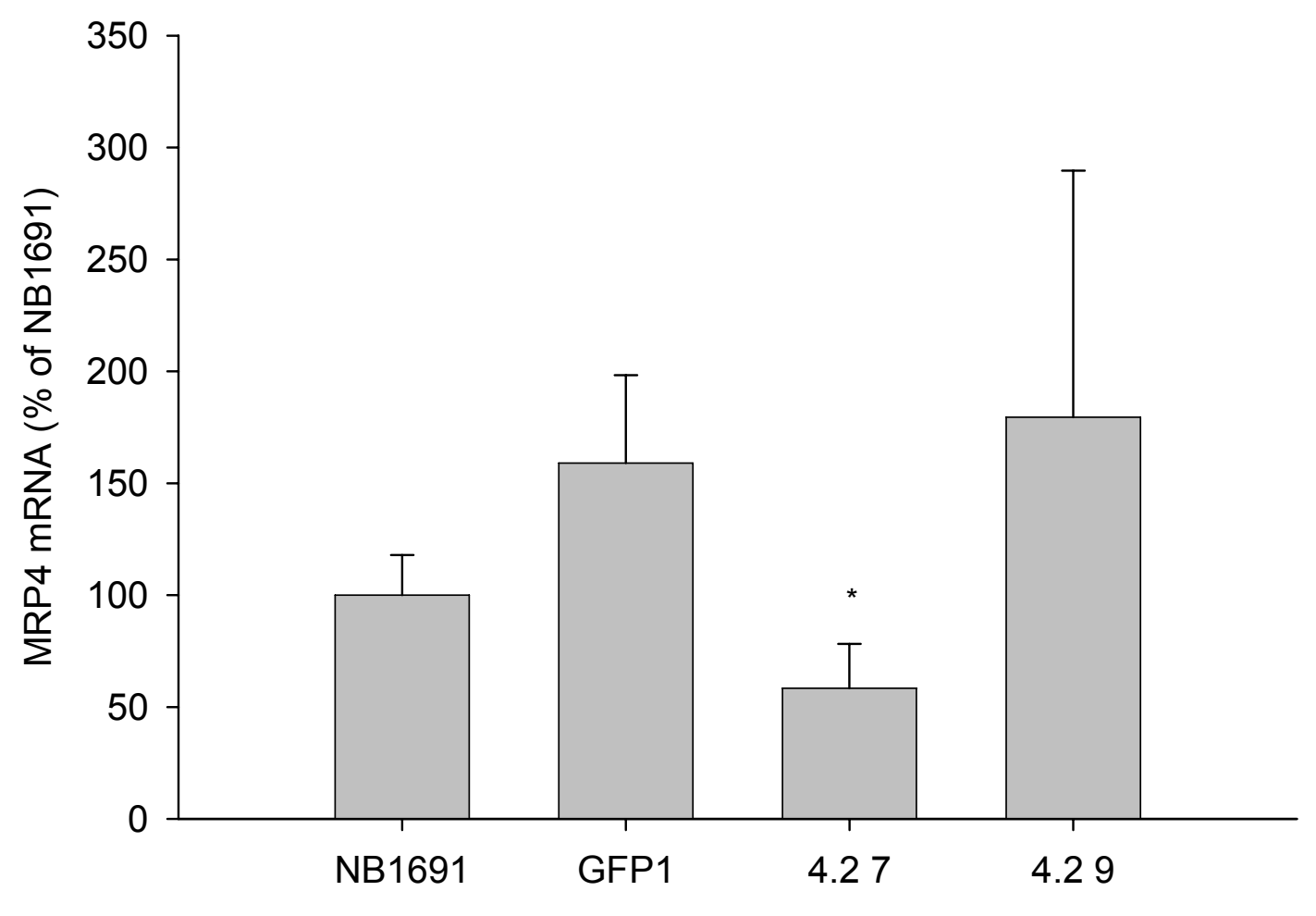

Figure 3.3 MRP4 mRNA expression in NB1691 MRP4 shRNA xenografts.

MRP4 mRNA expression is significantly reduced in NB1691 4.27 but not $4.29 .{ }^{*} P<0.01$ (t-test with Bonferroni correction). 


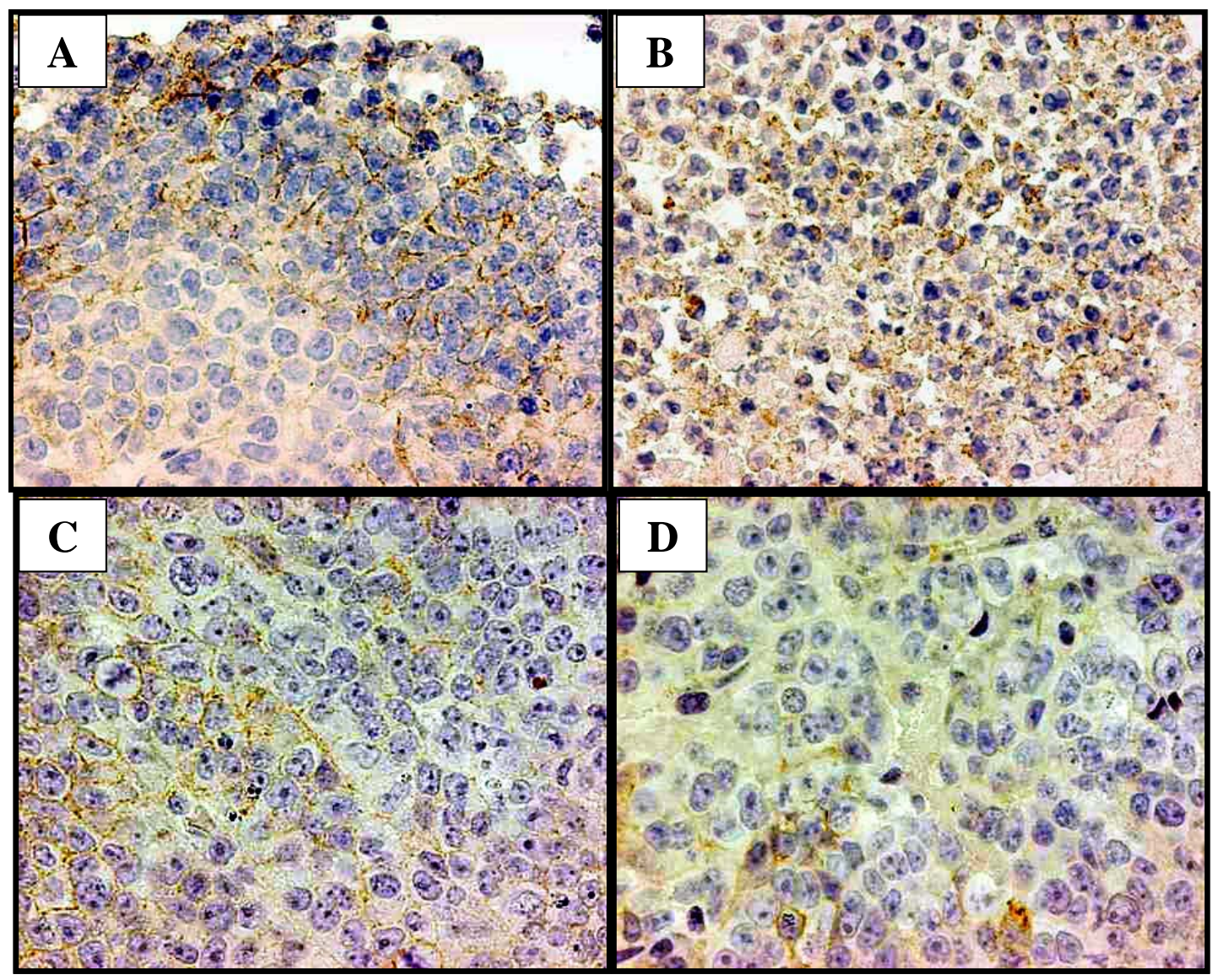

Figure $3.4 \quad$ MRP4 is expressed in NB1691 MRP4 shRNA xenografts.

Immunohistochemical analysis of NB1691 MRP4 shRNA xenografts showing heterogeneous membrane staining of MRP4 (brown stain). NB1691 (A); NB1691 GFP1 (B); NB1691 4.27 (C); NB1691 4.2 9(D). MRP4 shRNA did not effectively reduce the MRP4 expression in NB1691 xenografts. 


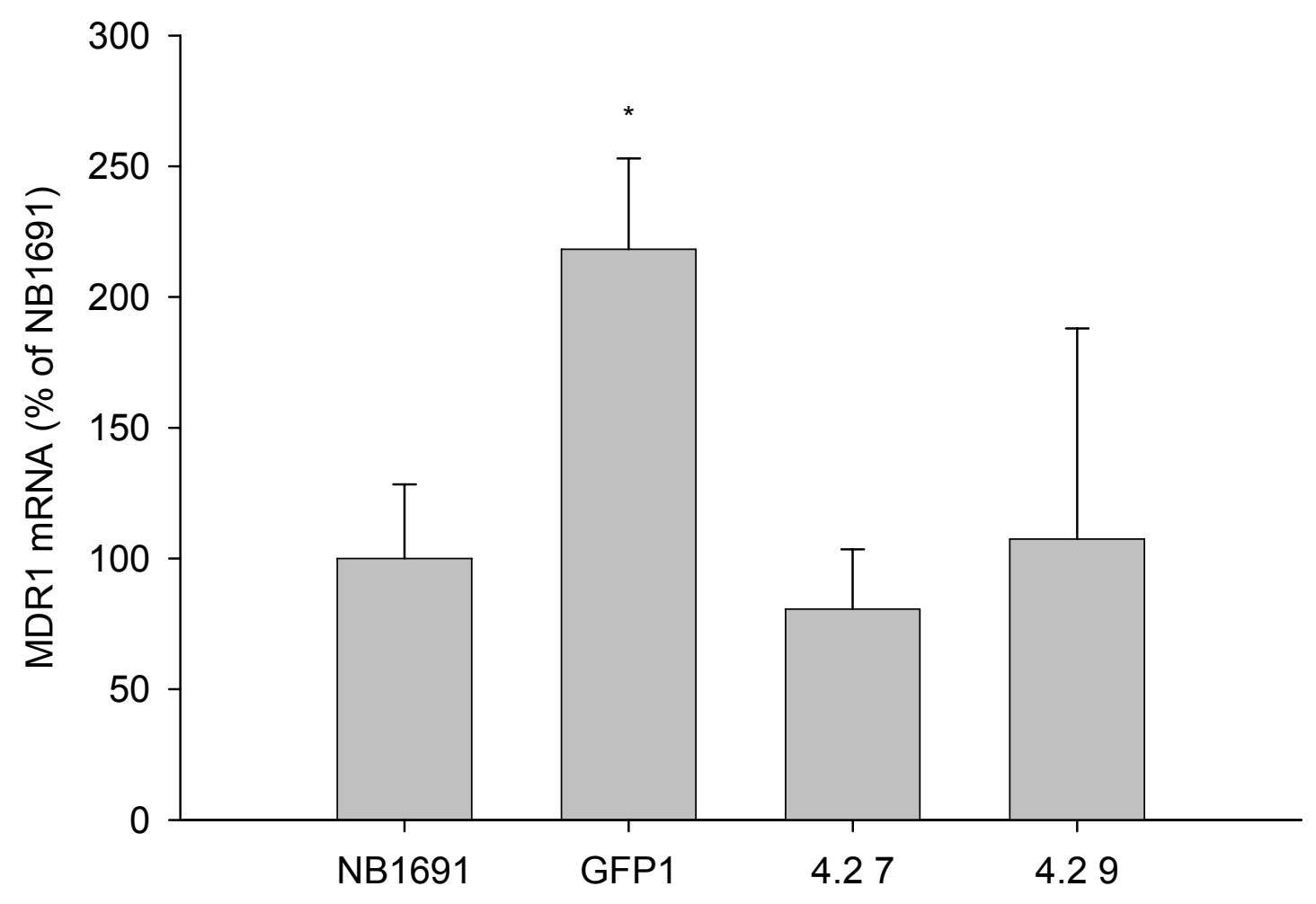

Figure 3.5 MDR1 mRNA expression in NB1691 MRP4 shRNA xenografts.

MDR1 mRNA expression is significantly increased in NB1691 GFP1. ${ }^{*} P<0.01$ (t-test with Bonferroni correction). 
not shown). BCRP was not expressed in the four xenograft models as determined by immunohistochemical analysis. Therefore, in vivo expression of BCRP does not account for the failure of these xenografts to respond to topotecan.

\subsection{Discussion}

In order to determine the contribution of MRP4 expression in vivo to response to topotecan, we established the NB1691 clones transduced with MRP4 shRNA as xenografts. SCID mice were treated with a protracted topotecan schedule that had previously elicited a partial response in CBA/CaJ mice bearing NB1691 xenografts (61).Unexpectedly, none of the 4 xenograft models responded to 1 or $2 \mathrm{mg} / \mathrm{kg}$ topotecan [(dx5)2]3. To determine if MRP4 silencing via shRNA persisted in xenografts, we analyzed MRP4 expression by real-time RT-PCR and immunohistochemistry. MRP4 mRNA expression was higher in vivo than in vitro. In addition, results of immunohistochemical analysis demonstrated that MRP4 was heterogeneously expressed in all four xenografts. Therefore, effective MRP4 shRNA did not persist in vivo. However, expression of MRP4 in these four xenograft models and failure to respond to topotecan are consistent with the hypothesis that MRP4 expression confers resistance to topotecan.

The lack of response to topotecan of the parental NB1691 xenograft may be attributable to variations in tumor growth or response in different strains of mice. The partial response of NB1691 to $1 \mathrm{mg} / \mathrm{kg}$ topotecan was observed in CBA/CaJ mice (61). The current study was conducted in SCID mice. Thompson and colleagues demonstrated that NB1691 xenografts in SCID mice were less sensitive to topotecan than in the CBA/CaJ host (174). 
Topotecan pharmacokinetics are similar in SCID and CBA/CaJ mice (Dr. Clinton Stewart, unpublished data), so the difference between strains is not attributable to variations in systemic exposure. SCID mice bearing NB1691 xenografts did not respond to oral topotecan $2 \mathrm{mg} / \mathrm{kg}$ (Chris Morton, personal communication May 30, 2006). In SCID mice the topotecan systemic exposure after an oral dose of $2 \mathrm{mg} / \mathrm{kg}$ is $55 \mathrm{ng} / \mathrm{mL} * \mathrm{hr}$ (136), approximately equivalent to that achieved by $0.36 \mathrm{mg} / \mathrm{kg}$ i.v. topotecan. In CBA/CaJ mice bearing NB1691 xenografts, a partial response was achieved in mice treated with $0.36 \mathrm{mg} / \mathrm{kg}$ i.v. topotecan, indicating that variations in mouse strain may impact the response of NB1691 xenografts to topotecan.

The NB1691 cell line used to establish xenografts had been maintained in tissue culture. Cell lines maintained in tissue culture are subject to alterations (175) that may influence sensitivity to cytotoxic drugs including topotecan. For example, promoter methylation and silencing of apoptosis-related genes can occur during passage in tissue culture (176). Genetic instability including chromosomal gains, losses, or translocation and gene amplification or deletion can also accumulate during tissue culture passage (176). Enhanced recombinatorial repair of topotecan-induced DNA strand breaks has been implicated in camptothecin analog resistance and may occur during passage over time in culture (177). Inhibition of cell death signaled by topotecan-induced DNA lesions may also result in topotecan resistance. Alternatively, enhanced activity of the DNA repair enzyme MGMT can result in topotecan resistance (178). Any of these alterations may account for the failure to respond to topotecan in vivo.

Despite the decreased sensitivity of NB1691 xenografts in SCID mice, we predicted that xenografts derived from the MRP4 shRNA clones would be more sensitive 
to topotecan than the parental cell line. However, we did not observe differences in vivo response based on MRP4 expression.

Failure of the MRP4 shRNA may be attributed to retroviral vector silencing. Gene expression has been silenced in retroviral vectors containing the MLV LTR. This silencing is mediated by the binding of ELP, an embryonal LTR-binding protein that suppresses transcription of the MLV LTR. However, expression of the MRP4 shRNA cassette in the MSCV retroviral vector that we used was directed by the PCMV LTR in which the ELP binding site has been deleted, decreasing the potential for retroviral vector silencing $(160,179)$. Furthermore, GFP expression in all of the transduced xenografts was visualized with fluorescence microscopy, suggesting that the retroviral vector was not silenced in vivo.

The disparity in the effect of MRP4 expression on topotecan sensitivity observed in vitro and in vivo may also be due in part to differences in the in vitro and in vivo environments. For example, Mattern et al demonstrated that resistance conferred by ABC transporters in vitro was not be recapitulated in vivo for the camptothecin analogs (73). Pgp expression in vitro conferred 15-35 fold resistance to topotecan. In contrast, Pgp expression in vivo did not significantly alter topotecan sensitivity.

The hypoxic tumor microenvironment may contribute to the disparity between in vitro and in vivo sensitivity to topotecan observed in our studies. MRP4 expression may have been induced in the hypoxic tumor microenvironment, overwhelming any RNAimediated knockdown in MRP4 expression. Recent in vitro studies have demonstrated increased MRP4 mRNA expression in NB1691 after 24 hours of hypoxia (Dr. Stacy Throm, personal communication March 14, 2007). BCRP expression is also induced 
under hypoxia via the HIF-1 pathway in placental choriocarcinoma, osteosarcoma, and acute myelogenous leukemia cell lines (180). The hypoxic tumor microenvironment stands in contrast to the oxygen replete in vitro environment that permits persistent MRP4 knockdown and differential topotecan sensitivity based on MRP4 expression.

The results of these studies highlight the differences between in vitro and in vivo environments. While we observed shRNA mediated MRP4 reduction in vitro that correlated with increased sensitivity to the camptothecin analogs, the same patterns were not recapitulated in the xenograft models.

Although the goal of these experiments was to determine the effect of MRP4 expression in vivo on the response of neuroblastoma to topotecan, the results do not provide an answer to this research question. However, because all of the NB1691 clones studied herein expressed MRP4 and failed to respond to topotecan, the results of these studies are consistent with the hypothesis that MRP4 expression confers resistance to topotecan and may be a factor in failure of some neuroblastomas to respond. Future studies in which MRP4 can be effectively silenced in vivo will be required to address this issue definitively. 


\section{Chapter 4. ABC transporter expression in neuroblastoma biopsy specimens from children treated with pharmacokinetically guided topotecan}

\subsection{Introduction}

The camptothecin analog topotecan is active against neuroblastoma in preclinical xenograft models. Based on the preclinical activity of topotecan in models of childhood solid tumors, topotecan has been evaluated in phase I and II clinical trials in children. In a phase II trial of topotecan in 30 children with high-risk neuroblastoma, a complete response was observed in 1 child, and partial responses were observed in 17 children for a response rate of $60 \%$ (15). Despite this favorable response to topotecan, a higher response rate was predicted based upon the anti-tumor activity of topotecan in neuroblastoma xenograft models. The $40 \%$ of children with high-risk neuroblastoma who did not respond to topotecan represent a subset of the population resistant to topotecan.

We have also demonstrated in neuroblastoma cell lines in vitro that the $\mathrm{ABC}$ transporters MRP4 and Pgp confer topotecan resistance. Thus, the objective of the current study was to evaluate the potential relation between $\mathrm{ABC}$ transport protein expression and clinical response to topotecan. Therefore, we evaluated the expression of $\mathrm{ABC}$ transporters for which topotecan is a substrate (i.e., MRP4, Pgp, and BCRP) in tumor specimens from children enrolled on the phase II trial of topotecan in high-risk neuroblastoma. We used immunohistochemistry to determine the plasma membrane expression of $\mathrm{ABC}$ transport proteins in neuroblastoma samples. 


\subsection{Materials and methods}

\subsubsection{Patients}

All patients evaluated in this retrospective study were enrolled on a phase II trial of pharmacokinetically guided topotecan at St. Jude Children's Research Hospital (15). Patients enrolled on the phase II trial had previously untreated high-risk neuroblastoma (i.e., INSS stage 3 or 4 disease in children older than 1 year or MYCN amplified stage 3 or 4 disease in children younger than 1 year). The St. Jude Institutional Review Board approved this retrospective study.

\subsubsection{Tumor specimens}

We attempted to evaluate $\mathrm{ABC}$ transporter expression in bone marrow aspirates obtained prior to therapy. However, the acid decalcification of these specimens prevented effective antigen retrieval and immunohistochemical detection of the proteins of interest. Therefore, we used formalin-fixed paraffin-embedded tumor specimens obtained during resection of the primary tumor after two cycles of topotecan and standard induction therapy including cyclophosphamide, doxorubicin, etoposide, cisplatin, ifosfamide, and carboplatin.

\subsubsection{Definitions of response to pharmacokinetically guided topotecan}

Response to pharmacokinetically guided topotecan was evaluated after two cycles and defined by extent of tumor regression. A complete response (CR) was more than $90 \%$ regression of the primary tumor and complete resolution of metastatic disease. A partial response (PR) was more than 50\% regression of all disease. An objective response 
(OR) was $25-50 \%$ tumor regression. Stable disease (SD) was less than $25 \%$ tumor regression without tumor progression. Patients with a CR or PR were classified as responders, and those with OR or SD were classified as non-responders. Standard radiographic methods (e.g., CT scans, MRI scans) were used to evaluate response.

\subsubsection{Immunohistochemistry}

Sections $(4 \mu \mathrm{m})$ of formalin-fixed paraffin-embedded neuroblastoma were placed on positively charged slides. Slides were heated $\left(60^{\circ} \mathrm{C}\right)$ for 30 minutes and cleared of paraffin in CitriSolv (Fisher Scientific, Hampton, NH) and alcohol gradient solution panel prior to high temperature antigen recovery in citrate buffer $\mathrm{pH}$ 6.0. Slides were blocked with 3\% hydrogen peroxide in methanol and avidin/ biotin blocking reagent (Vector Labs, Burlingame CA) prior to incubation with the primary antibody. MRP4 was probed with the rat anti-human monoclonal antibody $\mathrm{M}_{4} \mathrm{I}-10$ (Axxora). BCRP was probed with the rat anti-human and mouse monoclonal antibody BXP-53 (Axxora). Pgp was probed with the mouse anti-human monoclonal antibody JSB-1 (Signet Labs). Irrelevant isotype fractions were used as negative controls. Biotinylated secondary antibodies were used (Vector Labs), and the tertiary reagent was streptavidin-horseradish peroxidase (Dako, Carpinteria, CA). Color development was achieved with DAB (Dako), and the slides were counterstained with hematoxylin (Dako). Human kidney sections were used as positive controls for MRP4, Pgp, and BCRP staining.

Stained slides were visualized under a light microscope at 40x magnification. Only tumors with membrane staining of tumor cells were considered positive for expression of the individual transporters. Staining of endothelial cells within the tumor was not scored as positive. 


\subsubsection{Statistical considerations}

The Fisher exact test was used to determine if the children for whom tumor samples were available were representative of the study population in terms of response to topotecan. The Fisher exact test was also used to determine if $\mathrm{ABC}$ transporter expression was a significant indicator of response to topotecan. All tests were two-tailed.

\subsection{Results}

Neuroblastoma sections were available from 14 out of 28 evaluable patients. The positive and negative controls in human kidney are depicted in Figure 4.1. MRP4, Pgp, and BCRP are expressed in the brush border of the proximal convoluted tubule. No staining was noted in the isotype negative controls.

Out of the 14 available neuroblastoma sections, MRP4 was expressed in 2, Pgp was expressed in 4, and BCRP was expressed in 9 (Table 4.1). Neuroblastomas from four of eight responders expressed at least one of the $\mathrm{ABC}$ transporters evaluated. Tumors from four of the six non-responders expressed at least one of the $\mathrm{ABC}$ transporters evaluated. The neuroblastoma section from patient nine was representative of all specimens in which MRP4 was not expressed (Figure 4.2A). MRP4 was expressed in the membrane of neuroblastoma cells from patient number seven (Figure 4.2B). Pgp was not expressed in the neuroblastoma specimen from patient 13 (Figure 4.2C). Pgp was localized to the plasma membrane of neuroblastoma cells from patient number ten (Figure 4.2D), but was heterogeneous and localized to only a few regions of the tumor specimen. This pattern of sporadic Pgp staining was representative of all Pgp positive specimens. BCRP was not expressed in the neuroblastoma cells from patient three (Figure 4.2E). The tumor from patient two expressed BCRP (Figure 4.2F). 

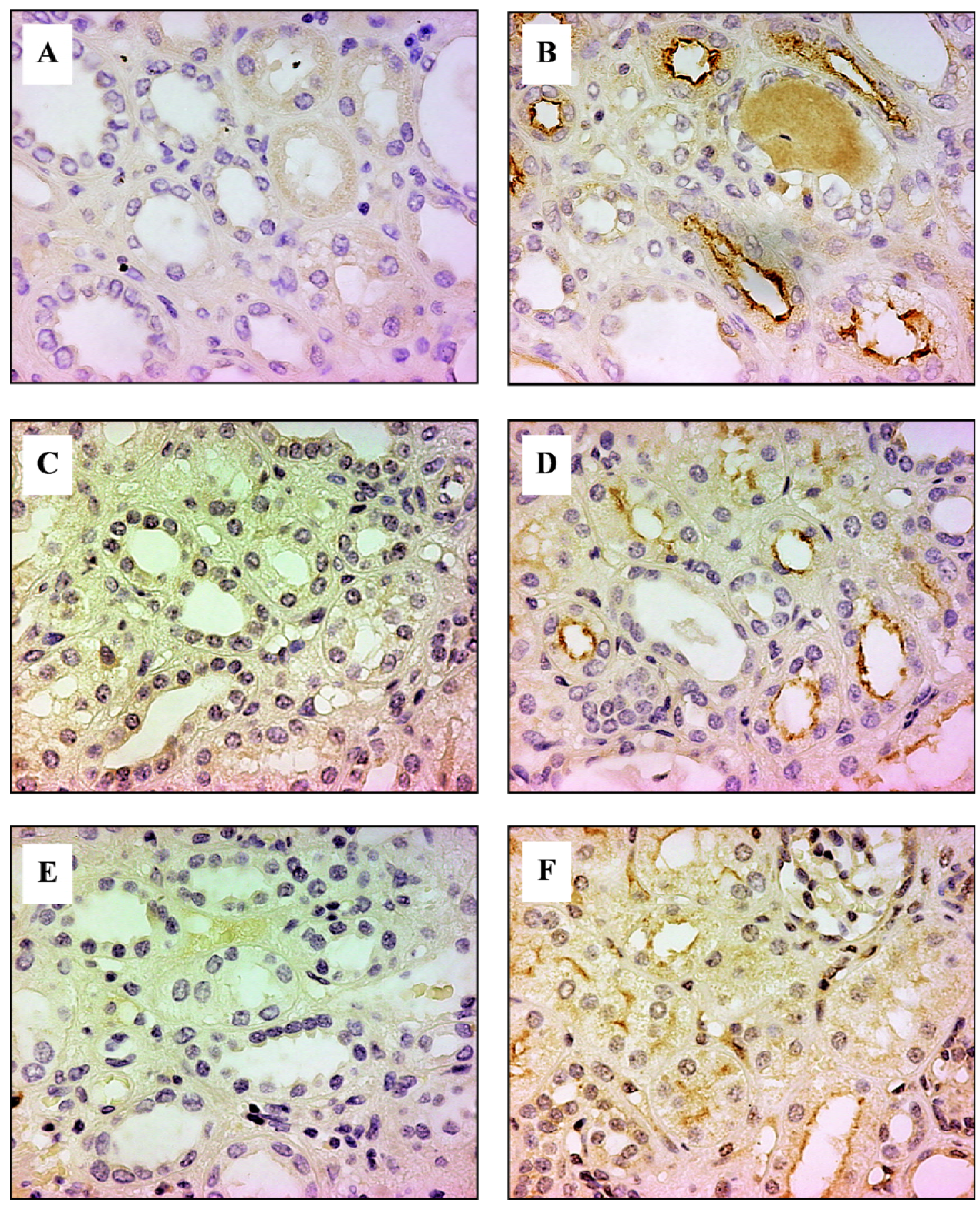

Figure 4.1 ABC transporter expression in human kidney controls.

Left column: irrelevant IgG negative control. Right column: positive control. Rat IgG (A); M $\mathrm{M}_{4} \mathrm{I}-10$ (B); Mouse IgG (C); JSB-1 (D); Rat IgG (E); BXP-53 (F). Brown staining in panels B, D, and F indicate localization of MRP4, Pgp, and BCRP to brush border of the proximal tubules. All images are shown at 40x magnification. 
Table 4.1 ABC transporter expression in neuroblastoma specimens and response to pharmacokinetically guided topotecan.

\begin{tabular}{ccccc}
\hline Patient Number & Response & $\begin{array}{c}\text { MRP4 } \\
\text { expression }\end{array}$ & $\begin{array}{c}\text { Pgp } \\
\text { expression }\end{array}$ & $\begin{array}{c}\text { BCRP } \\
\text { expression }\end{array}$ \\
\hline 1 & PR & - & - & - \\
2 & PR & - & - & + \\
3 & PR & - & - & - \\
4 & PR & - & - & + \\
5 & PR & - & + & + \\
6 & PR & - & + & + \\
7 & PR & + & - & + \\
8 & PR & + & - & - \\
9 & OR & - & - & + \\
10 & OR & - & + & + \\
11 & SD & - & - & - \\
12 & SD & - & - & + \\
13 & SD & - & - & - \\
14 & SD & - & + & + \\
\hline
\end{tabular}

Abbreviations: MRP4- multi-drug resistance associated protein 4; Pgp- P-glycoprotein; BCRP- breast cancer resistance protein; PR-partial response; OR- objective response; SD- stable disease 

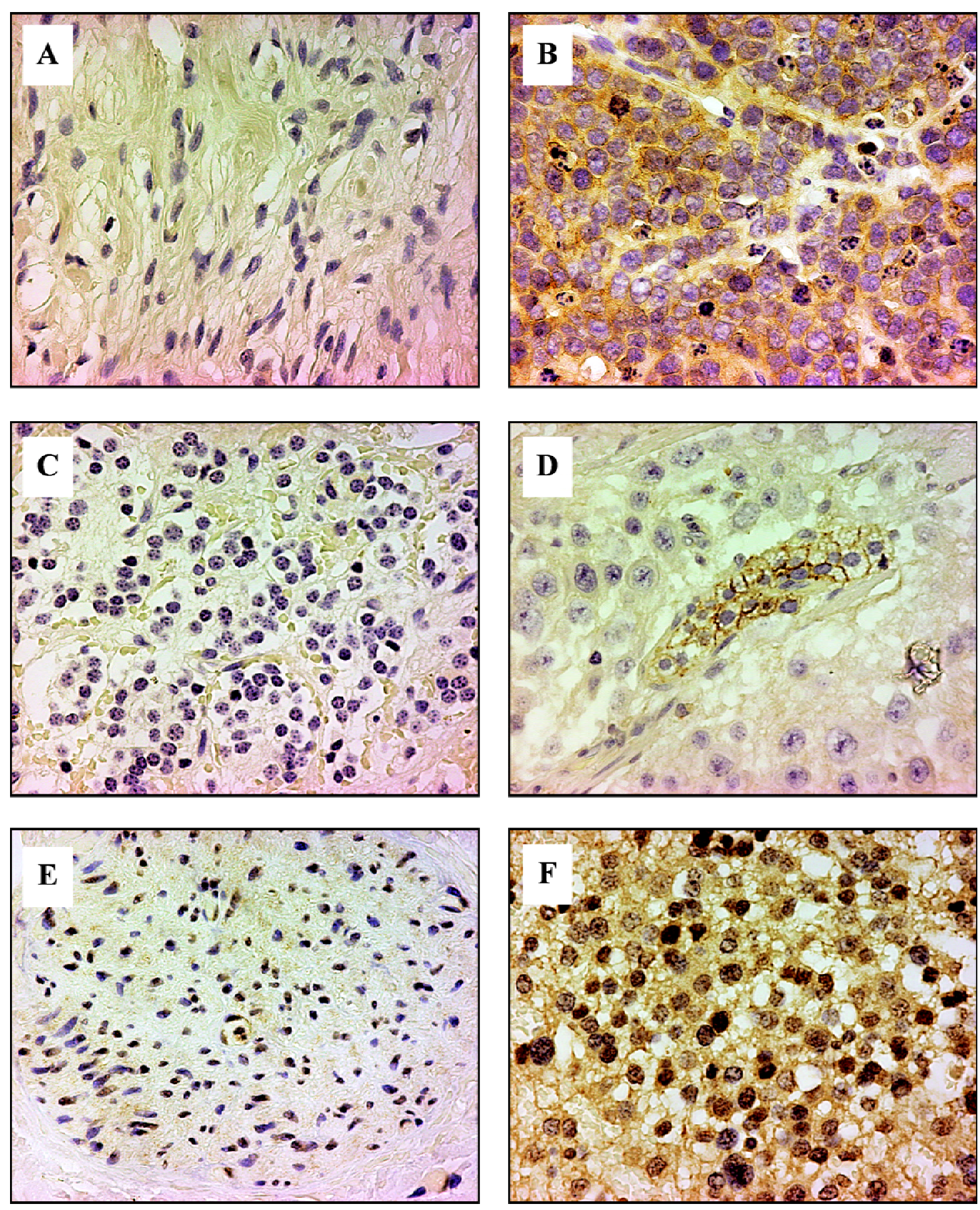

Figure 4.2 ABC transporter expression in neuroblastoma tissue obtained during primary tumor resection.

Patient 9: MRP4 negative (A); Patient 7: MRP4 positive (B); Patient 13: Pgp negative (C); Patient 10: Pgp positive (D); Patient 3: BCRP negative (E); Patient 2: BCRP positive (F). All images are shown at 40x magnification. 
Despite expression of MRP4, Pgp, or BCRP in neuroblastoma, these ABC transporters were not statistically related to response to pharmacokinetically guided topotecan $(P>0.05$, Fisher Exact test). There was an essentially even distribution of ABC transporter protein expression among responders and non-responders to topotecan. However, this was a small sample size and may not be sufficiently powered to detect a difference in $\mathrm{ABC}$ transporter expression between responders and non-responders.

\subsection{Discussion}

We used immunohistochemistry to detect the expression of MRP4, Pgp, and BCRP in primary neuroblastoma sections and determined the relation to topotecan response. When compared to methods such as RT-PCR to detect mRNA expression $(10,11,12,110)$ this immunohistochemical approach prevented contamination of $\mathrm{ABC}$ transporter expression in normal cells (e.g., vascular endothelium) that may obscure results. This immunohistochemical approach also permitted detection of $\mathrm{ABC}$ transporters localized to the plasma membrane of neoplastic cells

Based on the possibility of chemotherapy related induction or selection of $\mathrm{ABC}$ transporters, we initially attempted to determine the $\mathrm{ABC}$ transporter expression profile in bone marrow aspirates obtained prior to therapy. However, acid decalcification of the bone marrow aspirates prevented antigen retrieval and detection of $\mathrm{ABC}$ transporter expression. Due to the availability of formalin-fixed, paraffin-embedded primary tumor tissue we decided to evaluate the $\mathrm{ABC}$ transporter expression in these samples. However, these tumors were resected after two topotecan cycles and standard multi-agent induction therapy. In these available samples, expression of MRP4, Pgp, or BCRP was not related to failure to respond to topotecan. 
Previous studies have demonstrated that high levels of Pgp and MRP4 mRNA expression are predictive of poor outcome in neuroblastoma. Several factors may explain why a relation between MRP4/Pgp/BCRP expression and the clinical response to pharmacokinetically guided topotecan was not detected in the current study. The $\mathrm{ABC}$ transporter expression profile in the primary tumor may have changed as a result of induction therapy. Whereas cyclophosphamide, doxorubicin, and etoposide may be subject to $\mathrm{ABC}$ transporter mediated resistance, cisplatin, ifosfamide, and carboplatin may have eliminated the MRP4/Pgp/BCRP expressing clones responsible for failure to respond to topotecan. Although this phenomenon is the rationale for multi-agent chemotherapy, exposure of the neuroblastoma specimens to these drugs may have confounded our results.

Norris and colleagues demonstrated MRP4 mRNA expression in $100 \%$ of 52 neuroblastomas from untreated patients (110). In this population of 52 patients, high MRP4 mRNA expression was a significant indicator of poor survival. The previous studies demonstrating a relation between $\mathrm{ABC}$ transporter expression and outcome evaluated mRNA, not protein expression $(11,12,10)$. Although the real-time RT-PCR method permits simple and rapid quantitation of mRNA expression in tumor homogenate, this method does not necessarily isolate tumor tissue from normal tissue such as blood vessels. This is particularly problematic for the $\mathrm{ABC}$ transporters, which are expressed in vascular endothelial cells. Furthermore, the tissue must be preserved in order to isolate high quality RNA, and a technique such as laser capture microdissection should be used to isolate neuroblastoma cells from endothelia (181). Immunohistochemistry permits detection of $\mathrm{ABC}$ transporter subcellular localization. Cytosolic localization of a 
transporter would not likely result in resistance because the transporter is not oriented to efflux substrates from the cell.

Whether response to topotecan predicts long-term outcome such as overall survival or event-free-survival remains to be determined. Evaluating whether the $\mathrm{ABC}$ transporter protein expression profile in the primary or metastatic tumor at any point (before, during, or after therapy) predicts outcome may help to improve the response of neuroblastoma to the camptothecin analogs. To accomplish this in a clinical study of children with neuroblastoma, the question about correlation between $\mathrm{ABC}$ transporter mRNA and protein expression must first be addressed. ABC transporter expression in the same tumor sample should be evaluated using both real-time RT-PCR and immunohistochemistry. Next, ABC transporter expression should be evaluated before and after therapy to determine the influence of chemotherapy on $\mathrm{ABC}$ transporter expression. Finally, a clinical study should be sufficiently powered to determine the relationship between $\mathrm{ABC}$ transporter expression and clinical outcome in children with neuroblastoma. 


\section{Chapter 5. Summary and future directions}

Neuroblastoma is the most common extracranial solid tumor in children. Patients who present with localized disease have excellent predicted survival. However, most children diagnosed with neuroblastoma are at high-risk for a poor outcome because they present with metastatic disease and/ or other unfavorable biological features including MYCN amplification. Therapy for children who are diagnosed with high-risk disease can include multi-agent chemotherapy, myeloablation with bone marrow transplant/ stem cell rescue, surgery, and radiation. Despite this intense therapy, five-year survival in children with high-risk neuroblastoma is much less favorable than for those with low- or intermediate-risk disease ( 30 to $40 \%$ compared to 80 to $95 \%$ ).

One of the approaches recently evaluated for improving the outcome in children with high-risk neuroblastoma is pharmacokinetically guided topotecan dosing $(49,15)$. This approach was based on significant anti-tumor activity of topotecan systemic exposures of $100 \mathrm{ng} / \mathrm{mL}^{*} \mathrm{hr}$ in neuroblastoma xenograft models. Because topotecan clearance in children varies at least 10 -fold and in animal models topotecan anti-tumor activity depends on systemic exposure, topotecan dosage was individualized to achieve a targeted systemic exposure of 80 to $120 \mathrm{ng} / \mathrm{mL}^{*} \mathrm{hr}$ in children with newly diagnosed high-risk neuroblastoma. Pharmacokinetically guided topotecan dosing was feasible in this population, and the response to this single agent was considered excellent. However, a significant proportion of children $(40 \%)$ did not respond to topotecan and represent a subset of the disease that is resistant to topotecan. 
$\mathrm{ABC}$ transporter mediated efflux of topotecan from neoplastic cells has been implicated in topotecan resistance. The $\mathrm{ABC}$ transporters for which topotecan is a substrate include Pgp, MRP1, MRP4, and BCRP. ABC transporters also confer resistance to other cytotoxic drugs traditionally used in high-risk neuroblastoma therapy (i.e., doxorubicin, etoposide, and vincristine). Furthermore, MRP1, Pgp, and MRP4 are expressed in neuroblastoma, and high levels of mRNA expression of these transporters is predictive of poor overall survival and event-free survival $(10,11,12,110)$. However, the relation between expression of $\mathrm{ABC}$ transport proteins in neuroblastoma cells and antitumor response to $\mathrm{ABC}$ transporter substrates has not been evaluated.

The major objective of these studies was to evaluate the relation between expression of $\mathrm{ABC}$ transport proteins in neuroblastoma and response to the camptothecin analogs topotecan and $\mathrm{SN}-38$. The first specific aim was to determine the contribution of $\mathrm{ABC}$ transporters to topotecan and irinotecan/SN-38 sensitivity in neuroblastoma cell lines. After identifying $\mathrm{ABC}$ transporters that confer resistance to camptothecin analogs in vitro, we evaluated the contribution of $\mathrm{ABC}$ transporter expression in neuroblastoma xenografts to topotecan response in vivo. Finally, we assessed the relation between expression of $\mathrm{ABC}$ transporters in neuroblastoma biopsy specimens and the anti-tumor response to pharmacokinetically guided topotecan.

First, by western blot analysis we screened a panel of neuroblastoma cell lines with a range of sensitivity to topotecan for expression of ABC transporters. NB1691, the most topotecan resistant cell line evaluated, expressed both MRP4 and Pgp. In contrast, NB1643, the most topotecan sensitive cell line evaluated, expressed neither MRP4 nor Pgp. Based upon these results, we determined the effect of reducing MRP4 and Pgp 
expression via shRNA in NB1691 on topotecan and SN-38 sensitivity. As expected, topotecan sensitivity increased in NB1691 clones with reduced MRP4 and Pgp protein expression. We also demonstrated that topotecan sensitivity was reduced upon overexpression of MRP4 in NB1643.

To determine if the increased in vitro topotecan sensitivity in NB1691 MRP4 shRNA clones translated into increased sensitivity in vivo, we established these NB1691 clones as xenografts in SCID mice. Mice bearing neuroblastoma xenografts were treated with topotecan at dosages that had been shown to elicit at least $50 \%$ tumor volume regression in NB1691 xenografts (155). Unexpectedly, no tumor regression was observed in any of the xenografts evaluated. Furthermore, no difference was noted in response to topotecan between the parental NB1691 and the MRP4 shRNA clones. Most likely this occurred because MRP4 knock down did not persist in vivo.

Because the rationale for this study of $\mathrm{ABC}$ transporters in camptothecin analog resistance was based upon the failure to respond to topotecan in $40 \%$ of children with high-risk neuroblastoma in a phase II trial, we also retrospectively evaluated the $\mathrm{ABC}$ transporter expression profile in neuroblastoma specimens from that phase II trial. Based on the in vitro data demonstrating increased sensitivity to topotecan in neuroblastoma clones with reduced MRP4 and Pgp expression, we reasoned that MRP4 and/ or Pgp would be expressed in neuroblastoma tissue from children who did not respond to topotecan. However, results of immunohistochemical detection of $\mathrm{ABC}$ transport protein in the membrane of neuroblastoma cells demonstrated that expression of MRP4, Pgp, or BCRP was not related to response to topotecan. Because this analysis could not be conducted on tissue prior to therapy, the results may be confounded by the effects of 
multi-agent chemotherapy. Including tumor samples obtained prior to chemotherapy and preserved to ensure optimal antigen recovery in future clinical studies of neuroblastoma may permit a broader assessment of the impact of $\mathrm{ABC}$ transporter expression on antitumor activity of drugs.

The findings from the in vitro and xenograft studies conflict with the results of evaluation of clinical samples from children with neuroblastoma. The in vitro studies demonstrated that MRP4 and Pgp confer resistance to topotecan. The xenograft studies pointed towards a potential association between MRP4 expression and failure to respond to topotecan. But the lack of association between $\mathrm{ABC}$ transporter expression in the clinical samples and response to topotecan may be confounded by the timing of sample acquisition after two topotecan courses and induction therapy. More than highlighting the limitations of experimental models of neuroblastoma, the implications of our results also highlight the importance of determining $\mathrm{ABC}$ transporter expression prior to potential clonal selection by multi-agent chemotherapy in children with neuroblastoma. However, further preclinical xenograft studies and clinical studies will have to be conducted to determine the contribution of $\mathrm{ABC}$ transporter protein expression to response to individual drugs and overall outcome in neuroblastoma.

The $\mathrm{ABC}$ transporter superfamily is only one class of proteins that influences anti-tumor response to drugs used in neuroblastoma therapy. Novel determinants of response could also be identified using mRNA gene expression arrays or single nucleotide polymorphism (SNP) chip arrays on laser capture microdissected tumor tissue. If tumor samples can be obtained at discrete time points during therapy including at diagnosis, after induction, consolidation, and bone marrow transplant, therapy could 
potentially be individualized and optimized based upon changes in gene expression signature.

Further preclinical studies could also be conducted to assess the effect of ABC transporter expression on anti-tumor response in neuroblastoma. For example, a mouse model of neuroblastoma could be developed to address the contribution of $\mathrm{ABC}$ transporters to tumor pathology and response to drugs. To accomplish this, the MRP4 deficient mouse (114), for example, could be crossed with the transgenic mouse model overexpressing MYCN in neuroectodermal cells(182). Other ABC transporter knock out models including Bcrp and Mdrla/1b models could also be crossed with the MYCN transgenic mouse. If the progeny of these crosses were viable, neuroblastoma development could be compared to the parental MYCN transgenic mouse to assess the role of $\mathrm{ABC}$ transporters in tumor formation. If tumors form in the progeny, they could be treated with various drugs including topotecan and irinotecan to determine the effect of $\mathrm{ABC}$ transporters on tumor response. But there is a caveat that the absence of an $\mathrm{ABC}$ transporter in normal tissues such as the kidneys will alter the pharmacokinetics of the drug. So an appropriate dosage that yields equivalent systemic exposures in the MYCN transgenic mouse and the MRP4 knockout/ MYCN transgenic progeny must be determined empirically.

As $\mathrm{ABC}$ transporter expression is upregulated by hypoxia in osteosarcoma, acute myelogenous leukemia, and choricarcinoma cell lines(180), the role of hypoxia in resistance to topotecan and irinotecan should be assessed. In future xenograft studies, administration of RNAi in vivo (183) may permit more precise control over reduction of $\mathrm{ABC}$ transporter expression in the hypoxic tumor microenvironment. 
Pharmacokinetically guided topotecan in combination with other agents (e.g., etoposide (184), cyclophosphamide (3), vincristine (185), and gefitinib $(130,136))$ may be an effective approach to improve long-term survival in high-risk neuroblastoma and other pediatric cancers. In evaluating these combinations, it will be important to use appropriate drug sequences, schedules, dosages, and systemic exposures in order to minimize host toxicity and maximize anti-tumor response. Additional analysis of the role of $\mathrm{ABC}$ transport protein expression in clinical response to these combinations may further improve the utility of the camptothecin analogs in neuroblastoma.

In conclusion, our studies show that MRP4 and Pgp confer resistance to topotecan and SN38 in neuroblastoma. We developed an RNAi approach to silencing the expression of these $\mathrm{ABC}$ transporters in neuroblastoma to determine the contribution to camptothecin analog resistance. Future studies in children with neuroblastoma will further delineate the clinical relevance of our findings. The application of these data in the care of children with neuroblastoma may ultimately improve clinical outcome. 
List of References 
1. Grovas A, Fremgen A, Rauck A, Ruymann FB, et al. The National Cancer Data Base report on patterns of childhood cancers in the United States, Cancer, 80: 2321-2332, 1997.

2. Castleberry RP. Neuroblastoma, Eur J Cancer, 33: 1430-1437, 1997.

3. Kretschmar CS, Kletzel M, Murray K, Thorner P, et al. Response to paclitaxel, topotecan, and topotecan-cyclophosphamide in children with untreated disseminated neuroblastoma treated in an upfront phase II investigational window: a pediatric oncology group study, J Clin Oncol, 22: 4119-4126, 2004.

4. Ireland CM, Pittman SM, Jones SL, and Harnett PR. Establishment of an in vitro model for cisplatin resistance in human neuroblastoma cell lines, Anticancer Res, 14: 2397-2403, 1994.

5. Timmer-Bosscha H, Mulder NH, and de Vries EG. Modulation of cisdiamminedichloroplatinum(II) resistance: a review, Br J Cancer, 66: 227-238, 1992.

6. Yasuno T, Matsumura $\mathrm{T}$, Shikata $\mathrm{T}$, Inazawa $\mathrm{J}$, et al. Establishment and characterization of a cisplatin-resistant human neuroblastoma cell line, Anticancer Res, 19: 4049-4057, 1999.

7. Ho R, Eggert A, Hishiki T, Minturn JE, et al. Resistance to chemotherapy mediated by TrkB in neuroblastomas, Cancer Res, 62: 6462-6466, 2002.

8. Hetman M, Kanning K, Cavanaugh JE, and Xia Z. Neuroprotection by brainderived neurotrophic factor is mediated by extracellular signal-regulated kinase and phosphatidylinositol 3-kinase, J Biol Chem, 274: 22569-22580, 1999.

9. Lee WP. Purification and characterization of tubulin from parental and vincristine-resistant HOB1 lymphoma cells, Arch Biochem Biophys, 319: 498503, 1995.

10. Norris MD, Bordow SB, Marshall GM, Haber PS, et al. Expression of the gene for multidrug-resistance-associated protein and outcome in patients with neuroblastoma, N Engl J Med, 334: 231-238, 1996.

11. Haber M, Bordow SB, Haber PS, Marshall GM, et al. The prognostic value of MDR1 gene expression in primary untreated neuroblastoma, Eur J Cancer, 33: 2031-2036, 1997.

12. Haber M, Smith J, Bordow SB, Flemming C, et al. Association of high-level MRP1 expression with poor clinical outcome in a large prospective study of primary neuroblastoma, J Clin Oncol, 24: 1546-1553, 2006. 
13. Seeger RC, Brodeur GM, Sather H, Dalton A, et al. Association of multiple copies of the N-myc oncogene with rapid progression of neuroblastomas, N Engl J Med, 313: 1111-1116, 1985.

14. Manohar CF, Bray JA, Salwen HR, Madafiglio J, et al. MYCN-mediated regulation of the MRP1 promoter in human neuroblastoma, Oncogene, 23: 753$762,2004$.

15. Santana VM, Furman WL, Billups CA, Hoffer F, et al. Improved response in high-risk neuroblastoma with protracted topotecan administration using a pharmacokinetically guided dosing approach, J Clin Oncol, 23: 4039-4047, 2005.

16. Kushner BH, Kramer K, LaQuaglia MP, Modak S, et al. Reduction from seven to five cycles of intensive induction chemotherapy in children with high-risk neuroblastoma, J Clin Oncol, 22: 4888-4892, 2004.

17. Furman WL, Stewart CF, Poquette CA, Pratt CB, et al. Direct translation of a protracted irinotecan schedule from a xenograft model to a phase I trial in children, J Clin Oncol, 17: 1815-1824, 1999.

18. Litman T, Druley TE, Stein WD, and Bates SE. From MDR to MXR: new understanding of multidrug resistance systems, their properties and clinical significance, Cell and Molecular Life Sciences, 58: 931-959, 2001.

19. Vasilatou-Kosmidis H. Cancer in neonates and infants, Med Pediatr Oncol, 41: 79, 2003.

20. Gurney JG, Severson RK, Davis S, and Robison LL. Incidence of cancer in children in the United States. Sex-, race-, and 1-yr. age-specific rates by histologic groups, Cancer, 75: 2186-2195, 1995.

21. Brodeur GM and Castleberry RP. Neuroblastoma. In: Pizzo PA and Poplack DG (eds.), Principles and Practice of Pediatric Oncology, pp. 761-789. LippincottRaven Publishers: Philadelphia, PA, 1997.

22. Escobar MA, Grosfeld JL, Powell RL, West KW, et al. Long-term outcomes in patients with stage IV neuroblastoma, J Pediatr Surg, 41: 377-381, 2006.

23. Ilias I and Pacak K. Diagnosis and management of tumors of the adrenal medulla, Horm Metab Res, 37: 717-721, 2005.

24. Brodeur GM, Seeger RC, Barrett A, Berthold F, et al. International criteria for diagnosis, staging, and response to treatment in patients with neuroblastoma, $\mathrm{J}$ Clin Oncol, 6: 1874-1881, 1988.

25. Brodeur GM, Pritchard J, Berthold F, Carlsen NLT, et al. Revisions of the international criteria for neuroblastoma diagnosis, staging, and response to treatment [see comments], J Clin Oncol, 11: 1466-1477, 1993. 
26. Evans AE and D'Angio GJ. Age at diagnosis and prognosis in children with neuroblastoma, J Clin Oncol, 23: 6443-6444, 2005.

27. London WB, Castleberry RP, Matthay KK, Look AT, et al. Evidence for an age cutoff greater than 365 days for neuroblastoma risk group stratification in the Children's Oncology Group, J Clin Oncol, 23: 6459-6465, 2005.

28. George RE, London WB, Cohn SL, Maris JM, et al. Hyperdiploidy plus nonamplified MYCN confers a favorable prognosis in children 12 to 18 months old with disseminated neuroblastoma: a Pediatric Oncology Group study, J Clin Oncol, 23: 6466-6473, 2005.

29. Schmidt ML, Lal A, Seeger RC, Maris JM, et al. Favorable prognosis for patients 12 to 18 months of age with stage 4 nonamplified MYCN neuroblastoma: a Children's Cancer Group Study, J Clin Oncol, 23: 6474-6480, 2005.

30. Schwab M, Alitalo K, Klempnauer KH, Varmus HE, et al. Amplified DNA with limited homology to myc cellular oncogene is shared by human neuroblastoma cell lines and a neuroblastoma tumour, Nature, 305: 245-248, 1983.

31. Lee WH, Murphree AL, and Benedict WF. Expression and amplification of the N-myc gene in primary retinoblastoma, Nature, 309: 458-460, 1984.

32. Nau MM, Brooks BJ, Jr., Carney DN, Gazdar AF, et al. Human small-cell lung cancers show amplification and expression of the N-myc gene, Proc Natl Acad Sci U S A, 83: 1092-1096, 1986.

33. Grandori C and Eisenman RN. Myc target genes, Trends Biochem Sci, 22: 177$181,1997$.

34. Lutz W, Stohr M, Schurmann J, Wenzel A, et al. Conditional expression of Nmyc in human neuroblastoma cells increases expression of alpha-prothymosin and ornithine decarboxylase and accelerates progression into S-phase early after mitogenic stimulation of quiescent cells, Oncogene, 13: 803-812, 1996.

35. Goodman LA, Liu BC, Thiele CJ, Schmidt ML, et al. Modulation of N-myc expression alters the invasiveness of neuroblastoma, Clin Exp Metastasis, 15: 130-139, 1997.

36. Maris JM and Matthay KK. Molecular biology of neuroblastoma, J Clin Oncol, 17: 2264-2279, 1999.

37. Shimada H, Chatten J, Newton WA, Jr., Sachs N, et al. Histopathologic prognostic factors in neuroblastic tumors: definition of subtypes of ganglioneuroblastoma and an age-linked classification of neuroblastomas, J Natl Cancer Inst, 73: 405-416, 1984. 
38. Chatten J, Shimada H, Sather HN, Wong KY, et al. Prognostic value of histopathology in advanced neuroblastoma: a report from the Children's Cancer Study Group, Hum Pathol, 19: 1187-1198, 1988.

39. Shimada H, Umehara S, Monobe $\mathrm{Y}$, Hachitanda $\mathrm{Y}$, et al. International neuroblastoma pathology classification for prognostic evaluation of patients with peripheral neuroblastic tumors: a report from the Children's Cancer Group, Cancer, 92: 2451-2461, 2001.

40. Look AT, Hayes FA, Nitschke R, McWilliams NB, et al. Cellular DNA content as a predictor of response to chemotherapy in infants with unresectable neuroblastoma, N Engl J Med, 311: 231-235, 1984.

41. Gansler T, Chatten J, Varello M, Bunin GR, et al. Flow cytometric DNA analysis of neuroblastoma. Correlation with histology and clinical outcome, Cancer, 58: 2453-2458, 1986.

42. Taylor SR, Blatt J, Costantino JP, Roederer M, et al. Flow cytometric DNA analysis of neuroblastoma and ganglioneuroma. A 10-year retrospective study, Cancer, 62: 749-754, 1988.

43. Ladenstein R, Ambros IM, Potschger U, Amann G, et al. Prognostic significance of DNA di-tetraploidy in neuroblastoma, Med Pediatr Oncol, 36: 83-92, 2001.

44. Nitschke R, Smith EI, Shochat S, Altshuler G, et al. Localized neuroblastoma treated by surgery: a Pediatric Oncology Group Study, J Clin Oncol, 6: 12711279, 1988.

45. Kushner BH, LaQuaglia MP, Bonilla MA, Lindsley K, et al. Highly effective induction therapy for stage 4 neuroblastoma in children over 1 year of age, J Clin Oncol, 12: 2607-2613, 1994.

46. Goldie JH and Coldman AJ. A mathematic model for relating the drug sensitivity of tumors to their spontaneous mutation rate, Cancer Treat Rep, 63: 1727-1733, 1979 .

47. George RE, Li S, Medeiros-Nancarrow C, Neuberg D, et al. High-risk neuroblastoma treated with tandem autologous peripheral-blood stem cellsupported transplantation: long-term survival update, J Clin Oncol, 24: 28912896, 2006.

48. Matthay KK, Villablanca JG, Seeger RC, Stram DO, et al. Treatment of high-risk neuroblastoma with intensive chemotherapy, radiotherapy, autologous bone marrow transplantation, and 13-cis-retinoic acid. Children's Cancer Group, N Engl J Med, 341: 1165-1173, 1999. 
49. Santana VM, Zamboni WC, Kirstein MN, Tan M, et al. A pilot study of protracted topotecan dosing using a pharmacokinetically guided dosing approach in children with solid tumors, Clin Cancer Res, 9: 633-640, 2003.

50. Benedetti P, Fiorani P, Capuani L, and Wang JC. Camptothecin resistance from a single mutation changing glycine 363 of human DNA topoisomerase I to cysteine, Cancer Res, 53: 4343-4348, 1993.

51. Fujimori A, Harker WG, Kohlhagen G, Hoki Y, et al. Mutation at the catalytic site of topoisomerase I in $\mathrm{CEM} / \mathrm{C} 2$, a human leukemia cell line resistant to camptothecin, Cancer Res, 55: 1339-1346, 1995.

52. Dhooge CR, De Moerloose BM, Benoit YC, Van Roy N, et al. Expression of the MDR1 gene product P-glycoprotein in childhood neuroblastoma, Cancer, 80: 1250-1257, 1997.

53. de Cremoux P., Jourdan-Da-Silva N, Couturier J, Tran-Perennou C, et al. Role of chemotherapy resistance genes in outcome of neuroblastoma, Pediatr Blood Cancer, 48: 311-317, 2006.

54. Wall ME, Wani MC, Cook CE, and Palmer KH. Plant antitumor agents. I. The isolation and structure of camptothecin, a novel alkaloidal leukemia and tumor inhibitor from Camptotheca acuminata, J Am Chem Soc, 88: 3888-3890, 1966.

55. Gottlieb JA, Guarino AM, Call JB, Oliverio VT, et al. Preliminary pharmacologic and clinical evaluation of camptothecin sodium (NSC-100880), Cancer Chemother Rep, 54: 461-470, 1970.

56. Muggia FM, Creaven PJ, Hansen HH, Cohen MH, et al. Phase I clinical trial of weekly and daily treatment with camptothecin (NSC-100880): correlation with preclinical studies, Cancer Chemother Rep, 56: 515-521, 1972.

57. Moertel CG, Schutt AJ, Reitemeier RJ, and Hahn RG. Phase II study of camptothecin (NSC-100880) in the treatment of advanced gastrointestinal cancer, Cancer Chemother Rep, 56: 95-101, 1972.

58. Rivory LP and Robert J. Molecular, cellular, and clinical aspects of the pharmacology of 20(S)camptothecin and its derivatives, Pharmacol Ther, 68: 269296, 1995.

59. Hertzberg RP, Caranfa MJ, Holdern KG, Jakas DR, et al. Modification of the hydroxy lactone ring of camptothecin: inhibition of mammalian topoisomerase I and biological activity, Journal of Medicinal Chemistry, 32: 715-720, 1989.

60. Pommier Y. Topoisomerase I inhibitors: camptothecins and beyond, Nat Rev Cancer, 6: 789-802, 2006. 
61. Zamboni WC, Stewart CF, Thompson J, Santana VM, et al. Relationship between topotecan systemic exposure and tumor response in human neuroblastoma xenografts [see comments], J Natl Cancer Inst, 90: 505-511, 1998.

62. Houghton PJ, Cheshire PJ, Hallman JD, Lutz L, et al. Efficacy of topoisomerase I inhibitors, topotecan and irinotecan, administered at low dose levels in protracted schedules to mice bearing xenografts of human tumors, Cancer Chemother Pharmacol, 36: 393-403, 1995.

63. Zamboni WC, Houghton PJ, Hulstein JL, Kirstein M, et al. Relationship between tumor extracellular fluid exposure to topotecan and tumor response in human neuroblastoma xenograft and cell lines, Cancer Chemother Pharmacol, 43: 269276, 1999.

64. Pratt CB, Stewart CF, Santana VM, Bowman L, et al. Phase I study of topotecan for pediatric patients with malignant solid tumors, J Clin Oncol, 12: 539-543, 1994.

65. Blaney SM, Needle MN, Gillespie A, Sato JK, et al. Phase II trial of topotecan administered as 72-hour continuous infusion in children with refractory solid tumors: a collaborative Pediatric Branch, National Cancer Institute, and Children's Cancer Group Study, Clin Cancer Res, 4: 357-360, 1998.

66. Tubergen DG, Stewart CF, Pratt CB, Zamboni WC, et al. Phase I trial and pharmacokinetic (PK) and pharmacodynamics (PD) study of topotecan using a five-day course in children with refractory solid tumors: A Pediatric Oncology Group Study, J Ped Hem/Onc, 18: 352-361, 1996.

67. Langler A, Christaras A, Abshagen K, Krauth K, et al. Topotecan in the treatment of refractory neuroblastoma and other malignant tumors in childhood - a phase-IIstudy, Klin Padiatr, 214: 153-156, 2002.

68. Houghton PJ, Chesire PJ, Myers L, Stewart CF, et al. Evaluation of 9dimethylaminomethyl-10-hydroxycamptothecin against xenografts derived from adult and childhood solid tumors, Cancer Chemother Pharmacol, 31: 229-239, 1992.

69. Barthelmes HU, Habermeyer M, Christensen MO, Mielke C, et al. TDP1 overexpression in human cells counteracts DNA damage mediated by topoisomerases I and II, J Biol Chem, 279: 55618-55625, 2004.

70. Thompson J, Zamboni WC, Cheshire PJ, Lutz L, et al. Efficacy of systemic administration of irinotecan against neuroblastoma xenografts, Clin Cancer Res, 3: 423-431, 1997.

71. Thompson J, Zamboni WC, Cheshire PJ, Richmond LB, et al. Efficacy of oral administration of irinotecan against neuroblastoma xenografts., Anticancer Drugs, 8: 313-322, 1997. 
72. Vassal G, Terrier-Lacombe MJ, Bissery MC, Venuat AM, et al. Therapeutic activity of CPT-11, a DNA-topoisomerase I inhibitor, against peripheral primitive neuroectodermal tumour and neuroblastoma xenografts, Br J Cancer, 74: 537-545, 1996.

73. Mattern MR, Hofmann GA, Polsky RM, Funk LR, et al. In vitro and in vivo effects of clinically important camptothecin analogues on multidrug-resistant cells, Oncol Res, 5: 467-474, 1993.

74. Jansen WJ, Hulscher TM, Ark-Otte J, Giaccone G, et al. CPT-11 sensitivity in relation to the expression of $\mathrm{P} 170$-glycoprotein and multidrug resistanceassociated protein, Br J Cancer, 77: 359-365, 1998.

75. Houghton PJ, Stewart CF, Zamboni WC, Thompson J, et al. Schedule dependent efficacy of camptothecins in models of human cancer, New York Acad Sci, 803: 188-201, 1996.

76. Blaney S, Berg SL, Pratt C, Weitman S, et al. A phase I study of irinotecan in pediatric patients: a pediatric oncology group study, Clin Cancer Res, 7: 32-37, 2001.

77. Vassal G, Doz F, Frappaz D, Imadalou K, et al. A phase I study of irinotecan as a 3 -week schedule in children with refractory or recurrent solid tumors, J Clin Oncol, 21: 3844-3852, 2003.

78. Mugishima H, Matsunaga T, Yagi K, Asami K, et al. Phase I study of irinotecan in pediatric patients with malignant solid tumors, J Pediatr Hematol Oncol, 24: 94-100, 2002.

79. Dean M, Rzhetsky A, and Allikmets R. The human ATP-binding cassette (ABC) transporter superfamily, Genome Res, 11: 1156-1166, 2001.

80. Pleban K, Kopp S, Csaszar E, Peer M, et al. P-glycoprotein substrate binding domains are located at the transmembrane domain/transmembrane domain interfaces: a combined photoaffinity labeling-protein homology modeling approach, Mol Pharmacol, 67: 365-374, 2005.

81. Higgins $\mathrm{CF}$ and Gottesman MM. Is the multidrug transporter a flippase?, Trends Biochem Sci, 17: 18-21, 1992.

82. Gottesman MM. How cancer cells evade chemotherapy: sixteenth Richard and Hinda Rosenthal Foundation Award Lecture, Cancer Res, 53: 747-754, 1993.

83. Higgins $\mathrm{CF}$ and Linton KJ. The ATP switch model for ABC transporters, Nat Struct Mol Biol, 11: 918-926, 2004.

84. Juliano RL and Ling V. A surface glycoprotein modulating drug permeability in Chinese hamster ovary cell mutants, Biochim Biophys Acta, 455: 152-162, 1976. 
85. Molinari A, Calcabrini A, Meschini S, Stringaro A, et al. Subcellular detection and localization of the drug transporter P-glycoprotein in cultured tumor cells, Curr Protein Pept Sci, 3: 653-670, 2002.

86. Petriz J, Gottesman MM, and Aran JM. An MDR-EGFP gene fusion allows for direct cellular localization, function and stability assessment of P-glycoprotein, Curr Drug Deliv, 1: 43-56, 2004.

87. Gros P, Croop J, and Housman D. Mammalian multidrug resistance gene: complete cDNA sequence indicates strong homology to bacterial transport proteins, Cell, 47: 371-380, 1986.

88. van der Valk P, van Kalken CK, Ketelaars H, Broxterman HJ, et al. Distribution of multi-drug resistance-associated P-glycoprotein in normal and neoplastic human tissues. Analysis with 3 monoclonal antibodies recognizing different epitopes of the P-glycoprotein molecule, Ann Oncol, 1: 56-64, 1990.

89. Hendricks CB, Rowinsky EK, Grochow LB, Donehower RC, et al. Effect of Pglycoprotein expression on the accumulation and cytotoxicity of topotecan (SK\&F 104864), a new camptothecin analogue, Cancer Res, 52: 2268-2278, 1992.

90. Hoki Y, Fujimori A, and Pommier Y. Differential cytotoxicity of clinically important camptothecin derivatives in P-glycoprotein-overexpressing cell lines, Cancer Chemother Pharmacol, 40: 433-438, 1997.

91. Fojo AT, Ueda K, Slamon DJ, Poplack DG, et al. Expression of a multidrugresistance gene in human tumors and tissues, Proc Natl Acad Sci U S A, 84: 265269, 1987.

92. Goldstein LJ, Fojo AT, Ueda K, Crist W, et al. Expression of the multidrug resistance, MDR1, gene in neuroblastomas, J Clin Oncol, 8: 128-136, 1990.

93. Chan HS, Haddad G, Thorner PS, DeBoer G, et al. P-glycoprotein expression as a predictor of the outcome of therapy for neuroblastoma, N Engl J Med, 325: 16081614, 1991.

94. Chen G, Gharib TG, Huang CC, Taylor JM, et al. Discordant protein and mRNA expression in lung adenocarcinomas, Mol Cell Proteomics, 1: 304-313, 2002.

95. Cole SP, Bhardwaj G, Gerlach JH, Mackie JE, et al. Overexpression of a transporter gene in a multidrug-resistant human lung cancer cell line [see comments], Science, 258: 1650-1654, 1992.

96. Kruh GD, Gaughan KT, Godwin A, and Chan A. Expression pattern of MRP in human tissues and adult solid tumor cell lines, J Natl Cancer Inst, 87: 1256-1258, 1995. 
97. Zaman GJ, Versantvoort CH, Smit JJ, Eijdems EW, et al. Analysis of the expression of MRP, the gene for a new putative transmembrane drug transporter, in human multidrug resistant lung cancer cell lines, Cancer Res, 53: 1747-1750, 1993.

98. Flens MJ, Zaman GJ, van d, V, Izquierdo MA, et al. Tissue distribution of the multidrug resistance protein, Am J Pathol, 148: 1237-1247, 1996.

99. Deeley RG and Cole SP. Substrate recognition and transport by multidrug resistance protein 1 (ABCC1), FEBS Lett, 580: 1103-1111, 2006.

100. Chen ZS, Furukawa T, Sumizawa T, Ono K, et al. ATP-Dependent efflux of CPT11 and SN-38 by the multidrug resistance protein (MRP) and its inhibition by PAK-104P, Mol Pharmacol, 55: 921-928, 1999.

101. Allen JD, Brinkhuis RF, van Deemter L, Wijnholds J, et al. Extensive contribution of the multidrug transporters P-glycoprotein and Mrp1 to basal drug resistance, Cancer Res, 60: 5761-5766, 2000.

102. Toh S, Wada M, Uchiumi T, Inokuchi A, et al. Genomic structure of the canalicular multispecific organic anion-transporter gene (MRP2/cMOAT) and mutations in the ATP-binding-cassette region in Dubin-Johnson syndrome, Am J Hum Genet, 64: 739-746, 1999.

103. Buchler M, Konig J, Brom M, Kartenbeck J, et al. cDNA cloning of the hepatocyte canalicular isoform of the multidrug resistance protein, cMrp, reveals a novel conjugate export pump deficient in hyperbilirubinemic mutant rats, J Biol Chem, 271: 15091-15098, 1996.

104. Jedlitschky G, Leier I, Buchholz U, Hummel-Eisenbeiss J, et al. ATP-dependent transport of bilirubin glucuronides by the multidrug resistance protein MRP1 and its hepatocyte canalicular isoform MRP2, Biochem J, 327 ( Pt 1): 305-310, 1997.

105. Scheffer GL, Kool M, Heijn M, de Haas M, et al. Specific detection of multidrug resistance proteins MRP1, MRP2, MRP3, MRP5, and MDR3 P-glycoprotein with a panel of monoclonal antibodies, Cancer Res, 60: 5269-5277, 2000.

106. Koike K, Kawabe T, Tanaka T, Toh S, et al. A canalicular multispecific organic anion transporter (cMOAT) antisense cDNA enhances drug sensitivity in human hepatic cancer cells, Cancer Res, 57: 5475-5479, 1997.

107. Hooijberg JH, Broxterman HJ, Kool M, Assaraf YG, et al. Antifolate resistance mediated by the multidrug resistance proteins MRP1 and MRP2, Cancer Res, 59: 2532-2535, 1999.

108. Cui Y, Konig J, Buchholz JK, Spring H, et al. Drug resistance and ATPdependent conjugate transport mediated by the apical multidrug resistance 
protein, MRP2, permanently expressed in human and canine cells, Mol Pharmacol, 55: 929-937, 1999.

109. Chu XY, Kato Y, Niinuma K, Sudo KI, et al. Multispecific organic anion transporter is responsible for the biliary excretion of the camptothecin derivative irinotecan and its metabolites in rats, J Pharmacol Exp Ther, 281: 304-314, 1997.

110. Norris MD, Smith J, Tanabe K, Tobin P, et al. Expression of multidrug transporter MRP4/ABCC4 is a marker of poor prognosis in neuroblastoma and confers resistance to irinotecan in vitro, Mol Cancer Ther, 4: 547-553, 2005.

111. Schuetz JD, Connelly MC, Sun D, Paibir SG, et al. MRP4: A previously unidentified factor in resistance to nucleoside- based antiviral drugs, Nat Med, 5: 1048-1051, 1999.

112. Jedlitschky G, Tirschmann K, Lubenow LE, Nieuwenhuis HK, et al. The nucleotide transporter MRP4 (ABCC4) is highly expressed in human platelets and present in dense granules, indicating a role in mediator storage, Blood, 104: 3603 3610, 2004.

113. Kool M, de Haas M, Scheffer GL, Scheper RJ, et al. Analysis of expression of cMOAT (MRP2), MRP3, MRP4, and MRP5, homologues of the multidrug resistance-associated protein gene (MRP1), in human cancer cell lines, Cancer Res, 57: 3537-3547, 1997.

114. Leggas M, Adachi M, Scheffer GL, Sun D, et al. Mrp4 confers resistance to topotecan and protects the brain from chemotherapy, Mol Cell Biol, 24: 7612$7621,2004$.

115. van Aubel RA, Smeets PH, Peters JG, Bindels RJ, et al. The MRP4/ABCC4 gene encodes a novel apical organic anion transporter in human kidney proximal tubules: putative efflux pump for urinary cAMP and cGMP, J Am Soc Nephrol, 13: 595-603, 2002.

116. Zelcer N, Reid G, Wielinga P, Kuil A, et al. Steroid and bile acid conjugates are substrates of human multidrug-resistance protein (MRP) 4 (ATP-binding cassette C4), Biochem J, 371: 361-367, 2003.

117. Chen ZS, Lee K, and Kruh GD. Transport of cyclic nucleotides and estradiol 17beta-D-glucuronide by multidrug resistance protein 4 . Resistance to 6mercaptopurine and 6-thioguanine, J Biol Chem, 276: 33747-33754, 2001.

118. Reid G, Wielinga P, Zelcer N, Van DH, I, et al. The human multidrug resistance protein MRP4 functions as a prostaglandin efflux transporter and is inhibited by nonsteroidal antiinflammatory drugs, Proc Natl Acad Sci U S A, 100: 9244-9249, 2003. 
119. Chen ZS, Lee K, Walther S, Raftogianis RB, et al. Analysis of methotrexate and folate transport by multidrug resistance protein 4 (ABCC4): MRP4 is a component of the methotrexate efflux system, Cancer Res, 62: 3144-3150, 2002.

120. Tian Q, Zhang J, Tan TM, Chan E, et al. Human multidrug resistance associated protein 4 confers resistance to camptothecins, Pharm Res, 22: 1837-1853, 2005.

121. Rius M, Nies AT, Hummel-Eisenbeiss J, Jedlitschky G, et al. Cotransport of reduced glutathione with bile salts by MRP4 (ABCC4) localized to the basolateral hepatocyte membrane, Hepatology, 38: 374-384, 2003.

122. Tian Q, Zhang J, Chan SY, Tan TM, et al. Topotecan is a substrate for multidrug resistance associated protein 4, Curr Drug Metab, 7: 105-118, 2006.

123. Doyle LA, Yang W, Abruzzo LV, Krogmann T, et al. A multidrug resistance transporter from human MCF-7 breast cancer cells [published erratum appears in Proc Natl Acad Sci U S A 1999 Mar 2;96(5):2569], Proc Natl Acad Sci U S A, 95: 15665-15670, 1998.

124. Allikmets R, Schriml LM, Hutchinson A, Romano-Spica V, et al. A human placenta-specific ATP-binding cassette gene (ABCP) on chromosome 4q22 that is involved in multidrug resistance, Cancer Res, 58: 5337-5339, 1998.

125. Maliepaard M, van Gastelen MA, de Jong LA, Pluim D, et al. Overexpression of the BCRP/MXR/ABCP gene in a topotecan-selected ovarian tumor cell line, Cancer Res, 59: 4559-4563, 1999.

126. Kage K, Tsukahara S, Sugiyama T, Asada S, et al. Dominant-negative inhibition of breast cancer resistance protein as drug efflux pump through the inhibition of S-S dependent homodimerization, Int J Cancer, 97: 626-630, 2002.

127. Maliepaard M, Scheffer GL, Faneyte IF, van Gastelen MA, et al. Subcellular localization and distribution of the breast cancer resistance protein transporter in normal human tissues, Cancer Res, 61: 3458-3464, 2001.

128. Litman $\mathrm{T}$, Brangi $\mathrm{M}$, Hudson $\mathrm{E}$, Fetsch $\mathrm{P}$, et al. The multidrug-resistant phenotype associated with overexpression of the new ABC half-transporter, MXR (ABCG2), J Cell Sci, 113 ( Pt 11): 2011-2021, 2000.

129. Houghton PJ, Germain GS, Harwood FC, Schuetz JD, et al. Imatinib mesylate is a potent inhibitor of the ABCG2 (BCRP) transporter and reverses resistance to topotecan and SN-38 in vitro, Cancer Res, 64: 2333-2337, 2004.

130. Stewart CF, Leggas M, Schuetz JD, Panetta JC, et al. Gefitinib enhances the antitumor activity and oral bioavailability of irinotecan in mice, Cancer Res, 64: 7491-7499, 2004. 
131. Abe T, Koike K, Ohga T, Kubo T, et al. Chemosensitisation of spontaneous multidrug resistance by a 1,4-dihydropyridine analogue and verapamil in human glioma cell lines overexpressing MRP or MDR1, Br J Cancer, 72: 418-423, 1995.

132. Bai J, Lai L, Yeo HC, Goh BC, et al. Multidrug resistance protein 4 (MRP4/ABCC4) mediates efflux of bimane-glutathione, Int J Biochem Cell Biol, 36: 247-257, 2004.

133. Leier I, Jedlitschky G, Buchholz U, Cole SP, et al. The MRP gene encodes an ATP-dependent export pump for leukotriene $\mathrm{C} 4$ and structurally related conjugates, J Biol Chem, 269: 27807-27810, 1994.

134. Leier I, Hummel-Eisenbeiss J, Cui Y, and Keppler D. ATP-dependent paraaminohippurate transport by apical multidrug resistance protein MRP2, Kidney Int, 57: 1636-1642, 2000.

135. Chen ZS, Hopper-Borge E, Belinsky MG, Shchaveleva I, et al. Characterization of the transport properties of human multidrug resistance protein 7 (MRP7, ABCC10), Mol Pharmacol, 63: 351-358, 2003.

136. Leggas M, Panetta JC, Zhuang Y, Schuetz JD, et al. Gefitinib modulates the function of multiple ATP-binding cassette transporters in vivo, Cancer Res, 66: 4802-4807, 2006.

137. Hyafil $\mathrm{F}$, Vergely $\mathrm{C}, \mathrm{Du} \mathrm{VP}$, and Grand-Perret $\mathrm{T}$. In vitro and in vivo reversal of multidrug resistance by GF120918, an acridonecarboxamide derivative, Cancer Res, 53: 4595-4602, 1993.

138. Allen JD, van LA, Lakhai JM, van d, V, et al. Potent and specific inhibition of the breast cancer resistance protein multidrug transporter in vitro and in mouse intestine by a novel analogue of fumitremorgin C, Mol Cancer Ther, 1: 417-425, 2002.

139. Maliepaard M, van Gastelen MA, Tohgo A, Hausheer FH, et al. Circumvention of breast cancer resistance protein (BCRP)-mediated resistance to camptothecins in vitro using non-substrate drugs or the BCRP inhibitor GF120918, Clin Cancer Res, 7: 935-941, 2001.

140. Allen JD, Brinkhuis RF, Wijnholds J, and Schinkel AH. The mouse Bcrp1/Mxr/Abcp gene: amplification and overexpression in cell lines selected for resistance to topotecan, mitoxantrone, or doxorubicin, Cancer Res, 59: 42374241, 1999.

141. Decleves X, Fajac A, Lehmann-Che J, Tardy M, et al. Molecular and functional MDR1-Pgp and MRPs expression in human glioblastoma multiforme cell lines, Int J Cancer, 98: 173-180, 2002. 
142. Fire A, Xu S, Montgomery MK, Kostas SA, et al. Potent and specific genetic interference by double-stranded RNA in Caenorhabditis elegans, Nature, 391: 806-811, 1998.

143. Agrawal N, Dasaradhi PV, Mohmmed A, Malhotra P, et al. RNA interference: biology, mechanism, and applications, Microbiol Mol Biol Rev, 67: 657-685, 2003.

144. Montgomery MK and Fire A. Double-stranded RNA as a mediator in sequencespecific genetic silencing and co-suppression, Trends Genet, 14: 255-258, 1998.

145. Dillin A. The specifics of small interfering RNA specificity, Proc Natl Acad Sci U S A, 100: 6289-6291, 2003.

146. Zamore PD, Tuschl T, Sharp PA, and Bartel DP. RNAi: double-stranded RNA directs the ATP-dependent cleavage of mRNA at 21 to 23 nucleotide intervals, Cell, 101: 25-33, 2000.

147. Ngo H, Tschudi C, Gull K, and Ullu E. Double-stranded RNA induces mRNA degradation in Trypanosoma brucei, Proc Natl Acad Sci U S A, 95: 14687-14692, 1998.

148. Montgomery MK, Xu S, and Fire A. RNA as a target of double-stranded RNAmediated genetic interference in Caenorhabditis elegans, Proc Natl Acad Sci U S A, 95: 15502-15507, 1998.

149. Mittal V. Improving the efficiency of RNA interference in mammals, Nat Rev Genet, 5: 355-365, 2004.

150. Semizarov D, Frost L, Sarthy A, Kroeger P, et al. Specificity of short interfering RNA determined through gene expression signatures, Proc Natl Acad Sci U S A, 100: 6347-6352, 2003.

151. Brummelkamp TR, Bernards R, and Agami R. A system for stable expression of short interfering RNAs in mammalian cells, Science, 296: 550-553, 2002.

152. Castel V and Canete A. A comparison of current neuroblastoma chemotherapeutics, Expert Opin Pharmacother, 5: 71-80, 2004.

153. Houghton PJ, Cheshire PJ, Hallman JD, Lutz L, et al. Efficacy of topoisomerase I inhibitors, topotecan and irinotecan, administered at low dose levels in protracted schedules to mice bearing xenografts of human tumors, Cancer Chemother Pharmacol, 36: 393-403, 1995.

154. Zamboni WC, Houghton PJ, Hulstein JL, Kirstein M, et al. Relationship between tumor extracellular fluid exposure to topotecan and tumor response in human neuroblastoma xenograft and cell lines, Cancer Chemother Pharmacol, 43: 269276, 1999. 
155. Zamboni WC, Stewart CF, Thompson J, Santana VM, et al. Relationship between topotecan systemic exposure and tumor response in human neuroblastoma xenografts, Journal of National Cancer Institute, 90: 505-511, 1998.

156. Norris MD, Bordow SB, Marshall GM, Haber PS, et al. Expression of the gene for multidrug-resistance-associated protein and outcome in patients with neuroblastoma, N Engl J Med, 334: 231-238, 1996.

157. Schellens JH, Maliepaard M, Scheper RJ, Scheffer GL, et al. Transport of topoisomerase I inhibitors by the breast cancer resistance protein. Potential clinical implications, Ann N Y Acad Sci, 922: 188-194, 2000.

158. Maliepaard M, Scheffer GL, Faneyte IF, van Gastelen MA, et al. Subcellular localization and distribution of the breast cancer resistance protein transporter in normal human tissues, Cancer Res, 61: 3458-3464, 2001.

159. Amarzguioui M, Rossi JJ, and Kim D. Approaches for chemically synthesized siRNA and vector-mediated RNAi, FEBS Lett, 579: 5974-5981, 2005.

160. Persons DA, Allay JA, Allay ER, Smeyne RJ, et al. Retroviral-mediated transfer of the green fluorescent protein gene into murine hematopoietic cells facilitates scoring and selection of transduced progenitors in vitro and identification of genetically modified cells in vivo, Blood, 90: 1777-1786, 1997.

161. Adachi M, Sampath J, Lan LB, Sun D, et al. Expression of MRP4 confers resistance to ganciclovir and compromises bystander cell killing, J Biol Chem, 277: 38998-39004, 2002.

162. Wunderlich K, Zimmerman C, Gutmann H, Teuchner B, et al. Vasospastic persons exhibit differential expression of ABC-transport proteins, Mol Vis, 9: 756-761, 2003.

163. Assem M, Schuetz EG, Leggas M, Sun D, et al. Interactions between hepatic Mrp4 and Sult2a as revealed by the constitutive androstane receptor and Mrp4 knockout mice, J Biol Chem, 279: 22250-22257, 2004.

164. Zamber CP, Lamba JK, Yasuda K, Farnum J, et al. Natural allelic variants of breast cancer resistance protein (BCRP) and their relationship to BCRP expression in human intestine, Pharmacogenetics, 13: 19-28, 2003.

165. Murray LJ, Bruno E, Uchida N, Hoffman R, et al. CD109 is expressed on a subpopulation of $\mathrm{CD} 34+$ cells enriched in hematopoietic stem and progenitor cells, Exp Hematol, 27: 1282-1294, 1999.

166. McKenzie PP, McPake CR, Ashford AA, Vanin EF, et al. MDM2 does not influence p53-mediated sensitivity to DNA-damaging drugs, Mol Cancer Ther, 1: 1097-1104, 2002. 
167. McPake CR, Tillman DM, Poquette CA, George EO, et al. Bax is an important determinant of chemosensitivity in pediatric tumor cell lines independent of Bcl-2 expression and p53 status, Oncol Res, 10: 235-244, 1998.

168. Middlemas DS, Stewart CF, Kirstein MN, Poquette C, et al. Biochemical correlates of temozolomide sensitivity in pediatric solid tumor xenograft models, Clin Cancer Res, 6: 998-1007, 2000.

169. Friedenberg WR, Rue M, Blood EA, Dalton WS, et al. Phase III study of PSC833 (valspodar) in combination with vincristine, doxorubicin, and dexamethasone (valspodar/VAD) versus VAD alone in patients with recurring or refractory multiple myeloma (E1A95): a trial of the Eastern Cooperative Oncology Group, Cancer, 106: 830-838, 2006.

170. Nobili S, Landini I, Giglioni B, and Mini E. Pharmacological strategies for overcoming multidrug resistance, Curr Drug Targets, 7: 861-879, 2006.

171. Bates SE, Bakke S, Kang M, Robey RW, et al. A phase I/II study of infusional vinblastine with the P-glycoprotein antagonist valspodar (PSC 833) in renal cell carcinoma, Clin Cancer Res, 10: 4724-4733, 2004.

172. Turner PK, Johnston B, Wingo S, Schuetz JD, et al. MRP4 and P-glycoprotein (PgP) expression associated with topotecan (TPT) sensitivity in neuroblastoma (NB) cell lines, Proc Am Assoc Cancer Res, 44: 2003.

173. Graham C, Tucker C, Creech J, Favours E, et al. Evaluation of the antitumor efficacy, pharmacokinetics, and pharmacodynamics of the histone deacetylase inhibitor depsipeptide in childhood cancer models in vivo, Clin Cancer Res, 12: 223-234, 2006.

174. Thompson J, Guichard SM, Cheshire PJ, Richmond LB, et al. Development, characterization and therapy of a disseminated model of childhood neuroblastoma in SCID mice, Cancer Chemother Pharmacol, 47: 211-221, 2001.

175. Hausser HJ and Brenner RE. Phenotypic instability of Saos-2 cells in long-term culture, Biochem Biophys Res Commun, 333: 216-222, 2005.

176. Maitra A, Arking DE, Shivapurkar N, Ikeda M, et al. Genomic alterations in cultured human embryonic stem cells, Nat Genet, 37: 1099-1103, 2005.

177. Fiorani P and Bjornsti MA. Mechanisms of DNA topoisomerase I-induced cell killing in the yeast Saccharomyces cerevisiae, Ann N Y Acad Sci, 922: 65-75, 2000 .

178. Kuo CC, Liu JF, and Chang JY. DNA repair enzyme, O6-methylguanine DNA methyltransferase, modulates cytotoxicity of camptothecin-derived topoisomerase I inhibitors, J Pharmacol Exp Ther, 316: 946-954, 2006. 
179. Hawley RG, Lieu FH, Fong AZ, and Hawley TS. Versatile retroviral vectors for potential use in gene therapy, Gene Ther, 1: 136-138, 1994.

180. Krishnamurthy P, Ross DD, Nakanishi T, Bailey-Dell K, et al. The stem cell marker Bcrp/ABCG2 enhances hypoxic cell survival through interactions with heme, J Biol Chem, 279: 24218-24225, 2004.

181. De PK, Vandesompele J, Heimann P, Yigit N, et al. Human fetal neuroblast and neuroblastoma transcriptome analysis confirms neuroblast origin and highlights neuroblastoma candidate genes, Genome Biol, 7: R84-2006.

182. Weiss WA, Aldape K, Mohapatra G, Feuerstein BG, et al. Targeted expression of MYCN causes neuroblastoma in transgenic mice, EMBO J, 16: 2985-2995, 1997.

183. Aigner A. Delivery Systems for the Direct Application of siRNAs to Induce RNA Interference (RNAi) In Vivo, J Biomed Biotechnol, 2006: 71659-2006.

184. Simon T, Langler A, Berthold F, Klingebiel T, et al. Topotecan and etoposide in the treatment of relapsed high-risk neuroblastoma: results of a phase 2 trial, $\mathrm{J}$ Pediatr Hematol Oncol, 29: 101-106, 2007.

185. Kushner BH, Kramer K, Modak S, and Cheung NK. Camptothecin analogs (irinotecan or topotecan) plus high-dose cyclophosphamide as preparative regimens for antibody-based immunotherapy in resistant neuroblastoma, Clin Cancer Res, 10: 84-87, 2004. 


\section{Vita}

Patricia Kellie Turner was born in Memphis, Tennessee on September 22, 1976. She attended Dogwood Elementary School and graduated from Houston High School in May, 1994. The following September she matriculated at Boston College and in May, 1998 received the degree Bachelor of Science in Biochemistry. In August, 1998 she entered the Pharm.D./ Ph.D. program at the University of Tennessee Health Science Center and received the Pharm.D. degree in June, 2002. Upon successfully defending her dissertation in May 2007, she plans to begin a Cancer Research UK funded postdoctoral fellowship at the Northern Institute for Cancer Research at Newcastle University. Her postdoctoral research will be in the field of breast cancer pharmacokinetics, pharmacogenetics, pharmacodynamics, and population modeling. 